\title{
Machine learning algorithms for identification of low signal-to-noise ratio radar signals
}

\author{
Anne Young, B.Sc. \\ A thesis submitted to the \\ Faculty of Graduate and Postdoctoral Affairs \\ in partial fulfillment of the requirements for the degree of
}

Master of Applied Science in Electrical Engineering

Ottawa-Carleton Institute for Electrical and Computer Engineering

Department of Systems and Computer Engineering

Carleton University

Ottawa, Ontario

August, 2021

(C) 2021

Anne Young 


\begin{abstract}
Areas such as spectrum monitoring require identification of known and unknown radar transmitters to identify known and rogue users. Often such identification needs to done under conditions where the signal-to-noise ratio is low. This thesis proposes an approach to determine the unknown radar chirp parameters of a linear frequency modulated (LFM) radar waveform, assuming that the unknown parameters come from a given set of known chirp parameters. A concatenated output of matched filters corresponding to the known set of chirp parameters is presented to four well-known machine learning architectures, namely decision tree (DT), random forest (RnF), naïve Bayes (NB) and support vector machine (SVM). Realistic radar parameters for airborne, marine and weather radars were used in the simulations. The robustness of the classifiers to parameter mismatch and truncation of the radar pulse were also studied. DT outperformed the other classifiers except for the truncated pulse case (where NB and SVM performed better). RnF did not perform acceptably.
\end{abstract}




\section{Acknowledgments}

First, I would like to extend my heartfelt gratitude to my supervisors, Prof. Sreeraman Rajan and Dr. Bhashyam Balaji, for their unparalleled guidance, mentorship, and scientific advice. They have inspired me to learn and grow as a researcher and as a person. Secondly, I would like to thank my research group colleague, David Luong, for his invaluable technical aid, his feedback on my work, and going above and beyond to help me finish. I could not have completed this thesis without Prof. Rajan, Dr. Balaji and David Luong in these strange times. I would also like to acknowledge Prof. Mae Seto and Dr. Chuck Livingstone for their support, belief in me, and the impetus to undertake this journey.

Finally, I would like to thank my family and friends for their love, support and encouragement. I have truly been blessed to have my parents, Helena and Dick Wagenaar, there for me throughout my life. My sons, Luke and Adam, mean the world to me. 


\section{Table of Contents}

Abstract $\quad$ ii

Acknowledgments $\quad$ iii

Table of Contents $\quad$ iv

List of Tables $\quad$ vi

List of Figures $\quad$ vii

Nomenclature $\quad$ xi

1 Introduction 1

1.1 Motivation . . . . . . . . . . . . . . . . . . 1

1.2 Scope of the Research . . . . . . . . . . . . . . . . . . 3

1.3 Contributions of the thesis . . . . . . . . . . . . . 4

1.4 Thesis Outline . . . . . . . . . . . . . . . . 5

2 Literature review $\quad 7$

2.1 Summary .......................... 15

3 Radar Signal Processing and Machine Learning $\quad 16$

3.1 Introduction . . . . . . . . . . . . . . . . . . . . . 16

3.2 Fundamentals of Radars and Radar Intercept Receivers . . . . . . . . 16

3.3 Linear frequency modulation (LFM) modulation . . . . . . . . . . . . 18

3.4 Wigner-Ville Distribution . . . . . . . . . . . . . . . 19

3.5 Machine Learning algorithms and performance metrics . . . . . . . . 20

3.5.1 Support Vector Machine (SVM) Classifier . . . . . . . . . . . 21

3.5.2 Naive Bayes (NB) Classifier . . . . . . . . . . . . . 23 
3.5.3 Decision Tree (DT) Classifier . . . . . . . . . . . . . . 24

3.5.4 Random Forest (RnF) Classifier . . . . . . . . . . . . 25

3.6 Machine Learning Metrics . . . . . . . . . . . . . . . . . 25

4 Some Common Radars $\quad 29$

4.1 Introduction . . . . . . . . . . . . . . . . . 29

4.2 Weather radars . . . . . . . . . . . . . . . 29

4.3 Marine radars . . . . . . . . . . . . . . . . . . 31

4.4 Airborne radars . . . . . . . . . . . . . . . . . . 36

4.5 Summary . . . . . . . . . . . . . . . . . . 40

5 Results $\quad 43$

5.1 Introduction . . . . . . . . . . . . . . . . . . 43

5.2 Inadequacy of the Wigner-Ville Transform . . . . . . . . . . . . . 43

5.3 Proposed approach . . . . . . . . . . . . . . . . 43

5.4 Classifiers Evaluated in This Thesis . . . . . . . . . . . . . . 47

5.4.1 Details of Simulations . . . . . . . . . . . . . . . 49

5.5 Results of Simulations . . . . . . . . . . . . . . 50

5.5.1 Problem 1: LFM signals with pre-selected chirp rates . . . . . 50

5.5.2 Problem 2: Airborne radar realistic chirp results . . . . . . . . 51

5.5.3 Problem 3: Weather radar realistic chirp results . . . . . . . . 58

5.5.4 Problem 4: Marine radar realistic chirp results . . . . . . . . 62

5.5.5 Problem 5: All realistic radar chirp rates results . . . . . . . 69

5.5.6 Problem 6: Robustness of the classifiers against windowing imperfections ...................... 69

5.5.7 Problem 7: Robustness of the classifier against perturbation in chirp parameters. . . . . . . . . . . . . 77

5.6 Summary . . . . . . . . . . . . . . . . . . . . 77

6 Conclusions and Way Forward $\quad 80$

6.1 Limitations . . . . . . . . . . . . . . . . . . . . . . 82

6.2 Future work . . . . . . . . . . . . . . . . . . . 82

List of References $\quad 83$ 


\section{List of Tables}

2 Nexrad WSR Weather Radar Parameters . . . . . . . . . . . . 32

3 Marine radar frequency band allocations [1] . . . . . . . . . . . 33

4 Typical characteristics of IMO category radio navigation radars. . . . 33

5 Typical characteristics of river class radio navigation radars. . . . . . 34

6 Typical characteristics of pleasure-craft class radio navigation radars. 35

7 Furono FR7111 Marine Radar Parameters . . . . . . . . . . . . 37

8 Sample marine radar chirp rates. . . . . . . . . . . . . . . 38

9 Air radar pulse transmission schemes by function. . . . . . . . . . 38

10 ASR-9 airborne radar parameters. . . . . . . . . . . . . . . . 40

11 Sample airborne radar chirp rates. . . . . . . . . . . . . . . . . . 41

12 Summary of realistic chirp rates for airborne, weather and marine radars. 42

13 Radar types and signal parameters for a set of radars that are unknown to the bank of matched filters. . . . . . . . . . . . . . . 51 


\section{List of Figures}

1 Support Vector Machine Hyperplane [1] . . . . . . . . . . . . . . . 22

2 WSR 88D Weather Radar scanning pattern . . . . . . . . 30

3 Wigner-Ville transform of an LFM signal. . . . . . . . . . . . 44

4 Wigner-Ville transform of a noisy LFM signal at a SNR of $0 \mathrm{~dB}$. . . 44

5 Wigner-Ville transform of an LFM signal at a SNR of $-10 \mathrm{~dB}$. . . . 45

6 Wigner-Ville transform of a noisy LFM signal at a SNR of $-20 \mathrm{~dB} . \quad$. 45

7 Example of a feature vector obtained by concatenating all the matched filter outputs of an ideal chirp. . . . . . . . . . . . . . . . 47

8 Concatenated match filter approach applied to LFM signal at $-10 \mathrm{~dB}$. No clear peak is evident making it difficult to accurately classify the chirp rate. . . . . . . . . . . . . . . . . . . 48

9 Confusion matrix for a NB classifier at $-20 \mathrm{~dB}$ SNR (Problem 1). . . 52

10 Confusion matrix for a SVM classifier for $-20 \mathrm{~dB}$ SNR (Problem 1). . 52

11 Confusion matrix for a DT classifier of realistic airborne chirp rates at SNRs from $10 \mathrm{~dB}$ (top left), $0 \mathrm{~dB}$ (top mid), $-5 \mathrm{~dB}$ (top right), -10 $\mathrm{dB}$ (bottom left), $-15 \mathrm{~dB}$ (bottom mid) and $-20 \mathrm{~dB}$ (bottom right).

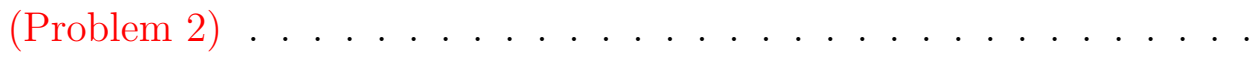

12 Confusion matrix for a NB classifier of realistic airborne radar chirp rates at SNR $10 \mathrm{~dB}$ (top left), $0 \mathrm{~dB}$ (top mid), $-5 \mathrm{~dB}$ (top right), -10 $\mathrm{dB}$ (bottom left), $-15 \mathrm{~dB}$ (bottom mid) and $-20 \mathrm{~dB}$ (bottom right).

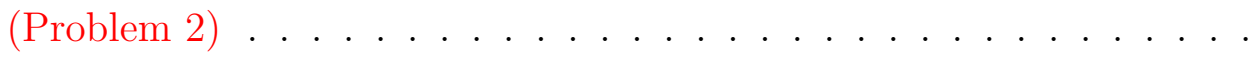

13 Confusion matrix for a $\mathrm{RnF}$ classifier of realistic airborne radar chirp rates at SNR $10 \mathrm{~dB}$ (top left), $0 \mathrm{~dB}$ (top mid), $-5 \mathrm{~dB}$ (top right), -10 $\mathrm{dB}$ (bottom left), $-15 \mathrm{~dB}$ (bottom mid) and $-20 \mathrm{~dB}$ (bottom right).

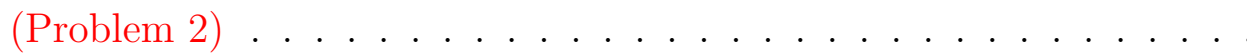


14 Confusion matrix for a SVM classifier of realistic airborne chirp rates at SNR $10 \mathrm{~dB}$ (top left), $0 \mathrm{~dB}$ (top mid), $-5 \mathrm{~dB}$ (top right), -10 dB (bottom left), $-15 \mathrm{~dB}$ (bottom mid) and $-20 \mathrm{~dB}$ (bottom right). (Problem 2) . 56

15 Accuracy versus SNR level - Airborne radar chirp rates. (Problem 2) 57

16 F1 score versus SNR level - Airborne radar chirp rates. (Problem 2) . 57

17 Confusion matrix for a DT classifier of realistic weather radar chirp rates at SNRs $10 \mathrm{~dB}$ (top left), $0 \mathrm{~dB}$ (top mid), -5 dB (top right), -10 $\mathrm{dB}$ (bottom left), $-15 \mathrm{~dB}$ (bottom mid) and $-20 \mathrm{~dB}$ (bottom right). (Problem 3) . . . . . . . . . . . . . . . . 58

18 Confusion matrix for a NB classifier of realistic weather radar chirp rates at SNR $10 \mathrm{~dB}$ (top left), $0 \mathrm{~dB}$ (top mid), $-5 \mathrm{~dB}$ (top right), -10 $\mathrm{dB}$ (bottom left), $-15 \mathrm{~dB}$ (bottom mid) and $-20 \mathrm{~dB}$ (bottom right). (Problem 3) . . . . . . . . . . . . . . . .

19 Confusion matrix for a RnF classifier of realistic weather chirp rates at SNR $10 \mathrm{~dB}$ (top left), $0 \mathrm{~dB}$ (top mid), -5 dB (top right), -10 dB (bottom left), $-15 \mathrm{~dB}$ (bottom mid) and $-20 \mathrm{~dB}$ (bottom right). (Problem 3) .

20 Confusion matrix for a SVM classifier of realistic airborne radar chirp rates at SNR $10 \mathrm{~dB}$ (top left), $0 \mathrm{~dB}$ (top mid), -5 dB (top right), -10 $\mathrm{dB}$ (bottom left), $-15 \mathrm{~dB}$ (bottom mid) and $-20 \mathrm{~dB}$ (bottom right). (Problem 3) . . . . . . . . . . . . . . . . . 6 61

21 Accuracy versus SNR level - Weather radar chirp rates. (Problem 3) . 62

22 F1 score versus SNR level - Weather radar chirp rates. (Problem 3) . 63

23 Confusion matrix for a DT classifier of realistic marine radar chirp rates at SNR $10 \mathrm{~dB}$ (top left), $0 \mathrm{~dB}$ (top mid), -5 dB (top right), -10 $\mathrm{dB}$ (bottom left), $-15 \mathrm{~dB}$ (bottom mid) and $-20 \mathrm{~dB}$ (bottom right). (Problem 4) . . . . . . . . . . . . . . . . . .

24 Confusion matrix for a NB classifier of realistic marine radar chirp rates at $\mathrm{SNR} 10 \mathrm{~dB}$ (top left), $0 \mathrm{~dB}$ (top mid), $-5 \mathrm{~dB}$ (top right), -10 $\mathrm{dB}$ (bottom left), $-15 \mathrm{~dB}$ (bottom mid) and $-20 \mathrm{~dB}$ (bottom right). (Problem 4) . . . . . . . . . . . . . . . .

25 Confusion matrix for a $\mathrm{RnF}$ classifier of realistic marine radar chirp rates at $\mathrm{SNR} 10 \mathrm{~dB}$ (top left), $0 \mathrm{~dB}$ (top mid), $-5 \mathrm{~dB}$ (top right), -10 $\mathrm{dB}$ (bottom left), $-15 \mathrm{~dB}$ (bottom mid) and $-20 \mathrm{~dB}$ (bottom right). (Problem 4) . . . . . . . . . . . . . . . . . 
26 Confusion matrix for a SVM classifier of realistic marine radar chirp rates at SNR $10 \mathrm{~dB}$ (top left), $0 \mathrm{~dB}$ (top mid) , -5 dB (top right), -10 $\mathrm{dB}$ (bottom left), $-15 \mathrm{~dB}$ (bottom mid) and $-20 \mathrm{~dB}$ (bottom right). (Problem 4) .......................... 67

27 Accuracy versus SNR level - Marine radar chirp rates. (Problem 4) . 68

28 F1 score versus SNR level - Marine radar chirp rates. (Problem 4) . . 68

29 Confusion matrix for a DT classifier of all realistic radar chirp rates at SNR $10 \mathrm{~dB}$ (top left), $0 \mathrm{~dB}$ (top mid), -5 dB (top right), -10 dB (bottom left), $-15 \mathrm{~dB}$ (bottom mid) and $-20 \mathrm{~dB}$ (bottom right). (Problem 5) .

30 Confusion matrix for a NB classifier of all realistic radar chirp rates at SNR $10 \mathrm{~dB}$ (top left), $0 \mathrm{~dB}$ (top mid), $-5 \mathrm{~dB}$ (top right), -10 dB (bottom left), $-15 \mathrm{~dB}$ (bottom mid) and $-20 \mathrm{~dB}$ (bottom right). (Problem 5)

31 Confusion matrix for a SVM classifier of all realistic radar chirp rates at SNR $10 \mathrm{~dB}$ (top left), $0 \mathrm{~dB}$ (top mid), -5 dB (top right), -10 dB (bottom left), $-15 \mathrm{~dB}$ (bottom mid) and $-20 \mathrm{~dB}$ (bottom right). (Problem 5) 71

32 Accuracy versus SNR level - All radar chirp rates. (Problem 5) . . . . 72

33 F1 score versus SNR level - All radar chirp rates. (Problem 5) . . . . 72

34 Truncated chirp of an LFM signal. (Problem 6) . . . . . . . . . . . 73

35 Confusion matrix for a NB classifier of realistic truncated chirp rates at SNR $10 \mathrm{~dB}$ (top left), $0 \mathrm{~dB}$ (top mid), -5 dB (top right), -10 dB (bottom left), $-15 \mathrm{~dB}$ (bottom mid) and $-20 \mathrm{~dB}$ (bottom right). (Problem 6) .

36 Confusion matrix for a SVM classifier of realistic chirp rates at SNR $10 \mathrm{~dB}$ (top left), $0 \mathrm{~dB}$ (top mid), $-5 \mathrm{~dB}$ (top right), $-10 \mathrm{~dB}$ (bottom left), $-15 \mathrm{~dB}$ (bottom mid) and $-20 \mathrm{~dB}$ (bottom right). (Problem 6) .

37 Accuracy versus SNR level - All radar truncated chirp rates. (Problem

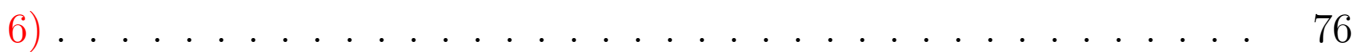

38 F1 score versus SNR level - All radar truncated chirp rates. (Problem 6) 76

39 Confusion matrix for classifying signals from unknown radars at a SNR of $10 \mathrm{~dB}$ using a DT classifier. (Problem 7) . . . . . . . . . . . . 78

40 Confusion matrix for classifying signals from unknown radars at a SNR of $0 \mathrm{~dB}$ using a DT classifier. (Problem 7) . . . . . . . . . . . . 78

41 Confusion matrix for classifying signals from unknown radars at a SNR of $-5 \mathrm{~dB}$ using a DT classifier. (Problem 7) . . . . . . . . . . . 78 
42 Confusion matrix for classifying signals from unknown radars at a SNR of $-10 \mathrm{~dB}$ using a DT classifier. (Problem 7) . . . . . . . . 79

43 Confusion matrix for classifying signals from unknown radars at a SNR of $-15 \mathrm{~dB}$ using a DT classifier. (Problem 7) . . . . . . . . . 79

44 Confusion matrix for classifying signals from unknown radars at a SNR of $-20 \mathrm{~dB}$ using a DT classifier. (Problem 7) . . . . . . . . 79 


\section{Nomenclature}

\begin{tabular}{cc}
\hline Acc & Accuracy \\
Ae & Effective Area \\
AESA & Advanced Electronically Scanned Array \\
ANN & Artificial Neural Network \\
ARSR & Air Route Surveillance Radars \\
ASL & Above Sea Level \\
ASR & Airport Surveillance Radars \\
AUC & Area Under the Curve \\
Az & Azimuth \\
BPSK & Barker Phase Shift Keying \\
BW & Bandwidth \\
CCQP & Convex Constrained Quadratic Programming \\
CFAR & Constant False Alarm Rate \\
CM & Confusion Matrix \\
cm & Centimetre \\
CNN & Decibel (zero weighted) \\
CW & Continuous Wave \\
dB & Decibel \\
dBz & Convolutional Neural Network \\
& Con
\end{tabular}




\begin{tabular}{|c|c|}
\hline DNN & Deep Neural Network \\
\hline DT & Decision Tree \\
\hline EL & Elevation \\
\hline ER & Error Rate \\
\hline $\mathrm{fd}$ & Frequency \\
\hline FFT & Fast Fourier Transform \\
\hline FM & Frequency Modulation \\
\hline FMCW & Frequency Modulated Continuous Wave \\
\hline FN & False Negative \\
\hline $\mathrm{FP}$ & False Positive \\
\hline FRFT & Fractional Fourier Transform \\
\hline FSK & Frequency Shift Keying \\
\hline Gt,r & Antenna Gain, Receive and Transmit \\
\hline $\mathrm{GHz}$ & Giga Hertz \\
\hline $\mathrm{H}$ & Horizontal \\
\hline $\mathrm{HT}$ & Hough Transform \\
\hline $\mathrm{Hz}$ & Hertz \\
\hline IPM & Intra-pulse Modulation \\
\hline $\mathrm{k}$ & Boltzmann's Constant \\
\hline $\mathrm{Km}$ & Kilometre \\
\hline $\mathrm{kW}$ & Kilo Watt \\
\hline $\mathrm{IF}$ & Intermediate Frequency \\
\hline IMO & International Maritime Organisation \\
\hline IMOP & Intra-modulation on Pulse \\
\hline
\end{tabular}




\begin{tabular}{|c|c|}
\hline ITU & International Telecommunications Union \\
\hline LFM & Linear Frequency Modulation \\
\hline FMCW & Linear Frequency Modulation Continuous Wave \\
\hline LPI & Low Probability of Intercept \\
\hline LS & Least Squares \\
\hline LSTM & Long Short-term Memory \\
\hline $\mathrm{MF}$ & Matched Filter \\
\hline $\mathrm{MHz}$ & Mega Hertz \\
\hline ML & Machine Learning \\
\hline MLE & Maximum Likelihood Estimation \\
\hline $\mathrm{ms}$ & Millisecond \\
\hline MSE & Mean Squared Error \\
\hline MW & Mega Watt \\
\hline $\mathrm{N}$ & Negative \\
\hline $\mathrm{NN}$ & Neural Networks \\
\hline No & Receiver Noise \\
\hline NB & Naïve Bayes \\
\hline NLFM & Non-linear Frequency Modulation \\
\hline $\mathrm{nm}$ & Nautical Mile \\
\hline $\mathrm{NN}$ & Neural Network \\
\hline NR & No Radar \\
\hline $\mathrm{P}$ & Positive \\
\hline $\mathrm{PA}$ & Pulse Amplitude \\
\hline PAR & Precision Approach Radar \\
\hline
\end{tabular}


PC

PD

$\mathrm{Pd}$

$\operatorname{Pr}$

PRC

Prec

PRF

PRI

PRR

$\mathrm{Pt}$

PW

QPSK

$\mathrm{R}$

RCS

RF

$\mathrm{RnF}$

RMSE

rpm

SNR

STFT

SVM

TF

To

TN
Pulse Carrier

Pulse Distribution

Power Density

Received Power

Precision Recall Curve

Precision

Pulse Repetition Frequency

Pulse Repetition Interval

Pulse Repetition Rate

Transmitted Power

Pulse Width

Quadrature Phase Shift Keying

Range

Radar Cross Section

Radio Frequency

Random Forrest

Root Mean Squared Error

Revolutions per Minute

Signal to Noise ratio

Short-Time Frequency Transform

Support Vector Machine

Time Frequency

Standard Environmental Temperature

True Negative 
TP

V

W

WVD

WH

WVH
True Positive

Vertical

Watt

Wigner-Ville Distribution

Wigner-Hough

Wigner-Ville Hough 


\section{Chapter 1}

\section{Introduction}

\subsection{Motivation}

In radio frequency $(\mathrm{RF})$ spectrum monitoring applications, it is of importance to determine whether radar transmitters in a given location are operating legitimately. These environments are abundant with different classes of radar waveforms (e.g. waveforms corresponding to marine, airborne, weather, and automotive radars). Harbours and airports are instances of particularly congested electromagnetic spectrum environments [2]. Unregulated radars that do not have licenses to operate in the frequency band for the appropriate geographical area could interfere with legally regulated radar emitters in these environments, potentially impacting the safe and secure operation of harbours or airports and creating serious economic damage. Pirates or illegal fishing vessels, for example, may use illicit radar transmitters, and suppressing their activities is a clear public safety goal. Therefore, the ability to determine the presence/absence of a radar waveform and (if present) determine its parameters and classify its type, is particularly important for spectrum monitoring authorities in environments such as airports and harbours. Unfortunately, this problem is made difficult by the fact that unlicensed radars tend to be low probability of intercept (LPI) radars: they are designed to be difficult to detect. They may, for instance, transmit only during short periods of time. For these reasons, a technique to classify the radar signals in a certain area would be of great utility to spectrum monitoring authorities, especially in low-Signal-to-Noise Ratio (SNR) scenarios such as at long radar ranges.

A waveform commonly employed in commercial radars is the linear frequency modulated (LFM) waveform. It is therefore of particular interest to estimate the parameters of LFM waveforms. This thesis proposes an approach to determine the 
unknown radar chirp parameters of a LFM radar waveform, assuming that the unknown parameters come from a given set of known chirp parameters. Unlike many competing techniques, the approach studied here works at low signal-to-noise ratios (SNRs). The proposed identification of the chirp parameters in this thesis is done on a single-pulse basis without the need to process multiple pulses. This approach enables the rapid identification of emitters when intercepted passively by an intercepting radar receiver.

Often, one may not have the luxury of collecting a lot of pulses to enhance detection performance, particularly in the low-SNR scenario. Under these circumstances, signal processing and decision making has to be conducted with very a limited amount of data. This problem has not drawn wide attention in the literature, which has hitherto typically focused on older radars (such as mechanically scanned array radars) for which it was a safe assumption that the same waveform was used for several pulses. Currently, most receivers operate using a threshold detector, and if detection is declared then the signal parameters are estimated. These detection techniques often use multiple pulses and hence require a high probability of intercept for the individual pulses [3]. The signal parameter estimation step typically relies on time-frequency analysis techniques such as the Wigner-Ville transform [4] or the Hough transform, which fail at low SNRs (as shown later in this thesis). It may be argued that one could boost the SNR by integrating multiple pulses, but this leads to many practical difficulties. In any case, the integration process may require more time than may be available to classify an unknown emitter.

Modern radars are typically advanced electronically scanned array (AESA) radars. AESA radars can be programmed in such a way that each pulse can have different waveforms corresponding to different radar modes, subject only to hardware constraints. In short, they offer the flexibility of selecting waveforms on a pulse-by-pulse basis. It is more challenging to classify radar signals when the waveform parameters vary pulse by pulse. Hence, the need for recognition of waveform parameters on a pulse-by-pulse basis. The research in this thesis is intended to explore the possibilities available for the identification of emitters based on noisy single pulse data.

Traditionally, most radar signal processing at the receiver side begins with matched filtering. This motivates the use of matched filter outputs for classification purposes, a use which is surprisingly rare in the literature. In this thesis, supervised 
machine learning algorithms are used to identify pulsed LFM radars using the outputs of a bank of matched filters. Because the approach in this thesis is totally novel and has never been studied before, the analysis represents only a preliminary application of the combination of matched filtering and machine learning. In this work, only raw matched filter outputs are considered as inputs to the classifiers; there was no use of feature selection or extraction. In addition, we have not used any of the state-of-the-art machine learning techniques based on neural networks that have had tremendous success in solving many real world problems. However, the investigations carried out in this thesis gives a lower bound on the possible performance benefits of the proposed approach.

\subsection{Scope of the Research}

The problem solved in this thesis is as follows: given a LFM radar pulse whose defining parameters (carrier frequency and chirp rate) are taken from a known set of parameters, and which may be contaminated with additive white Gaussian noise, determine the parameters corresponding to the given LFM pulse.

It is assumed that the intercept receiver is tuned properly to receive the radar signal and that the radar emitters and the intercept receivers are both stationary. Doppler effects are not considered as they will not impact the performance as only single pulse LFM signals are considered in this work. It is further assumed that a given recording contains at most one radar pulse; the problem of distinguishing multiple LFM pulses within a single recording is left for future work. Likewise, the only type of interference is additive white Gaussian noise; any other type of interference (e.g. channel impairments or clutter) is left for future work.

In order to solve this problem, this thesis adopts a technique based on matched filtering (MF). The motivation for MF-based algorithms is that MF optimizes the SNR. The match filter output is then fed into a machine learning algorithm to classify the LFM signal. Thus, in this work, no further transformations for extraction of features are considered. A bank of matched filters corresponding to the chirp parameters from the known set of parameters is itself considered as the feature vector and is used as input to the classifiers.

The proposed machine learning methodology will be tested using single LFM pulses with a set of predetermined chirp rates. The failure to identify the chirp 
rate based on the traditional Wigner-Ville transform is demonstrated in this work, thus motivating the need for the proposed method. Two sets of LFM parameters are used: a small and simple set of unknown radar types to demonstrate proof of concept, then a larger set of parameters drawn from real-life weather radars, naval radars and airborne radars. For the set of realistic LFM parameters, two scenarios are considered. In the first scenario, the type of radar is assumed to be known and a machine learning algorithm is used to identify the chirp rate (the "mode") of the radar. Effectively, in this scenario, only subsets of the parameter set are considered. This is equivalent to identifying the sub-types of radars in an environment where it is known that only one kind of radar would be present but the variety within the radar is unknown. In the second scenario, both the type and the mode of the radar are assumed to be unknown and a machine learning algorithm is used to identify both the radar type and the mode simultaneously. The effect of not having the whole pulse for classification will also be investigated. Testing of the classifier is done with an incomplete pulse. Further, to test the robustness of the classification system, testing is carried out with radar LFM pulses whose chirp parameters are slightly deviant from the known set.

Four well-known traditional machine learning algorithms are considered in this work since classification is done with limited training data and computing power. Emerging learning architectures such as deep learning architectures and their variants are not considered. They will be considered in future research. The research in this thesis work is based entirely on simulations. (Due to the COVID-19 pandemic, collection of real data was not possible.)

\subsection{Contributions of the thesis}

The following are the contributions of the thesis:

- This is the first technical work that addresses the problem of classifying chirp rates and radar types using a single pulse waveform under poor SNR conditions using a bank of matched filter outputs.

- Unlike other papers in the literature, realistic parameters of the radars are used in this research, i.e., real world radar type waveforms were simulated in this study for both training and testing. 
- Robustness study of the system were also carried out using variants of real world radar waveforms.

The following peer reviewed publications have resulted from this research work.

- Conference Paper

- A. Young, D. Luong, B. Balaji, and S. Rajan, "Machine Learning Approach to Chirp rate Estimation of LFM Radars," Integrated Communications, Navigation, and Surveillance (ICNS), September 2020.

- Report

- A.Young, C. Livingstone, "Monitoring and Characterisation Receiver Signal Simulator - Volume 1 Conceptual Design," Defence Research and Development Canada, Ottawa Research Centre, March 2019.

- Invited Oral Presentation

- A. Young, "Wireless Communications and Sensing for Space-based Applications - The Problem Space," IEEE Global Signal and Information Processing (Global SIP) Workshop on Wireless Communications and Sensing for Space-based Applications, November 2019.

\subsection{Thesis Outline}

The following is the organization of the thesis:

- Chapter 1 introduces the problem of detecting LFM radar signal parameters for low Signal to Noise Ratio (SNR) conditions and how machine learning algorithms are viable techniques to perform that parametric characterisation. The problem is outlined and thesis objectives and framework are then discussed.

- Chapter 2 provides a literature review relevant to this thesis.

- Chapter 3 provides fundamental signal processing and machine learning algorithms needed for this thesis.

- Chapter 4 details the three types of radars and their modes considered in this research work. 
- Chapter 5 provides details about the simulations and the results. Also it discusses the results and compares them against current experimental findings.

- Chapter 6 highlights the key findings of this thesis, the contributions made to this area of research and identifies future areas of investigation. 


\section{Chapter 2}

\section{Literature review}

In this chapter, we review previous work on LFM parameter estimation. This is an important problem because synthetic aperture radars, commonly found on airborne and spaceborne platforms, typically use LFM radar signals. The detection and parametric estimation of the LFM signal, specifically chirp rates, has been a long-standing problem in the radar domain.

Many current approaches are based on the sampling theorem and fractional broadening [5]. In prior work, this problem has been investigated using various signal processing methods for LFM parametric estimation, such as the Maximum likelihood estimation (MLE), Dechirp parameter estimation, the Wigner-Ville distribution (WVD) time-frequency analysis and the Fractional Fourier transform (FrFT).

Song et al. [6] estimated LFM parameters using a discrete chirp Fourier transform for SNR values of -9 to $6 \mathrm{~dB}$. The Cramér-Rao Bound (CRB) and root mean squared error (RMSE) are closely correlated at SNR values higher than $0 \mathrm{~dB}$. Zheng et al. [7] employed a technique based on parameterised centroid frequency chirp rate distribution (PCFCRD) for noisy multi-component LFM signals. Djurovic et al. [8] used time-frequency ( $\mathrm{TF}$ ) peak position to estimate the instantaneous frequency (IF) for non-parametric chirp rate estimation without assuming polynomial phase and applied this approach to high SNR values of 18, 21 and $24 \mathrm{~dB}$.

Zeng et al. [9] proposed a feature-based recognition algorithm for three kinds of IMOP signals to detect the phase discontinuities of a BPSK signal and the chirp rate of an LFM signal. Their approach estimated the chirp rate accurately at $90 \%$ for SNR greater than $5 \mathrm{~dB}$ and identified errors greater than $10 \%$ in phase for SNR values i7 $\mathrm{dB}$. The lack of a priori information was identified as a challenge in radar electronic warfare (EW) recognition. 
Wu et al. [10] applied the STFT to analyse six radar features and used them to train support vector machine (SVM) $k$-nearest neighbour classifiers to extract feature vectors from radar signals, classify activities, and detect falls. A Markov model classifier was then applied for human act recognition. The Doppler shift was used to characterise motions with each having a different frequency. It was able to classify types of falls into categories but had misclassification errors.

The Wigner-Ville Distribution (WVD) provides good estimates if the signal-tonoise ratio (SNR) is high and has high resolution in the time-frequency plane [11]. Its main disadvantages include the inability to handle multiple incoming LFM signals arriving at a radar (congested spectrum), its ineffectiveness when receiving low-SNR LFM signals, and the difficulty of interpreting the received signal when there are multiple components to that signal. The WVD uses a time-frequency decomposition of radar signals to extract key parametric information. As the noise level increases, more signal interference terms appear and the spectrum becomes blurred [12]. The WVD requires enough time and spectrum over a pulse stream to accurately characterise signals. As SNR levels decrease below $0 \mathrm{~dB}$, this capability becomes more strained.

Sirianunpiboon [13] detected weak LFM signals using parametric estimation with the TF techniques of WVD and WH in gaussian noise. The techniques had issues with low SNR values due to the dominance of cross-terms at these values. An antenna array of receivers was used to conduct the parametric estimation to find chirp rate and noted the deficiency of computational complexity at that time. WVH was also considered as a technique.

Ahmad et al. [14] used the WVD to sort LPI airborne FM radar signals by frequency and phase. The probability of correctly sorting non-LPI radars (PCS) was 100 percent at $-12 \mathrm{~dB}$ but needed higher SNR values to correctly sort LPI LFM radar signals. Zhang et al. [15] investigated the parametric estimation of LFM signals based on a theoretical approach. They noted that the WVD cross terms decreased the ability to estimate chirp rates and that WVD has trouble when multiple LFM signals exist.

Gulum et al. [16] extracted FMCW radar signal parameters using the WHTRADON transform. This TF approach shows that it can extract radar modulation parameters down to $-9 \mathrm{~dB}$ but not on a pulse-to-pulse basis. Also, in [17], a pseudoWVD was used to extract signal parameters of FMCW radar signals where the Hough 
transform (HT) identified the signal parameters to $-6 \mathrm{~dB}$. These simulated parameters were to be compared to real radar parameters at a later date.

Guner et al. [18] used the WH transform to detect LPI LFMCW signals at SNR levels from -3 to $-14 \mathrm{~dB}$. Eight methods were evaluated for the probability to detect the chirp rate and estimate if the signal were present. They achieved a $P_{C R E}$ of 0.96 for their approach at $-8 \mathrm{~dB}$. Geroleo et al. [19] detected and estimated multi-pulse LFMCW radar signals and their chirp rates using the WVH transform to search for the sawtooth LFM pattern in the TF domain. The author noted that current TF techniques that are premised on detection of a single pulse are sub-optimal and its method is computationally demanding.

Niczyporuk [20] applied the RADON transform to WVD results to detect chirps in the SNR range of 0 to $-6 \mathrm{~dB}$ without a priori information. Results showed that the receiver did not have information on signal duration or time delay and was unable to correctly detect the signal at SNR values below $0 \mathrm{~dB}$.

Bouochikhi et al. [21] applied the Teager-Huang-Hough transform to analyse multi-component LFM signals as it has the advantage that cross-spectral signal components are free and has good TF and FD resolution. They noted that the maximum likelihood estimation (MILE) method is computationally complex and that TF methods are effective for detection and estimation (WVH, WVD, MF), but the TF spectrogram method suffers from fixed time and frequency resolutions due to fixed window length and has cross-terms which limits its application to LFM parametric analysis. WVD is optimal for maximum energy concentration around IF frequency but has cross terms. The paper tests LFM signals in noise-free and noisy environments in a SNR range of 5 to $50 \mathrm{~dB}$. The FrFT can be used to estimate LFM signal parameters but the computational efficiency is dependent upon the search range and evaluation of the optimum FrFT order.

Sirianunpiboon et al. [22] used a group invariance approach to detect weak LFM signals and noted that the FrFT for low SNR values had a window length constrained by chirp rate and was SNR-limited. It was noted that a windowed chirp rate approach only detected small chirp rates at low SNR and that discrete ambiguity needs high SNR. Cheng et al. [23] used the MCR-FrFT to detect multilinear chirp signals for underwater acoustic sensor networks.

Serbes [24] used the FrFT and a Fibonacci search algorithm to estimate LFM chirp rate for a small range of chirp rates. The MSE was very low for SNR above 7 
dB. In Serbes [25], the authors identified the accuracy of an algorithm that achieves CRB down to $-7 \mathrm{~dB}$ for chirp rates 0.1 to 0.4 . They did not use ML algorithms, but their method achieved higher performance than competing algorithms with less computational cost. They noted that a key problem was estimating chirp rates in noisy environments.

Aldimashki and Serbes [26] estimated the chirp rates and frequency of airborne radars using the FrFT for the multi-component case and noisy signals at $5 \mathrm{~dB}$ for the single component case. Mean square error versus SNR was plotted for SNR values from -10 to $10 \mathrm{~dB}$. They concluded that their method incurred minimal computational cost.

Qiu et al. [27] proposed a BPSK/LFM hybrid model for signal parameter estimation of non-cooperative radar receivers in a congested environment. Their FrFT and linear TF model estimated chirp rates at SNR greater than 0 dB. Ma et al. [28] used amplitude characteristics of LFM in FrFT domain to estimate chirp parameters. They were able to detect the chirp rate at SNR values of -6 to $4 \mathrm{~dB}$ but LFM parameter estimation was best at SNRs greater than $2 \mathrm{~dB}$.

Chen et al. [29] proposed a STFT transformation algorithm to do parametric estimation of LFM signals under low SNR and simulated it in MATLAB. They indicated that their simulation approach could theoretically be applied to various SNR values but this would take a significant amount of time and computational power. They considered chirp rates of 5 to $40 \mathrm{~dB}$. Machine learning estimation was not considered due to computational and storage requirements; although it could give better accuracy. Additionally, this approach cannot conduct a true pulse-to-pulse parametric estimation. Fourer et al. [30] proposed a chirp rate and IF estimation technique with STFT for FM signals above $0 \mathrm{~dB}$.

The detection and parametric estimation of LFM signals in particular has been an issue in the radar domain, but approaches based on matched filtering have not been well explored. A matched filter is the optimum linear filter for detecting a known signal in the presence of Gaussian noise. Its performance is non-optimum for nonGaussian noise and it requires a priori information on radar emitters [31]. Salem noted that the matched filter is one of the most effective techniques to maximise the SNR of chirp radar signals but has the drawback where its sinc response sidelobe levels degrade performance [32]. 
Other work has focused on applying machine learning approaches to LFM parametric characterisation for TF analysis. Several techniques have been proposed to conduct parametric estimation of LFM signals using machine learning algorithms. Convolutional neural networks (CNNs) have been used to classify waveform types [33] and signal parameters [34]. These machine learning algorithms have been used with various types of preprocessing. For example, [10] used a short-time Fourier transform (STFT) to recognize micro-Doppler features in radar signals, then applied Markov and support vector machine (SVM) classifiers. The Wigner-Ville transform has also been used [35].

Ruiz et al. [36] used non-parametric Bayesian inference to classify images as states. It did not require a priori information and found similar results compared to a supervised SVM mode. The kappa metric was used to assess the performance accuracy of the classifier compared to SVM to properly classify 10 images. Confusion matrices were not used and the author only discussed classifier running time as the factor to properly classify images.

Lunden et al. [37] used feature vector recognition to recognise signal waveform features, such as leading and trailing edge of pulse, bandwidth, and calculated them using a TF Choi-Williams distribution for LFM signals in pulse compression radars. Bayesian classifiers were also considered. They were able to classify received waveforms as one of 8 types using their hierarchical approach.

Fischell and Schmidt [38] used a SVM classifier to estimate target aspect angle from bistatic acoustic scattering. The classifier was trained on simulated targets and appeared to be effective in classifying actual data. Seok [39] used Hidden-Markov, NN and SVM classifiers to classify active sonar targets and the full sonar LFM signal. Feature extraction was also implemented. The article notes that combining the results of multiple classifier output reduces the generalisation error.

El-Khamy and Elsayed [40] classified multi-user chirp LFM signals using wavelets. Neural networks, support vector machines and maximum likelihood estimators were used to classify the signals. The probability of a correct decision was only considered for SNR values ranging from 0 to $50 \mathrm{~dB}$. The Neural Network (NN) classifier was identified as the best classifier and indicated that signals could be classified in Gaussian white noise.

Boashash and Ouelha [41] used the WVD to assess the spread of signals in the TF domain and implemented machine learning classifiers to improve non-stationary 
signal classification thorough feature extraction. Three classifiers were used for feature extraction: SVM, Random Forest (RF), and Artificial NN (ANN). The metrics of sensitivity, specificity and total accuracy were used as metrics and the authors found that SVM generalizes well and has good accuracy. They propose that for a multi-class problem, accuracy should be used to evaluate feature selection.

$\mathrm{Su}$ et al. [42] did parameter estimation for chirp signals using a complex deep CNN that uses CV DNN processor for feature extraction. The chirp rate was varied from 0.04 to 4 in steps of 0.04 with no overlapping values in training and testing data to avoid overfitting. SNR values were superimposed on the data ranging from $-20 \mathrm{~dB}$ to $100 \mathrm{~dB}$ in steps of $10 \mathrm{~dB}$. Simulation results indicated that the approach was more accurate than WVH transform and that there were benefits to using deep learning systems for signal parameter estimation.

Lee et al. [43] investigated jamming prediction for radar signals using a combination of ML classifiers (SVM, NN, and RF) to perform feature extraction and classify the signals. They obtained an accuracy of $98.46 \%$ for NN with extracted features, but $99.36 \%$ for LSTM without feature extraction. However, LSTM took longer to train than NN.

Gao et al. [44] performed fusion image radar signal feature extraction and modulation recognition using NN to extract features and SVM to classify the image. WVD was not viable due to cross terms. The confusion matrix (CM) showed an accuracy of $94.3 \%$ at $2 \mathrm{~dB}$ for RSR feature extraction for the image. The authors noted that $90 \%$ recognition at greater than $0 \mathrm{~dB}$ is very good performance.

Wang et al. [45] proposed a hybrid ML approach to extract features of seven modulation types, including LFM signals. STFT was used in the first layer to conduct feature extraction while NB and SVM classifiers were used to classify the modulation waveforms. The authors only looked at SNRs from $10 \mathrm{~dB}$ to $30 \mathrm{~dB}$ and achieved accuracy rates of $94.42 \%$ at $10 \mathrm{~dB}$ for LFM using this approach. With noise, it was $93.42 \%$ for SVM and less than $80 \%$ accuracy at $0 \mathrm{~dB}$.

Noone [46] used SVM to classify radar signals into various categories. Two signals were recorded at distances of $3 \mathrm{nmi}$ and $6 \mathrm{nmi}$ for three pulse distribution (PD) modes, and the mean pulse characteristic for each category was calculated at each distance. A SVM was used to classify and train on a series of pulses (4340 pulses for three PD modes). Their algorithm was susceptible to SNR changes, could not handle noisy environments, and needed other pulse characteristics to categorise the complete 
pulse stream.

Kwon et al. [47] used deep neural networks to recognise micro-Doppler radar signals of movements using FFT results as feature vectors. MLP and DNN were used for multi-class classifiers. The MLP had $89.8 \%$ accuracy for the test set while the DNN had $97.2 \%$ accuracy. The results were presented using confusion matrices. The authors stated that the DNN can be applied as an effective approach without complex feature extraction.

Hong et al. [48] classified radar signals using CNN. They wrote that a librarybased method cannot properly classify radar signals due to the complexity of new radar waveforms, but a ML could. The CNN classifier received radar pulse radar parameters of RF, PRI, pulse amplitude (PA), PW and estimated a PW for each signal pulse of the total signal. The proposed method assumed an ideal noise environment for the pulse stream. The probability of success is $90 \%$ after 30 iterations and $93 \%$ after 200 .

Carretero et al. [49] trained an ANN on CFAR window attributes and found that this approach outperforms traditional CFAR methods. They performed Monte-Carlo simulations at SNR values from -10 to $30 \mathrm{~dB}$.

Ren et al. [50] considered LFM, FSK, BPSK, CW and QPSK waveforms as modern radars have more sophisticated waveforms and inter-pulse information may not be enough to separate received pulses according to their source emitters. They used signal feature extraction using wavelets to simplify classifier and computational complexity by generating smaller-dimensional feature vectors. They simulated 150 samples at SNRs of 5, 10, 15, and $20 \mathrm{~dB}$ for a total of 3000 signals. They defined 34 features such as instantaneous frequency and bandwidth. However, they considered only one chirp rate. Their best accuracy rate was $95.26 \%$ at $5 \mathrm{~dB}$.

Kurdzo et al. [51] used a NN with four hidden layers to optimise a set of NLFM waveforms and increase their resolution via interpolation. Bandwidth and pulse length were used as inputs to the NN classifier. The need to determine waveform parameters on a pulse to pulse basis was noted for future work.

Mughai and Kim [52] conducted signal classification and jamming detection of wideband radios using a Naïve Bayes (NB) classifier to classify the signal at different jamming levels. Compressive sensing recovered the wide-band spectrum; they then trained a NB classifier in feature extraction of spectral features.

$\mathrm{Xu}$ et al. [53] applied sparse Bayesian techniques for radar target recognition to 
binary and multi-tasks classification problems. They used a LV-SVM to perform binary classification of each task. Three airborne radars were used with the same frequency and bandwidth for 524 test samples and 500 training samples, a total of 4721 samples. It was noted that the more samples there are, the better the classifier recognises the radar and that the performance was better than that of kernel SVM.

Selim et al. [33] used a CNN ML algorithm to detect radar signals in a spectrum sharing situation, in the presence of signal interference (i.e., congested spectrum) to infer spectrum occupancy and achieved $99.6 \%$ accuracy in detecting RF signals. They looked at LFM Barker BPSK phase modulation (PM) and pulse carrier (PC) for their intra-pulse modulation (IPM). They characterised radar pulses and assumed that all pulses were the same width, transmitting pulses every $1 \mathrm{~ms}$. A trained CNN classifier was tested at low SNR values of 2 to $20 \mathrm{~dB}$ and achieved $99.6 \%$ accuracy.

Kong et al. [34] classified radar signals with a CNN ML classifier to model the real part of the signal waveforms in the time domain. They generated eight types of waveforms for eight radar types, converted radar signals into the time domain using STFT, and then extracted the time-frequency distribution in frequency domain. A CNN extracted radar signal characteristics. The authors show that CNN learns more about features after STFT. Their approach approximates the radar signal with a data model for the algorithm to recognise, then performs feature extraction. There were difficulties in correctly identifying unknown radar signals. According to the authors, they selected CNNs as they have strong nonlinear fitting and self-learning abilities. Learning rules are simple and easier to implement. However, CNNs have more difficulty recognising radar signals.

Wang et al. [54] used support vector clustering with time-frequency analysis and the RADON transform of multi-component LFM signals to detect signal components using the WVD, ambiguity function and wavelets. Two experiments were conducted to detect chirps at SNRs above $-7 \mathrm{~dB}$. The assertions that chirp rates could be classified above $-8 \mathrm{~dB}$ were not substantiated with performance metric values or SNR results in the paper.

Finally, Caroni and Souryal [55] evaluated the performance of matched filters to detect radars in the $3.5 \mathrm{GHz}$ band and selected a ML approach to filter out inband interference. A priori knowledge of PRI and pulse duration was assumed. They implemented a bank of MFs over the entire baseband. Results showed that the magnitude detector performed well at an SNR of $5 \mathrm{~dB}$ in Gaussian noise as its 
performance exceeded commercial devices.

\section{$2.1 \quad$ Summary}

The extensive literature review indicates that much work has been performed on LFM parameter estimation and classification using conventional techniques such as the Wigner-Ville transform and other time-frequency methods. To a lesser extent, matched filtering has been studied as well. On the other hand, a good amount of work has been devoted to applying machine learning algorithms to radar signal classification as well. However, very little work has been done on combining the two approaches. Thus, the approach used in this thesis, namely the use of a bank of matched filters combined with machine learning algorithms to classify a low-SNR radar signal is novel. This approach is so novel, in fact, that only a preliminary investigation is possible in this thesis. 


\section{Chapter 3}

\section{Radar Signal Processing and Machine Learning}

\subsection{Introduction}

This chapter provides a quick overview of relevant aspects of radar signal processing and machine learning algorithms needed for understanding this thesis.

The standard radar waveform that is used in many practical systems, linear frequency modulation (LFM), will be defined, as well as the theory of our matched filter approach used in our model and the Wigner-Ville distribution (WVD) that we will use a basis of comparison.

Finally, four basic machine learning algorithms will be explained, along with key performance metrics that will be used to evaluate the model.

\subsection{Fundamentals of Radars and Radar Intercept Receivers}

A radar is an electromagnetic system that detects and locates objects by transmitting a waveform. A radar consists of a radio frequency (RF) front end, i.e., the receiving and transmitting antennae, and the system processing components to infer the presence/absence of a target. The radars are typically installed on static (i.e., weather radars) or mobile platforms (i.e., airborne, automotive, marine, or space radars). The target needs to be in the antenna main beam in order to be detected (highest SNR value). Radars use techniques, such as pulse compression, to achieve higher range 
resolution without requiring shorter pulses and maintain the energy of longer pulses. Continuous waveforms are also used to take advantage of the Doppler frequency, $f_{d}$, shift to separate received signal from echoes due to environmental clutter.

An intercept receiver is a radar receiver which is typically wideband and is used to detect emissions of transmitting radars.

The radar intercept receiver equation is used to infer signal-to-noise ratio of received signals from transmitting radars [56]. The power density, $P_{d}$ of a radar transmitter at a distance, $R$, from the radar intercept receiver is given by equation1:

$$
P_{d}=\frac{P_{r}}{4 \pi R^{2}}
$$

Directional antennas are employed by radars to direct the radiated energy in a given direction. Their antenna gain, $G_{t}$, is a measure of the radiated power in a direction compared to that of an isotropic antenna. The power density received by the intercept receiver with an antenna with gain, $G_{r}$, is thus given by

$$
P_{d}=\frac{P_{t} G_{r}}{4 \pi R^{2}}
$$

The receiving antenna with effective area, $A_{e}$ will capture part of this echoed power and the received power will be given by equation 3 :

$$
P_{d}=\frac{P_{t} G}{4 \pi R^{2}} \frac{\sigma A e}{4 \pi R^{2}}
$$

A radar's ability to detect a target's signal is constrained by the energy of the ambient noise that occupies the same electromagnetic spectrum as the radar and that interferes with signal detection. Noise sources vary from inherent receiver sources to other radars. This noise power is a composite value of the thermal noise and other noise factors. Suffice to say, that for the purposes of this thesis, we assume that the noise power is given by

$$
N_{0}=k T_{0} B F_{n}
$$

where $N_{0}$ is the noise from the receiver, $T_{0}$ is the standard environmental temperature of 290 degrees kelvin, and $B$ is the radar intercept receiver intermediate frequency (IF) bandwidth, and $\mathrm{k}$ is Boltzmann's constant. 
Therefore, the signal-to-noise ratio (SNR) is given by

$$
\mathrm{SNR}=\frac{P_{t} G}{\left(4 \pi R^{2}\right)^{2}} A_{e} \frac{1}{k T_{o} B F_{n}} .
$$

The signal that arrives at a radar receiver from a transmitting radar is comprised of the radar signal and various sources of noise, such as clutter, other interference sources and jamming. In this thesis, the effects due to clutter and other interferences are not considered.

\subsection{Linear frequency modulation (LFM) modula- tion}

Conventional pulsed radars transmit narrowband bandpass signals. The equation for an individual transmitted radar pulse is written as:

$$
x_{\mathrm{a}}(t)=a(t) \sin \left(2 \pi f_{c} t+\theta(t)\right)
$$

where $a(t)$ is the constant amplitude pulse envelope, $f_{c}$ is the radar carrier frequency, and $\theta(t)$ represents either a constant phase or phase modulation of the pulse, The received radar signal amplitude is directly related to the received power in the radar intercept range equation, $P_{r}$.

Most radars use either linear frequency modulation (LFM) or Non-linear Frequency Modulation (NLFM). This thesis will focus on the case of radars that transmit LFM waveforms. In general, LFM signals can be written in the following form:

$$
x_{\mathrm{LFM}}(t)=\exp \left[j \phi_{0}+2 \pi j\left(\alpha t+\frac{\beta}{2} t^{2}\right)\right],
$$

where $\phi_{0}$ is the initial phase, $\alpha$ is the starting frequency, and $\beta$ is the chirp rate, i.e.,

$$
\beta \in\left\{\beta_{1}, \ldots, \beta_{m}\right\}
$$

where $m$ is the number of possible chirp rates of the LFM signal. In the context of transmitting radars, these chirp rates correspond to the radar operating modes. The goal of this thesis is to determine the chirp rate for an arbitrary LFM signal where the chirp rate is unknown, but is drawn from a set of known chirp rates. Without 
loss of generality, we can set $\phi_{0}=0$ for the purpose of this thesis. The parameter $\alpha$ is the carrier frequency of the transmitting radar.

Assuming that we have some a priori knowledge the emitter, say due to regulatory requirements, we can set $\alpha=0$. In practice, at the intercept receiver side, this would be equivalent to demodulating the signal to baseband.

At the intercept receiver side, we assume that all the signals involved have been sampled respecting the Nyquist rate to yield discrete-time signals. Thus, the ideal LFM signal $x_{\mathrm{LFM}}(t)$ would be sampled at a constant sampling frequency $f_{s}$, yielding the discrete-time signal

$$
x_{\beta}[n]=\exp \left[2 \pi j \cdot \frac{\beta}{2}\left(\frac{n}{f_{s}}\right)^{2}\right], \quad n=0, \ldots, N-1
$$

where $N$ is the length of the sampled signal. In general, a received LFM signal may be corrupted by noise, yielding

$$
x[n]=x_{\beta}[n]+w[n] .
$$

For the purposes of this thesis, we assume that the noise, $w[n]$, is a complex-valued Gaussian white noise .It is further assumed that any incoming signal contains at most one LFM signal.

The problem we will consider may be stated as follows: given a signal of the form $x_{\beta}[n]+w[n]$, determine $\beta$ drawn from a predetermined set of $m$ chirp rates $\left\{\beta_{1}, \beta_{2}, \ldots, \beta_{m}\right\}$.

\subsection{Wigner-Ville Distribution}

The Wigner-Ville Distribution (WVD) is one of the key techniques in radar signal processing as it represents a signal in both the time and frequency domains. It can be used to determine the presence of a specific LFM signal with its modulation rate in high SNR scenarios [3]. The WVD uses the autocorrelation function to calculate the power spectrum of a signal and preserves the highest energy of a linearly modulated signal in the time-frequency domain. 
The WVD of a continuous input signal, $\mathrm{x}(\mathrm{t})$, is:

$$
W_{x}(t, \omega)=\int_{-\infty}^{\infty} x\left(t+\frac{\tau}{2}\right) x^{*}\left(t-\frac{\tau}{2}\right) e^{-j \omega t} d \tau
$$

where $t$ is the time variable, $\omega=2 \pi f$ is the angular frequency, and * represents the complex conjugate of the input signal, $x(t)$.

$$
W_{x}(t, f)=\int_{-\infty}^{\infty} x\left(t+\frac{\tau}{2}\right) x^{*}\left(t-\frac{\tau}{2}\right) e^{-j 2 \pi f t} d \tau
$$

The WVD transformed to the Fourier domain is:

$$
W_{x}(\omega, t)=\frac{1}{2 \pi} \int_{-\infty}^{\infty} X\left(\omega+\frac{\omega_{0}}{2}\right) X^{*}\left(\omega-\frac{\omega_{0}}{2}\right) e^{-j \omega_{0} t} d \omega_{0}
$$

It can be noted from these two equations that:

$$
W_{x}(t, \omega)=W_{x}(\omega, t)
$$

For a discrete signal, $x(t)$ sampled at sampling frequency $f s$ with $N$ samples, the equation is:

$$
W_{x}(n, k)=\sum_{m=-N}^{N} x\left(n+\frac{m}{2}\right) x^{*}\left(n-\frac{m}{2}\right) e^{-j 2 \pi k m / N}
$$

In this thesis, we will compare the performance of the proposed machine learning technique with that of the WVD and show that WVD does not work well in low SNR conditions.

\subsection{Machine Learning algorithms and perfor- mance metrics}

Machine Learning attempts to obtain models from the data by learning the underlying relationships. Machine learning algorithms may also be viewed as the use of statistical methods, and algorithms to make classifications or predictions, uncovering key relationships that group certain data together. These relationships subsequently lead to decision making.

There is a wide variety of machine learning algorithms ranging in flexibility and 
complexity that can be used to classify radar chirp rates. Each algorithm has its own assumptions and, based on the underlying assumptions and methodology used to capture the hidden information, the complexity of the resulting classifier will differ. In this thesis, four well known machine learning algorithms are considered to classify the chirp rates. They are Decision Tree (DT), Naive Bayes (NB), Support Vector Machine (SVM) and Random Forest (RnF). These four machine learning algorithms or classifiers are described in the following subsections.

\subsubsection{Support Vector Machine (SVM) Classifier}

Support vector machines (SVM) are binary classifiers. In a binary classifier, one can choose several hyperplanes to separate the classes. A SVM classifier would produce the best hyperplane that maximizes distance between the data points in the two classes. This line is referred to as a maximum-margin hyperplane and is used to separate data points into two classes [1]. The further away from the hyperplane that a data point is, the larger that margin between data point and the hyperplane, the higher the probability that a correct prediction has been made in classification. The points closest to the separating hyperplane are known as support vectors. The classifier's goal is to maximise the distance from the hyperplane to the support vectors.

This maximization leads to a good classification with good confidence even for unseen data points. In other words, SVM's strength is in its ability to classify data points that are outside the classifier training set [57]. This is termed as generalization in machine learning. A multi-class classification problem can be reduced to a set of binary classification problems (using a one-vs.-one or a one-vs.-all strategy) as long as there are no dependencies between any two clases.

An SVM's learning phase consists of finding a set of parameters by solving a Convex Constrained Quadratic Programming (CCQP) problem. A set of hyperparameters is tuned to find the SVM that provides the most optimal performance in classifying the set of data. This is known as the model selection phase where the chosen model is characterised by the lowest estimated generalisation error [58].

The equation for the hyper-plane is

$$
g(x)=w^{T} x+w_{0}
$$

where $w_{0}$ is the weight vector and $w_{0}$ offset. The distance from a data point to the 


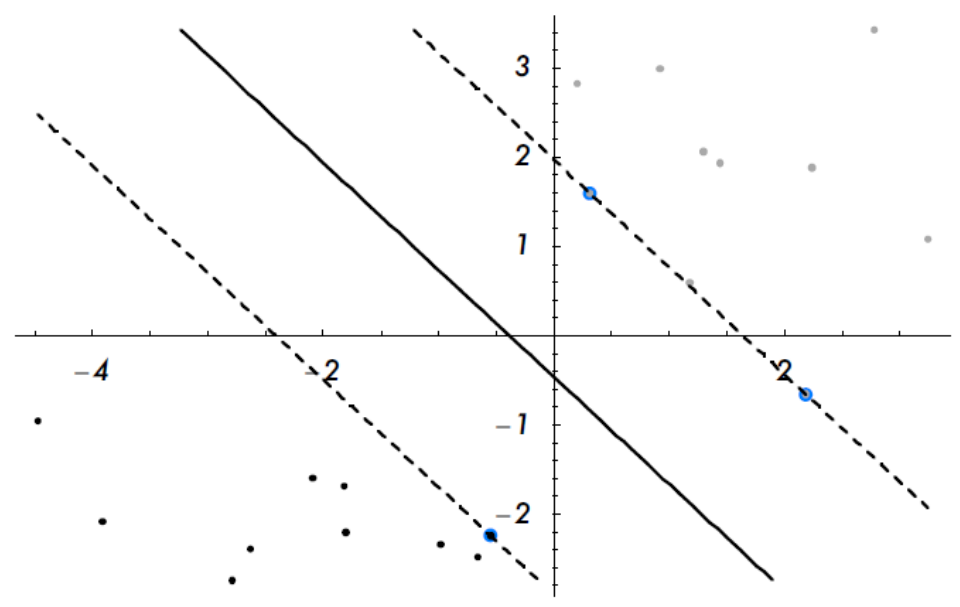

Figure 1: Support Vector Machine Hyperplane [1].

hyperplane is defined in Equation (17) and is known as the margin

$$
b=\frac{\left|w^{T} x+w_{0}\right|}{\|w\|}
$$

In order to determine where the optimal placement of the hyperplane is for the data set comprised of linearly separable values, the SVM classifier allocates data points into separate feature domains split by the hyperplane where:

$$
u=w^{T} x+w_{0}<0 \text { if } x \in C=-1,
$$

and

$$
u=w^{T} x+w_{0}>0 \text { if } x \in C=1 .
$$

In the above equation $C$ represents the class.

If a point is either quite far from the hyper plane on the positive or negative side, then $u$ will be a large positive number. Thus, for the binary problem, we can assign points to either Class 1 or Class -1 . The points with the smallest margins form the hyper-plane and will be referred to as the support vectors. Once the points with the smallest margin are found, SVM then maximises the margin so that $u=1$ and we have the maximum of $\frac{1}{\|w\|}$. For an $N$ class problem, there will be $N$ hyper-planes and regions defined. The complexity of the SVM classifier is proportional to the number of SVM and not the feature space itself. 
The procedure implemented in Wolfram Mathematica is as follows:

- Transform the data to the format of the SVM package.

- Scale the data to a linear range of $[-1,+1]$ or $[0,1]$.

- Apply the relevant kernel to the data.

- Determine the best values for $C$ and $\gamma$ through cross-validation.

- Test the data.

- Run the trained classifier on the data sets.

\subsubsection{Naive Bayes (NB) Classifier}

Bayes theorem determines the conditional probability of an event $A$ when another event $B$ has occurred, i.e.,

$$
P(A \mid B)=P(B \mid A) \times \frac{P(A)}{P(B)}
$$

where $P(B \mid A)$ represents the probability of event $B$ assuming that event $A$ has occurred, $P(A)$ is the probability of event $A$, and $P(B)$ is the probability of event $B$.

Let $X=\left\{x_{1}, \ldots, x_{m}\right\}$ be the set of of $m$ possible features and $y$ be the associated class variable. Then, using Bayes' theorem

$$
P(y \mid X)=\frac{P(y, X)}{P(X)}=\frac{P\left(x_{1} \mid y\right) P\left(x_{2} \mid y\right) \cdots P\left(x_{n} \mid y\right) P(y)}{P\left(x_{1}\right) P\left(x_{2}\right) \cdots P\left(x_{n}\right)} .
$$

Since the denominator is a known quantity, the class $y$ with maximum probability is given by

$$
\tilde{y}=\operatorname{argmax}_{y} P(y) \prod_{i=1}^{n} P\left(x_{i} \mid y\right) .
$$

The probability distributions above could be Bernoulli, Multinomial or Gaussian. In the binary classification case, the distribution is Bernoulli.

Wolfram Mathematica Naive Bayes variant implements Baye's theorem where $P($ class $\mid x) \propto P($ class $) P(x \mid$ class $)$ and assumes that each feature, $\mathrm{x}$, is independent given a class. The class probabilities are $P($ class $\mid x) \propto P($ class $) \prod_{i=1}^{n} P\left(x_{i} \mid\right.$ class $)$. 
$P\left(x_{i} \mid\right.$ class $)$ is the probability distribution of the feature $x_{i}$ given a class and $\mathrm{P}($ class $)$ is the prior probability of the class. Both distributions are estimated from the training data. Distributions are modeled using a piecewise-constant function. A regularisation parameter, "SmoothingParameter", can be defined to smooth out the function [59].

\subsubsection{Decision Tree (DT) Classifier}

Decision Trees are a non-parametric supervised learning method used for classification. The decision tree algorithm seeks to learn simple decision rules from training data and then uses those rules to predict the class of the test data.

Let the training data be denoted by $T \in \mathbb{R}^{N \times D}$, where $N$ is the number of samples and $D$ is the number of features. Also, let the label matrix be denoted by $L \in \mathbb{R}^{N \times K}$, where $K$ is the number of labels/classes. A decision tree recursively partitions the space of the training data such that the samples having the same class labels will be grouped together.

Consider the data at node $m$ that is represented by $T$. For each candidate split $\theta=\left(j, t_{m}\right)$, where $t_{m}$ is the threshold at node $m$ for feature $j$. The data is then partitioned into $T_{l}$ and $T_{r}$ subsets:

$$
\begin{aligned}
T_{l}(\theta) & =(T, y) \mid T_{j} \leq t_{m}, \\
T_{r}(\theta) & =X \backslash T_{l}(\theta)
\end{aligned}
$$

A function called impurity function $F(T)$ is introduced. Two common choices for the impurity functions are

$$
\begin{aligned}
& F\left(T_{m}\right)=1-\sum_{i=1}^{K} p_{i}^{2} \\
& F\left(T_{m}\right)=-\sum_{i=1}^{K} p_{i} \log _{2}\left(p_{i}\right),
\end{aligned}
$$

where

$$
p_{i}=\frac{1}{N_{m}} \sum_{t_{i} \in R_{m}} I\left(y_{i}=k\right)
$$

where $I$ is the indicator function. Equation (24) is called the gini index while equation 
(25) refers to entropy. The parameters $\theta=\left(j, t_{m}\right)$ are selected such that at node $m$ the impurity at the left and right nodes are minimized. This is accomplished via

$$
Q\left(T, j, t_{m}\right)=F(T)-\frac{\left|T_{l}\right|}{|T|} F\left(T_{l}\right)-\frac{\left|T_{r}\right|}{|T|} F\left(T_{r}\right)
$$

This is carried out recursively such that the maximum allowable depth of the tree is reached and the number of samples in a node is either 1 or less than the minimum number of samples split.

\subsubsection{Random Forest (RnF) Classifier}

Random forest $(\mathrm{RnF})$ is an ensemble learning method for classification and regression that operates by constructing a multitude of decision trees, otherwise known as an ensemble [60]. The forest prediction is obtained by taking the most common class or the mean-value tree predictions [61].

In order to have a better classification result (reduced variance) different techniques such as bagging and boosting are employed. To reduce the variance, a random forest bags number of decision trees in order to arrive at a classification result. Subsamples are drawn with replacement so that the sub-sample size is the same as the original input sample size. In addition, sub-samples have a random subset of features. Although, the bias of the forest may increase, averaging over the ensemble will reduce the variance leading to a better model.

\subsection{Machine Learning Metrics}

This section provides a brief summary of the metrics that are commonly used to evaluate machine learning algorithms.

A confusion matrix [62] is a $M \times M$ matrix used for evaluating the performance of a classification model where $M$ is the number of target classes.

The entries in the confusion matrix for a binary classifier are defined in terms of the following quantities:

- The positive $(\mathrm{P})$ and negative $(\mathrm{N})$ labels represent the predicted outcomes,

- The true $(\mathrm{T})$ and false $(\mathrm{F})$ labels represent the actual outcomes, 
- TP represents the number of times that an actual outcome is true but the predicted outcome is true,

- FP represents the number of times that an actual outcome is false but the predicted outcome is true,

- FN represents the number of times that an actual outcome is false but the predicted outcome is false, and

- TN represents the number of times that an actual outcome is true but the predicted outcome is false.

The confusion matrix for a binary classifier is defined as:

$$
\left[\begin{array}{cc}
\mathrm{TP} & \mathrm{FN} \\
\mathrm{FP} & \mathrm{TN}
\end{array}\right]
$$

It shows the actual values of True Positive (TP), True Negative (TN), False Positive $(\mathrm{FP})$ and False Negative (FN) rates obtained using the machine learning classifier. FP errors are known as Type I errors while FN errors are defined as Type II errors in statistical detection theory. For a $M$-ary classification problem, the confusion matrix has $M \times M$ elements, where each element can be designated as $n_{i j}$ where $i$ identifies the row and $j$ identifies the column. Each element indicates the number of times that data from class $i$ that has been classified as belonging to class $j$.

The total number of cases, $N$, in the $M$ class matrix is:

$$
N=\sum_{i=1}^{M} \sum_{j=1}^{M} n_{i j}
$$

Confusion matrices can also present the percentage values instead of the number of cases. In this thesis, the number of cases will be represented in the confusion matrices. It should be noted that an asymmetric confusion matrix can reveal a biased classifier.

The performance of the confusion matrix is evaluated from the categorisation of the results for the True Positive (TP), True Negative (TN), False Positive (FP) and False Negative (FN) rates. From [63], we can define the following performance metrics to evaluate the performance of the $M=2$ confusion matrix. 
The misclassification rate is the proportion of negatives cases that were incorrectly classified as: positive and is defined as

$$
\text { Misclass }=\frac{F P+F N}{T N+F P+F N+T N}
$$

The specificity rate is defined as the proportion of negatives cases that were classified correctly and is calculated as:

$$
\text { Spec }=\frac{T N}{T N+F P}
$$

The accuracy (acc) is defined as follows:

$$
\mathrm{acc}=\frac{T P+T N}{T P+F P+F N+T N}
$$

The error rate is another common parameter, ER, used to assess the performance of a classifier and is $\mathrm{ER}=1-$ acc.

The accuracy determined using Equation (32) may not be an viable performance measure when the number of negative cases is much greater than the number of positive cases [64]. It should be noted that the accuracy (acc) and the error rate, ER, depend upon the number of classes and on the total number of data, $N$.

The F1-score is a harmonic mean of Precision and Recall, and so it gives a combined idea about these two metrics and is defined as follows [65]:

$$
F 1=2 * \frac{\text { prec } * \text { recall }}{\text { prec }+ \text { recall }}
$$

The F1-score is maximum when Precision is equal to Recall. Based on the data, there will be performance metrics that will better reflect the classifier performance due to the worst case scenario and the error type that needs to be avoided. These values can then be inserted into Equation (33) to give us the F1 values for each class.

The above definitions can be extended for $M$-ary classification. An $M$-ary classification problem can be considered as a series of $M$ binary classification problems so that the above definitions can be utilized.

In this thesis, the confusion matrix has been selected as the means to report results in our $M$-class classification problem as it is possible to observe the relations between the classifier outputs and the true outputs. The accuracy, recall, precision, 
CHAPTER 3. RADAR SIGNAL PROCESSING AND MACHINE LEARNING 28

and F1 values will be calculated for the confusion matrices of each classifier to assess their performance. 


\section{Chapter 4}

\section{Some Common Radars}

\subsection{Introduction}

Three radar types namely, the weather, the air-surveillance and the marine radars are considered in this work. These radars are typically found in harbours and airports. Each of the radars can operate in several modes. In this chapter, we discuss the three radar types and their realistic values for the radar parameters in the different modes of operation.

\subsection{Weather radars}

Ground-based weather radars are widely distributed and come in a variety of forms to support differing observational requirements. Deployed weather radars fall into two broad categories: (i) high power, fixed, long-range radars in the $\mathrm{S}$ band and C-band and (ii) medium to low-power short-range radars in the C-band and X-band (primarily $\mathrm{X}$ band). The high power long-range radars are tower-mounted large systems that radiate between $250 \mathrm{~kW}$ and $1.1 \mathrm{MW}$ short (0.8 to $2.0 \mu$ seconds) pulses at horizontal and/or vertical polarization at pulse rates between $300 \mathrm{~Hz}$ and $500 \mathrm{~Hz}$. For weather radar applications, cross-polarized radar returns are generally insignificant and horizontal and vertical linear polarizations are sometimes simultaneously transmitted. Some systems use circularly polarized transmissions. The medium to low-power short-range radars are often transportable systems that radiate between $100 \mathrm{~W}$ and $25 \mathrm{~kW}$ short pulses (0.2 to $1.2 \mu$ seconds) at both polarizations that radiate at pulse rates between $1 \mathrm{kHz}$ and $2.5 \mathrm{kHz}$.

Traditionally, weather radars use circular parabolic dish antennas. Long-range 
radars are mechanically scanned in azimuth between 3 and 6 RPM and are stepped in elevation over a period of several (approximately 5) minutes. The antenna elevation angle is fixed or varies slowly for each azimuth scan. A demanding elevation [66] scanning strategy for a WSR-88D radar is shown in Figure 2. The WSR-88D uses pulse repetition rates between $320 \mathrm{~Hz}$ and $1300 \mathrm{~Hz}$ and, for long-range (small elevation angles) alternates between $4.57 \mu$ seconds pulses at $320 \mathrm{~Hz}$ and $1.57 \mu$ second pulses at $1280 \mathrm{~Hz}$ on alternate azimuth scans.

Figure 2: WSR 88D Weather Radar scanning pattern

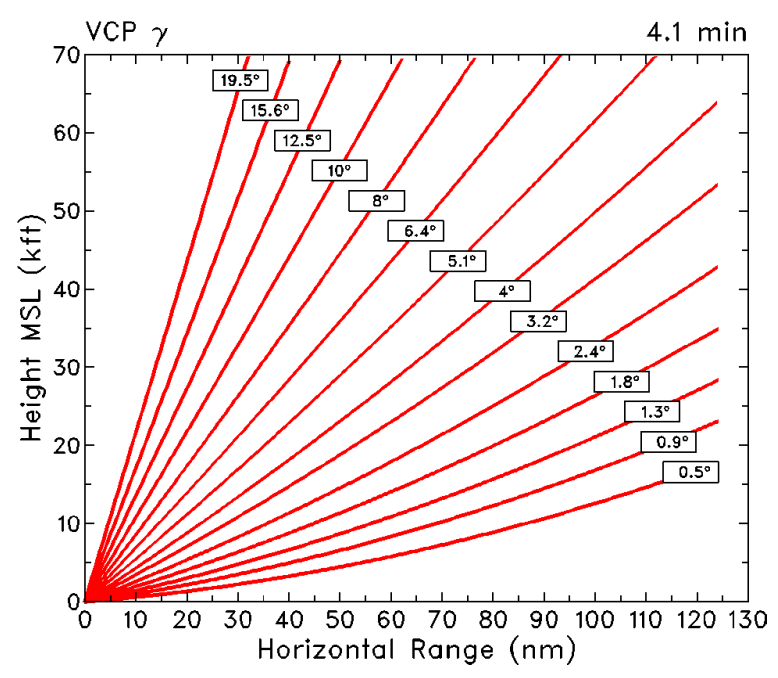

Short to medium range weather radars are typically portable systems that supply local weather data within $80 \mathrm{~km}$ (often less than $60 \mathrm{~km}$ ) range of the radar site. Traditional short-range weather radars also use parabolic dish antennas with mechanical azimuth and elevation scans. Typical azimuth scan rates vary from 3 RPM to 6 RPM and many of the operational modes and data acquisition strategies used for larger systems are also used for the smaller systems. Because of the shorter range requirement, antenna beam widths up to $3^{\circ}$ are useful. Some of the most compact systems are specialized for rain observations. Many of the short range radars in common use magnetron transmitters and radiate peak powers between $10 \mathrm{~kW}$ and $50 \mathrm{~kW}$. As with the large long-range radars, transmitted signal and beam scanning requirements are driven by meteorological observation requirements [66], [67].

More modern portable weather radars use pulse compression technology to reduce the required peak radiated power and use solid state transmitters [68]. Typical transmitted powers are 100 to $500 \mathrm{~W}$ but one at $3.5 \mathrm{~kW}$ peak power operating in 
C-band is also available [69]. Some recent systems use single polarization flat plate array antennas with mechanical steering, other systems come with single array, and with mechanical azimuth scans and electronic elevation beam steering. Few weather radars also use multiple electronically steered phased array antennas panels [70].

In this thesis, we use the NEXRAD WSR 88 S-band long range system [71] as the example for our weather radar. The system uses a rotating, circular, centerfed parabolic dish antenna, operates at frequencies in the vicinity of $3.0 \mathrm{GHz}$ and alternately transmits long and short CW pulses. Typical operating parameters [71] are given in Table 2. Additional information can be found in [66].

\subsection{Marine radars}

Marine navigation radaras operate in the $\mathrm{S}, \mathrm{C}$ and $\mathrm{X}$ bands. The International Telecommunications Union (ITU) recognizes three navigation radar classes: the International Maritime Organization (IMO) and fishing boat class, the river class and the pleasure-craft class. Their frequency ranges are detailed in Table 3.

Following the discussion in [1], the radar properties for each marine radar class are given in Tables 4, 5 , and 6 . These tables provide the typical features of each radar class.

All of the radars shown in Tables 4, 5 ,and 6 use uncoded incoherent (magnetron) pulse transmissions. More recent radars new marine radar systems [72] show a transition to coherent, internally coded pulse transmissions combined with a reduced-power range-compression technology.

Under IMO rules, all vessels greater than 200 tons and all passenger vessels require an X-band navigation radar while vessels greater than 3000 tons require an additional S-band radar (or, with permission, a second independent X-band radar). Marine navigation radar antennas are mounted on or above a vessel's bridge roof (and often on a ship's mast) to maximize usable radar range and to minimize human exposure to microwave radiation. For small pleasure craft, the antennas are typically higher than $2.5 \mathrm{~m}$ above sea level (ASL). For large vessels heights above $10 \mathrm{~m}$ ASL are common. Common marine navigation radar antennas radiate signals into a fan beam that 


\begin{tabular}{|c|c|}
\hline Radar Parameter Feature & Value \\
\hline Range resolution (m) & 235 \\
\hline Detection $Z 10(\mathrm{dBz})$ with RCS at $460 \mathrm{~km}$ & -23 to $-25 \mathrm{~dB}$ \\
\hline \multicolumn{2}{|l|}{ Angular Coverage } \\
\hline Azimuth & Full circle or sector \\
\hline Elevation & Operational limits: $-1^{\circ}$ to $20^{\circ}$ \\
\hline \multicolumn{2}{|l|}{ Antenna } \\
\hline Type & S-band, center-fed, parabolic dish \\
\hline Reflector Aperture & $8.54 \mathrm{~m}$ diameter; circular \\
\hline \multirow[t]{2}{*}{ Beam width (one-way, 3dB) } & $0.96^{\circ}$ at $2.7 \mathrm{GHz}$ \\
\hline & $0.88^{\circ}$ at $3.0 \mathrm{GHz}$ \\
\hline Gain & $45.8 \mathrm{~dB}$ at $2.85 \mathrm{GHz}$ (midland) \\
\hline Polarisation & Linear horizontal \\
\hline First side-lobe level & $-29 \mathrm{~dB}$ \\
\hline Steer ability & $360^{\circ}$ azimuth; $-1^{\circ}$ to $45^{\circ}$ elevation \\
\hline Rotation Rate & $30^{\circ} / \mathrm{s}$ (azimuth and elevation) \\
\hline Mechanical Limits & $-1^{\circ}$ to $60^{\circ}$ \\
\hline Angular Acceleration & $15^{\circ} s^{-2}$ (azimuth and elevation) \\
\hline Pointing Accuracy & $\pm 0.2^{\circ}$ \\
\hline \multicolumn{2}{|l|}{ Transmitter } \\
\hline Frequency Range & $2.7-3.0 \mathrm{GHz}$ \\
\hline Peak Power output & $500 \mathrm{~kW}$ into antenna \\
\hline \multirow[t]{2}{*}{ Pulse width } & 1.57 microseconds (short pulse) \\
\hline & 4.5 microseconds (long pulse) \\
\hline \multicolumn{2}{|l|}{$\mathrm{PRF}$} \\
\hline Long Pulse & $322-422 \mathrm{~Hz} \pm 1.7 \%$ \\
\hline Short Pulse & $322-1282 \mathrm{~Hz} \pm 1.7 \%$ \\
\hline
\end{tabular}

Table 2: Nexrad WSR Weather Radar Parameters 


\begin{tabular}{|l|l|c|}
\hline Band & Lower frequency $(\mathrm{MHz})$ & Upper frequency $(\mathrm{MHz})$ \\
\hline S-band & 2700 & 2900 \\
\hline C-band & 5250 & 5725 \\
\hline X-band & 9300 & 9500 \\
\hline
\end{tabular}

Table 3: Marine radar frequency band allocations [1].

\begin{tabular}{|l|l|l||l|l|l|l|}
\hline Property & $\begin{array}{l}2.9 \mathrm{GHz}- \\
3 \mathrm{GHz}\end{array}$ & & $\begin{array}{l}5.47 \mathrm{GHz}- \\
5.65 \mathrm{GHz}\end{array}$ & & $\begin{array}{l}8.85 \mathrm{GHz}^{-} \\
9 \mathrm{GHz}\end{array}$ & $\begin{array}{l}9.2 \mathrm{GHz}^{-} \\
9.5 \mathrm{GHz}\end{array}$ \\
\hline & Min. & Max. & Min. & Max. & Min. & Max. \\
\hline Antenna & & & & & & \\
\hline 3-dB Beamwidth (deg) & & & & & & \\
\hline Horizontal & 4.0 & 1.0 & 2.6 & 1.0 & 2.3 & 0.75 \\
\hline Vertical & 30.0 & 24.0 & 25.0 & 18.0 & 26.0 & 20.0 \\
\hline Sidelobe attenuation $(\mathrm{dB})$ & & & & & & \\
\hline Within $\pm 10^{\circ}$ & 28 & 23 & 29 & 23 & 31 & 23 \\
\hline Outside $\pm 10^{\circ}$ & 32 & 31 & 35 & 31 & 40 & 30 \\
\hline Gain $(\mathrm{dB}$ & 28 & 26 & 31 & 28 & 32 & 27 \\
\hline Rotation rate $(\mathrm{rpm})$ & 60 & 20 & 60 & 14 & 60 & 20 \\
\hline Transmitter & & & & & & \\
\hline Peak power $(\mathrm{kW})$ & 75 & 30 & 70 & 50 & 50 & 5 \\
\hline Frequency $(\mathrm{GHz})$ & 3.08 & 3.02 & 5.595 & 5.485 & $9.445 \pm 0.03$ & $9.375 \pm 0.03$ \\
\hline Pulse length $(\mathrm{micros})$ & 1.2 & 0.05 & 1.5 & 0.07 & 1.2 & 0.03 \\
\hline Receiver & & & & & & \\
\hline$($ IF) (MHz) & 60 & 45 & 60 & 45 & 60 & 45 \\
\hline IF bandwidth $(\mathrm{MHz})$ & & & & & & \\
\hline Short pulse $(\mu \mathrm{s})$ & 28 & 6 & 28 & 6 & 28 & 6 \\
\hline Medium/Long pulse $(\mu \mathrm{s})$ & 6 & 2.5 & 6 & 2.5 & 6 & 2.5 \\
\hline Noise figure $(\mathrm{dB})$ & 8.5 & 3 & 8.5 & 3 & 8.5 & 3.5 \\
\hline
\end{tabular}

Table 4: Typical characteristics of IMO category radio navigation radars. 


\begin{tabular}{|l|l|}
\hline Radar Parameter Feature & Value \\
\hline Antenna (Transmitter and Receiver) & \\
\hline Horizontal Beam width & $0.95^{\circ}$ \\
\hline Vertical Beam width & $26^{\circ}$ \\
\hline Side lobe attenuation & $>25 \mathrm{~dB}$ within $\pm 10^{\circ}$ of mainlobe \\
& $>32 \mathrm{~dB}$ outside $\pm 10^{\circ}$ of mainlobe \\
\hline Gain (dB) & 30 \\
\hline Antenna rotation speed & $30 \mathrm{rpm}$ \\
\hline Transmitter & \\
\hline Peak Output Power & $5 \mathrm{~kW}$ \\
\hline Frequency & $-9410 \mathrm{MHz} \pm 30 \mathrm{MHz}$ \\
\hline Pulse length (microseconds) & $0.05,0.18,0.5$ \\
\hline Pulse repetition rate(PRR)(Hz) & $1000 \mathrm{to} 3000$ \\
\hline Receiver & \\
\hline Intermediate frequency (IF) & $50 \mathrm{MHz}$ \\
\hline IF bandwidth & $15-25 \mathrm{MHz}$ \\
\hline Noise figure & $6 \mathrm{~dB}$ \\
\hline
\end{tabular}

Table 5: Typical characteristics of river class radio navigation radars. 


\begin{tabular}{|l|l|}
\hline Radar Parameter Feature & Value \\
\hline Antenna (Tx and Rx) & \\
\hline Horizontal Beam width & $0.95^{\circ}$ \\
\hline Vertical Beam width & $26^{\circ}$ \\
\hline Side lobe attenuation & $\begin{array}{l}>25 \mathrm{~dB} \text { within } \pm 10^{\circ} \text { of mainlobe } \\
\end{array}$ \\
\hline Gain (dB) & $30 \mathrm{~dB}$ outside $\pm 10^{\circ}$ of mainlobe \\
\hline Antenna rotation speed & $30 \mathrm{rpm}$ \\
\hline Transmitter & \\
\hline Peak Output Power & $5 \mathrm{~kW}$ \\
\hline Frequency & $-9410 \mathrm{MHz} \pm 30 \mathrm{MHz}$ \\
\hline Pulse length (microseconds) & $0.05,0.18,0.5$ \\
\hline Pulse repetition rate(PRR)(Hz) & $1000 \mathrm{to} 3000$ \\
\hline Receiver & \\
\hline Intermediate frequency (IF) & $50 \mathrm{MHz}$ \\
\hline IF bandwidth & $15-25 \mathrm{MHz}$ \\
\hline Noise figure & $6 \mathrm{~dB}$ \\
\hline
\end{tabular}

Table 6: Typical characteristics of pleasure-craft class radio navigation radars. 
is large in elevation (typically $18^{\circ}$ to $26^{\circ}$ ) and narrow in azimuth (typically $0.75^{\circ}$ to $4^{\circ}$ ). Azimuth beam widths in the vicinity of $1^{\circ}$ are common. Array-fed horn antennas are the most common antenna design and dual polarized $(\mathrm{H}$ and $\mathrm{V}$ linear polarizations) are becoming common for marine hazard detection, sea surface monitoring and meteorological observations.

In this thesis, we use the Furuno FR7111 X-band marine radar [73] as a baseline to construct a marine radar interference source model. The baseline system uses a rotating, two-dimensional slotted waveguide array, operates at frequencies in the vicinity of $9.4 \mathrm{GHz}$ and alternately transmits short and long pulses. Typical operating parameters [73] are displayed in Table 7. Sample marine radar chirp rates are noted in Table 8.

\subsection{Airborne radars}

Aircraft search radars are a primary air traffic control tools and can be grouped in three different classes: air route surveillance radars (ARSRs), airport surveillance radars (ASRs) and precision approach radars (PARs). The major properties of three ARSR and ASR systems in current use are shown in Table 9 [74].

ARSRs are long-range, three-dimensional surveillance radars designed to detect and track transiting aircraft within $400 \mathrm{~km}$ of the radar site. The most recent ARSR systems deployed around the continental US is the ARSR-4 pulse-compression radar class. ARSR-4 is an L-band radar operating in the 1.2 to $1.4 \mathrm{GHz}$ frequency range. Radio spectrum reallocation proposals to free more bandwidth for commercial communications would reduce the upper bound of the frequency range to $1390 \mathrm{GHz}$. Since the ARSR-4 system uses frequency hopping within its operation band, 4 of the 22 frequency band pairs would be lost.

ASRs are intermediate range radars found at all major airports. These are Sband instruments operating between $2.7 \mathrm{GHz}$ and $2.9 \mathrm{GHz}$ with peak radiated power near 1.1 MW for the older, short-pulse ASR-9 class radars still in wide use in North America and $25 \mathrm{~kW}$ for the current pulse-compression ASR-11 class radars. ASRs are designed to detect and track aircraft with radar cross section greater than 1 metre squared at altitudes up to $19 \mathrm{~km}$ and high-altitude radar ranges up to $105 \mathrm{~km}$. 


\begin{tabular}{|l|l|}
\hline Radar Parameter Feature & Value \\
\hline Radiator & Slotted waveguide array \\
\hline Polarisation & Linear horizontal \\
\hline Antenna rotation speed & $24 \mathrm{rpm}$ \\
\hline Radiator length (XN13A) & $180 \mathrm{~cm}$ \\
\hline Horizontal Beam width (XN13A) & $1.2^{\circ}$ \\
\hline Vertical Beam width & $22^{\circ}$ \\
\hline Side lobe attenuation(XN13A) & Within $\pm 10^{\circ}$ of main-lobe: $<-24 \mathrm{~dB}$ \\
\hline Transceiver module & Outside $\pm 10^{\circ}$ of main-lobe: $<-30 \mathrm{~dB}$ \\
\hline Frequency & $-9410 \mathrm{MHz} \pm 30 \mathrm{MHz}$ \\
\hline Modulation & Continuous wave \\
\hline Peak Output Power & $10 \mathrm{~kW}$ \\
\hline Pulse length $(\tau)$ and & At $0.125 \mathrm{~nm}-0.75 \mathrm{~nm}, \tau=0.12 \mu \mathrm{s}$, \\
Pulse Repetition rate(PRR) & PRR $=2100 \mathrm{~Hz}$ \\
\hline & $\begin{array}{l}\text { At } 1-2 \mathrm{~nm}, \tau=0.3 \mu \mathrm{s} \\
\text { PRR }=1200 \mathrm{~Hz}\end{array}$ \\
\hline Intermediate frequency $(\mathrm{IF})$ & At $3-24 \mathrm{~nm}, \tau=0.8 \mathrm{micros}, \mathrm{PRR}=600 \mathrm{~Hz}$ \\
IF bandwidth & $25 \mathrm{MHz}$ \\
\hline \hline
\end{tabular}

Table 7: Furono FR7111 Marine Radar Parameters 


\begin{tabular}{|l|l|l||l||}
\hline $\begin{array}{l}\text { Centre } \\
\text { frequency } \\
(\text { MHZ })\end{array}$ & $\begin{array}{l}\text { Pulse } \\
\text { width }(\mu \mathrm{s})\end{array}$ & PRF $(\mathrm{MHz})$ & BW $(\mathrm{MHz})$ \\
\hline Radar 1 & & 2100 & 25 \\
\hline 9400 & 0.12 & 1200 & 25 \\
\hline Radar 2 & & & \\
\hline 9400 & 0.3 & 600 & 25 \\
\hline Radar 3 & & 0.8 & \\
\hline 9400 &
\end{tabular}

Table 8: Sample marine radar chirp rates.

\begin{tabular}{|c|c|c|c|c|c|}
\hline Function & Sensitivity & Range/Alt & $\mathrm{Az} / \mathrm{El}$ & Waveform & $\begin{array}{l}\text { Update } \\
\text { rate }\end{array}$ \\
\hline $\begin{array}{l}\text { Terminal } \\
\text { aircraft } \\
\text { surveil- } \\
\text { lance }\end{array}$ & $1 \mathrm{~m}^{2}$ & $\begin{array}{l}60 \mathrm{~nm} / 20,000 \\
\mathrm{ft}\end{array}$ & $1.4^{\circ} / 5^{\circ}$ & $\begin{array}{l}>18 \\
\text { pulses;PRI } \\
1 \mathrm{~ms}\end{array}$ & $5 \mathrm{~s}$ \\
\hline $\begin{array}{l}\text { En Route } \\
\text { aircraft } \\
\text { surveil- } \\
\text { lance }\end{array}$ & $2.2 \mathrm{~m}^{2}$ & $\begin{array}{l}250 \\
\mathrm{~nm} / 60,000 \mathrm{ft}\end{array}$ & $1.4^{\circ} / 2^{\circ}$ & $\begin{array}{l}>10 \\
\text { pulses;PRI } \\
3 \mathrm{~ms}\end{array}$ & $12 \mathrm{~s}$ \\
\hline $\begin{array}{l}\text { Terminal } \\
\text { area } \\
\text { weather }\end{array}$ & $\begin{array}{l}-20 \text { to } 5 \\
\mathrm{dBz}\end{array}$ & $\begin{array}{l}600 \\
\mathrm{~nm} / 15,000 \mathrm{ft}\end{array}$ & $1^{\circ} / 1^{\circ}$ & $\begin{array}{l}>50 \\
\text { pulses;PRI } \\
1 \mathrm{~ms}\end{array}$ & $60 \mathrm{~s}$ \\
\hline $\begin{array}{l}\text { National } \\
\text { scale } \\
\text { weather }\end{array}$ & $\begin{array}{lll}-20 & \text { to } \quad 5 \\
\mathrm{dBz} & \end{array}$ & $\begin{array}{l}250 \\
\mathrm{~nm} / 60,000 \mathrm{ft}\end{array}$ & $1^{\circ} / 1^{\circ}$ & $\begin{array}{l}>50 \\
\text { pulses;PRI } \\
1 \mathrm{~ms}\end{array}$ & $300 \mathrm{~s}$ \\
\hline
\end{tabular}

Table 9: Air radar pulse transmission schemes by function. 
Recent designs of ARSR and ASR civilian and military radar use compressed pulse excitation to increase peak pulse power by increasing the radiated pulse length. Recent military air surveillance radars use solid-state active array technology with two-dimensional electronic scan (often combined with a mechanical azimuthal scan) to maximize radar flexibility and maximize mean time between failures. Many systems use non-linear FM coding [75] and [76] in the expanded pulse to minimize range compression side lobes.

Many ASR and ARSR systems use a frequency diversity strategy of transmitted pulse generation in which radar pulses are transmitted simultaneously at two frequencies (for the ASR4 these are $83 \mathrm{MHz}$ apart and are stepped to create several transmit pulse frequency pairs) to minimize the effects of scattering nulls on radar target detection. The transmitted frequency plan is programmable and proprietary for each radar type. To maximize radar blind speed for target motion measurements, pulse transmissions are sequentially generated with different PRIs selected by proprietary protocols to yield a maximum-range mean PRF.

Precision approach radars (PARs) operate in the 9.1 to $9.2 \mathrm{GHz}$ Band. PARs are designed to provide aircraft glide slope data out to a maximum range of $40 \mathrm{~km}$. Depending on the radar design, some PARs use two fan beam antennas, one for elevation (narrow beam width $0.75^{\circ}$ ) and one for azimuth (narrow beam width $1.45^{\circ}$ ) and others use electronically scanned pencil beams in one or both dimensions. PARs provide an azimuth sector scan between $20^{\circ}$ and $30^{\circ}$ and an elevation sector scan between $8^{\circ}$ and $15^{\circ}$ good For applications where multiple glide-path azimuths are needed, the antenna beams are mechanically pointed in azimuth to select the glidepath to be monitored. Real-pulse radars transmit peak power between $180 \mathrm{~kW}$ and $300 \mathrm{~kW}$ with pulse bandwidths between $4 \mathrm{MHz}$ and $0.5 \mathrm{MHz}$. Compressed-pulse PARs can achieve effective performance for peak pulse power less than $20 \mathrm{~kW}$. Radar pulse repetition rates very between $3400 \mathrm{~Hz}$ and $3800 \mathrm{~Hz}$. Most PARs use two simultaneously transmitted frequencies and frequency hopping to minimize the effects of target response nulls.

Air surveillance radars operate at $\mathrm{S}$ band frequencies as shown in Table 6. The long-range systems used to support airport surveillance operate in the L-band between 1.2 and $1.4 \mathrm{GHz}$, while the airport surveillance S-band radars typically operate from 2.7 to $2.9 \mathrm{GHZ}$ to support local sites.

In this thesis, we will use the ASR-9 airport surveillance S-band system as a 


\begin{tabular}{|l|l|}
\hline Parameter & Value \\
\hline Radiator & $\begin{array}{l}\text { Parabolic dish antenna } \\
\text { offset-feed with a main Tx/Rx feed horn } \\
\text { passive Rx only feed horn }\end{array}$ \\
\hline Polarisation & Selectable circular or linear \\
\hline Antenna rotation speed & $12.5 \mathrm{rpm}$ \\
\hline Azimuth Beam width & $1.3^{\circ}$ \\
\hline Elevation Beam width & $4.8^{\circ}$ \\
\hline Gain & $33.5 \mathrm{~dB}($ Main beam LOW) to 32.5 dB (Main beam HIGH) \\
\hline Side lobe attenuation & $24 \mathrm{~dB}($ Azimuth); $6 \mathrm{~dB}$ (Elevation) \\
\hline Frequency & $2700-2900 \mathrm{~Hz}$ \\
\hline Peak Output Power & $1.32 \mathrm{MW}$ \\
\hline PRF & $1200 \mathrm{~Hz}$ \\
\hline Pulse width & $1.05 \mathrm{microseconds}$ \\
\hline
\end{tabular}

Table 10: ASR-9 airborne radar parameters.

baseline airport surveillance radar interference source. The baseline system uses a rotating, circular, offset-fed parabolic dish antenna, operates at frequencies in the vicinity of 2.7 to $2.9 \mathrm{GHz}$ and alternately transmits long and short CW pulses. Typical operating parameters are displayed in Table 10.

Typical airborne radar chirp rates are defined in Table 11. The magnitude of the received signal is determined via the radar range equation.

\subsection{Summary}

This chapter provided an overview of the various radars considered in this thesis. The three radars, namely the weather, the marine and the airborne radars can operate in several modes. The realistic chirp rates for weather, marine, and airborne radars have been defined based on the parameters contained in Sections 4.2, 4.3, and 4.4. Table 12 provides a summary. These typical chirp rates are considered as the known 


\begin{tabular}{|l|l|}
\hline Parameter & Value \\
\hline Radar 1 & \\
Centre Frequency (MHz) & 9750 \\
\hline Transmit bandwidth (MHz) & 50 \\
\hline Transmit pulse length (microseconds) & 50 \\
\hline Transmit PRF (Hz) & 2000 \\
\hline Rx Complex Sampling Rate (MHz) & 50 \\
\hline Radar 2 & \\
Centre Frequency (MHz) & 9750 \\
\hline Transmit bandwidth (MHz) & 1500 \\
\hline Transmit pulse length (microseconds) & 50 \\
\hline Transmit PRF (Hz) & 1000 \\
\hline Rx Complex Sampling Rate (MHz) & 200 \\
\hline \hline
\end{tabular}

Table 11: Sample airborne radar chirp rates.

set of chirp rates in this study. As seen from Table 12, some of the radars have the same centre frequency but may differ in their bandwidths. In a low SNR condition, it may be rather impossible to estimate the bandwidth. Resorting to machine learning techniques to first determine the type of radar and possibly its mode, appears as a logical approach. The following chapter will provide further insight into the simulations done to explore the idea of using machine learning with matched filter outputs to classify these radars. 


\begin{tabular}{|c|c|c|c|c|c|c|c|c|c|c|c|c|c|c|c|}
\hline 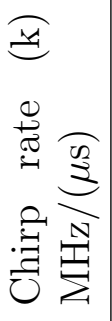 & $\neg$ & ని & $\underset{\Xi}{\leftrightarrows}$ & $\stackrel{0}{\stackrel{1}{0}}$ & $\underset{\infty}{\infty}$ & $\ddot{m}$ & 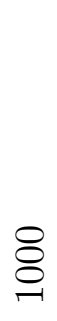 & $\begin{array}{l}\stackrel{10}{N} \\
\infty\end{array}$ & ஓి & 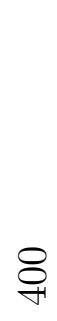 & $\underset{\exists}{\beth}$ & $\stackrel{9}{\not}$ & $\stackrel{8}{8}$ & $\stackrel{\text { ले }}{\sim}$ & $\stackrel{1}{10}$ \\
\hline 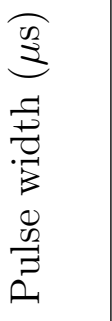 & 오 & 오 & $\stackrel{F}{*}$ & $\vec{N}$ & مْ ْمَ & مَ & $\stackrel{\bullet}{0}$ & $\stackrel{0}{-}$ & $\underset{\sim}{\stackrel{\circ}{0}}$ & $\stackrel{10}{0}$ & $\stackrel{\infty}{\stackrel{\infty}{0}}$ & $\stackrel{100}{0}$ & $\stackrel{10}{\varrho}$ & $\stackrel{\infty}{\stackrel{\infty}{0}}$ & $\stackrel{10}{0}$ \\
\hline 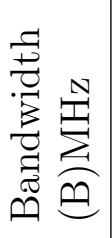 & 오 & $\underset{\stackrel{1}{8}}{\stackrel{8}{1}}$ & $\stackrel{8}{8}$ & \begin{tabular}{l}
$\qquad 0$ \\
\hdashline \\
$ن$
\end{tabular} & 8 & 8 & 8 & 8 & 8 & $\stackrel{\sim}{\sim}$ & $\stackrel{\curvearrowright}{\sim}$ & จ & $\stackrel{10}{\sim}$ & $\stackrel{10}{\sim}$ & $\stackrel{10}{N}$ \\
\hline 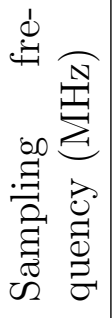 & $\stackrel{\circ}{\circ}$ & $\underset{\overbrace{}}{\stackrel{\overbrace{}}{\circ}}$ & 오 & 오 & 요 & 요 & 요 & 오 & 요 & 요 & 요 & 누 & 옹 & 오 & 요 \\
\hline 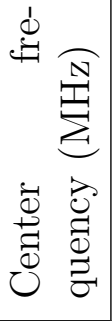 & $\begin{array}{l}\stackrel{0}{10} \\
\stackrel{1}{\infty} \\
\sigma\end{array}$ & $\begin{array}{l}\stackrel{0}{10} \\
\stackrel{1}{5}\end{array}$ & $\begin{array}{l}\stackrel{0}{10} \\
\stackrel{1}{5}\end{array}$ & $\begin{array}{l}\stackrel{0}{10} \\
\stackrel{1}{\circ}\end{array}$ & $\begin{array}{l}8 \\
\stackrel{1}{10} \\
0\end{array}$ & $\begin{array}{l}8 \\
8 \\
20 \\
0\end{array}$ & $\begin{array}{l}8 \\
8 \\
20 \\
0\end{array}$ & 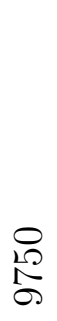 & $\begin{array}{l}\stackrel{1}{10} \\
\stackrel{1}{0}\end{array}$ & $\begin{array}{l}\stackrel{\varrho}{\exists} \\
\stackrel{+}{\not H}\end{array}$ & $\begin{array}{l}\stackrel{\varrho}{\exists} \\
\stackrel{+}{\sigma}\end{array}$ & $\begin{array}{l}\stackrel{0}{\not} \\
\stackrel{F}{\sigma}\end{array}$ & $\begin{array}{l}\stackrel{0}{\rightrightarrows} \\
\stackrel{\not}{\sigma}\end{array}$ & $\begin{array}{l}\stackrel{\bigcirc}{\rightrightarrows} \\
\stackrel{\rightrightarrows}{\sigma}\end{array}$ & $\stackrel{\circ}{\stackrel{ }{\sharp}}$ \\
\hline 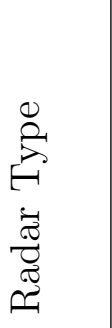 & 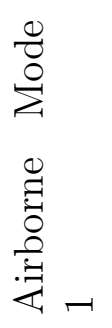 & 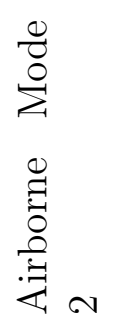 & 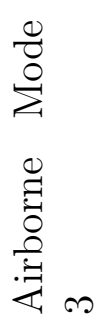 & 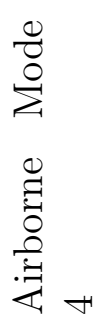 & 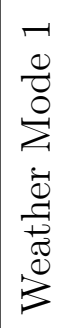 & 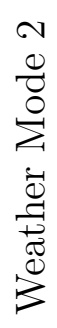 & 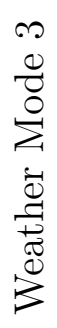 & 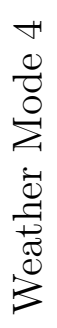 & 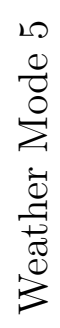 & 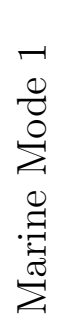 & 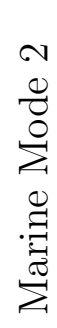 & 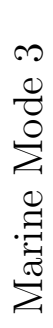 & 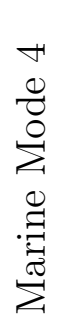 & 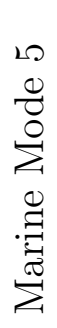 & 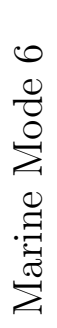 \\
\hline
\end{tabular}




\section{Chapter 5}

\section{Results}

\subsection{Introduction}

In this chapter, the inadequacies of the Wigner-Ville transform and the peak detection using a bank of matched filters are presented as the motivation for this thesis work. A bank of $M$ matched filters whose individual filter impulse response correspond to clean ideal LFM radar waveform for the chirp rate contained in the known set is constructed and used in this work. Different simulation scenarios are discussed and the results are analyzed.

\subsection{Inadequacy of the Wigner-Ville Transform}

The Wigner-Ville transform as described in Chapter 3 was applied on a LFM pulse with $\beta=1$. Figure 3 shows the Wigner-Ville Transform of a LFM signal in the absence of noise. The Wigner-Ville transform for the SNR of $0 \mathrm{~dB}$ is shown in Figure 4. The line is still somewhat visible. When the SNR is-10 dB and below, the line is no longer visible as seen in Figures 5 and 6. This motivates consideration of other approaches to detecting LFM signals particularly in low-SNR conditions.

\subsection{Proposed approach}

Normally as a first stage in the detection of signals in a radar receiver, matched filtering is carried out. However, in the radar intercept receiver, we do not have the 


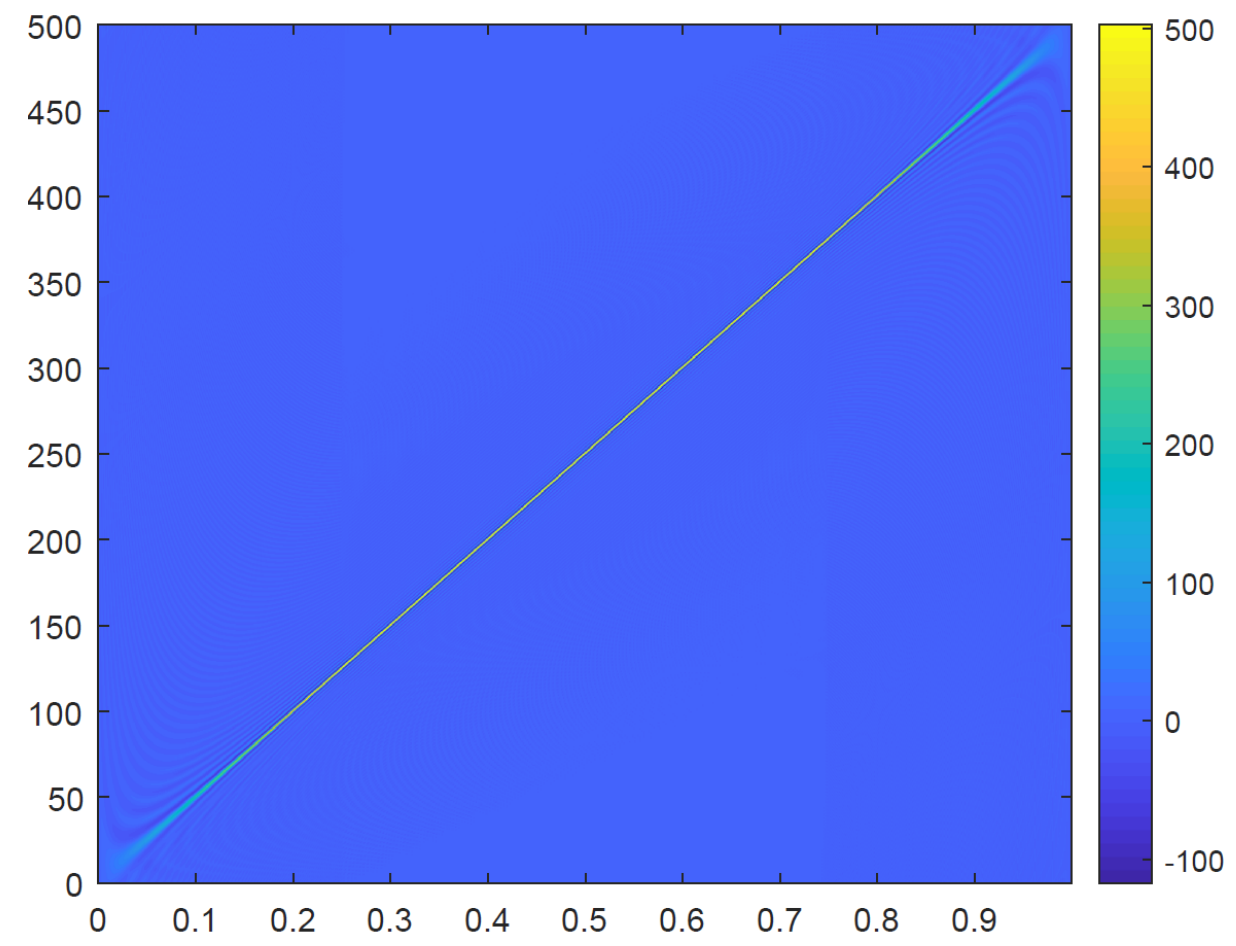

Figure 3: Wigner-Ville transform of an LFM signal.

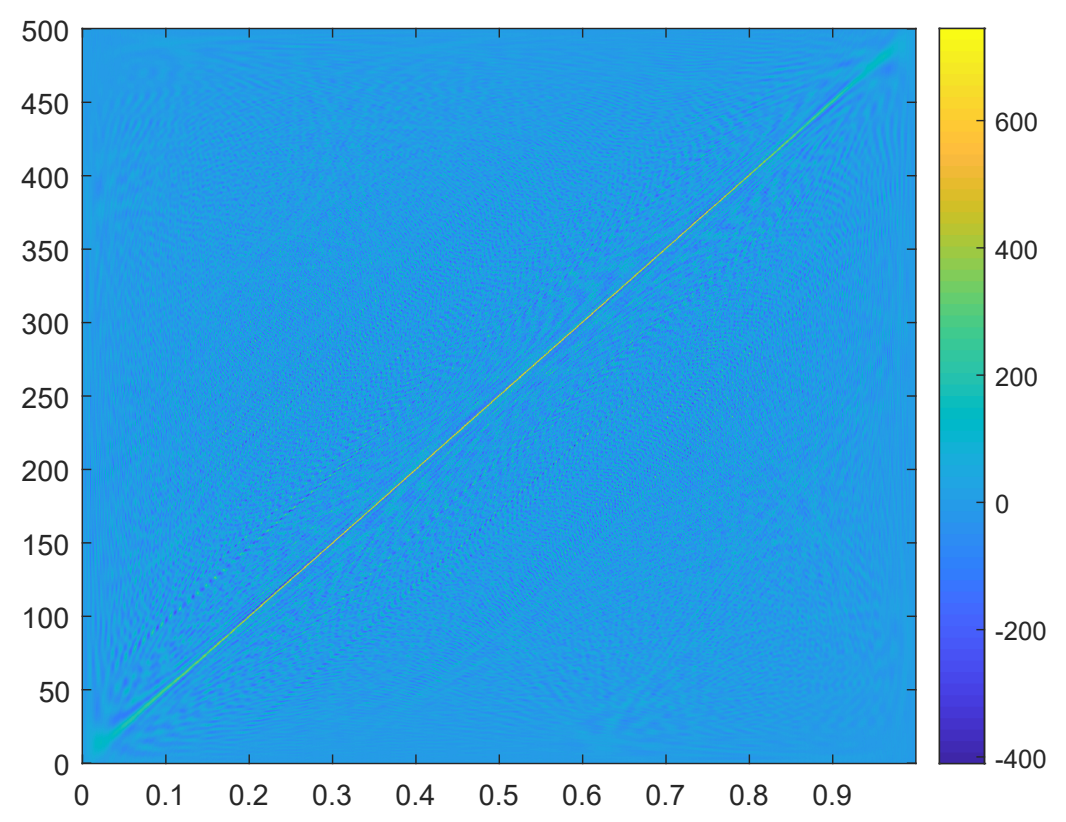

Figure 4: Wigner-Ville transform of a noisy LFM signal at a SNR of $0 \mathrm{~dB}$. 


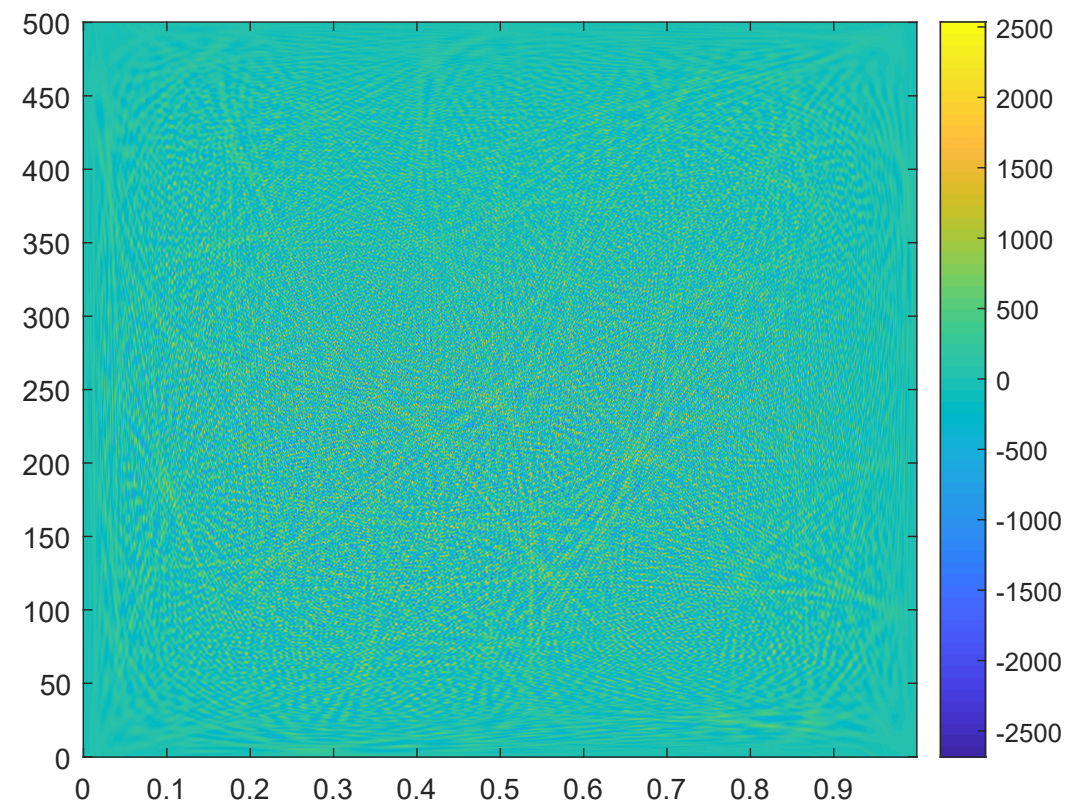

Fioure 5: Wioner-Ville transform of an T.FM sional a.t a. SNR of $-10 \mathrm{~dB}$.

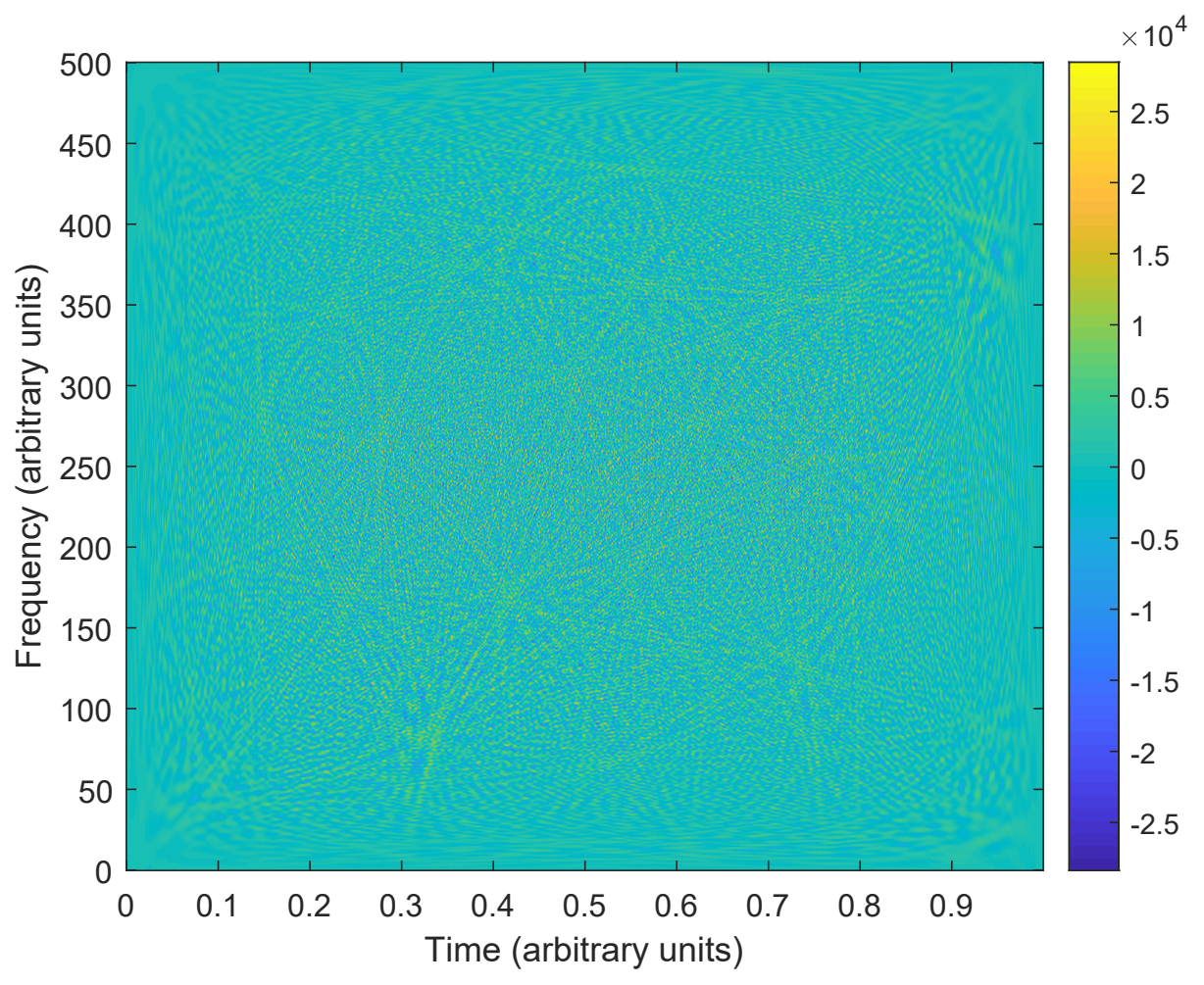

Figure 6: Wigner-Ville transform of a noisy LFM signal at a SNR of $-20 \mathrm{~dB}$. 
information about the transmitted waveform (chirp rate in the case of LFM signals). Therefore, the traditional radar approach will not be successful in determining the chirp rate.

The approach we will explore for determining $\beta$ is to perform matched filtering of an incoming signal with an unknown chirp rate, which is denoted simply as $x[n]$, against all the ideal signals $x_{\beta_{1}}[n], \ldots, x_{\beta_{m}}[n]$. The outputs of these matched filters are concatenated to form the input which is then fed into a machine learning classifier. The classifier outputs a prediction of the chirp rate of the signal.

In the case of additive white noise as assumed above, the matched filter $h_{\beta}[n]$ corresponding to a LFM signal $x_{\beta}[n]$ is:

$$
h_{\beta}[n]=\frac{x_{\beta}^{*}[-n]}{\sum_{k=0}^{N-1} x_{\beta}[k] x_{\beta}^{*}[k]}
$$

where the superscript $*$ indicates complex conjugation and the denominator is simply a normalization factor. The output of the matched filter is simply the convolution $x[n] * h_{\beta}[n]$. This amounts, in fact, to calculating the cross-correlation between the signals $x[n]$ and $x_{\beta}[n]$, so we expect a large output when the incoming signal is similar to the ideal LFM signal $x_{\beta}[n]$ and a small output when the two signals are very different. In the following, we will assume that the input $x[n]$ is normalized just like the filter $h_{\beta}[n]$ is. In effect, we calculate the normalized cross-correlation instead of the cross-covariance.

Because we know the set that $\beta$ is drawn from, we can perform matched filtering of the incoming signal against all of the signals $x_{\beta_{1}}[n], \ldots, x_{\beta_{m}}[n]$. Heuristically speaking, we would expect that the matched filter output with the highest peak will tell us which of the chirp rates best describes the incoming signal. However, at low-SNR, this simple peak detection approach may not be robust. In order to develop a more robust algorithm, we propose a machine learning approach to this problem using the concatenated matched filter outputs.

The machine learning portion of our procedure involves concatenating all of the matched filter outputs $x[n] * h_{\beta_{1}}[n], \ldots, x[n] * h_{\beta_{m}}[n]$ into one long vector. This vector is then considered as a feature vector to be fed into a classifier. An example of such a feature vector, assuming no noise, is shown in Fig. 7. By training a classifier on a large number of matched filter outputs for various chirp rates and signal-to-noise 


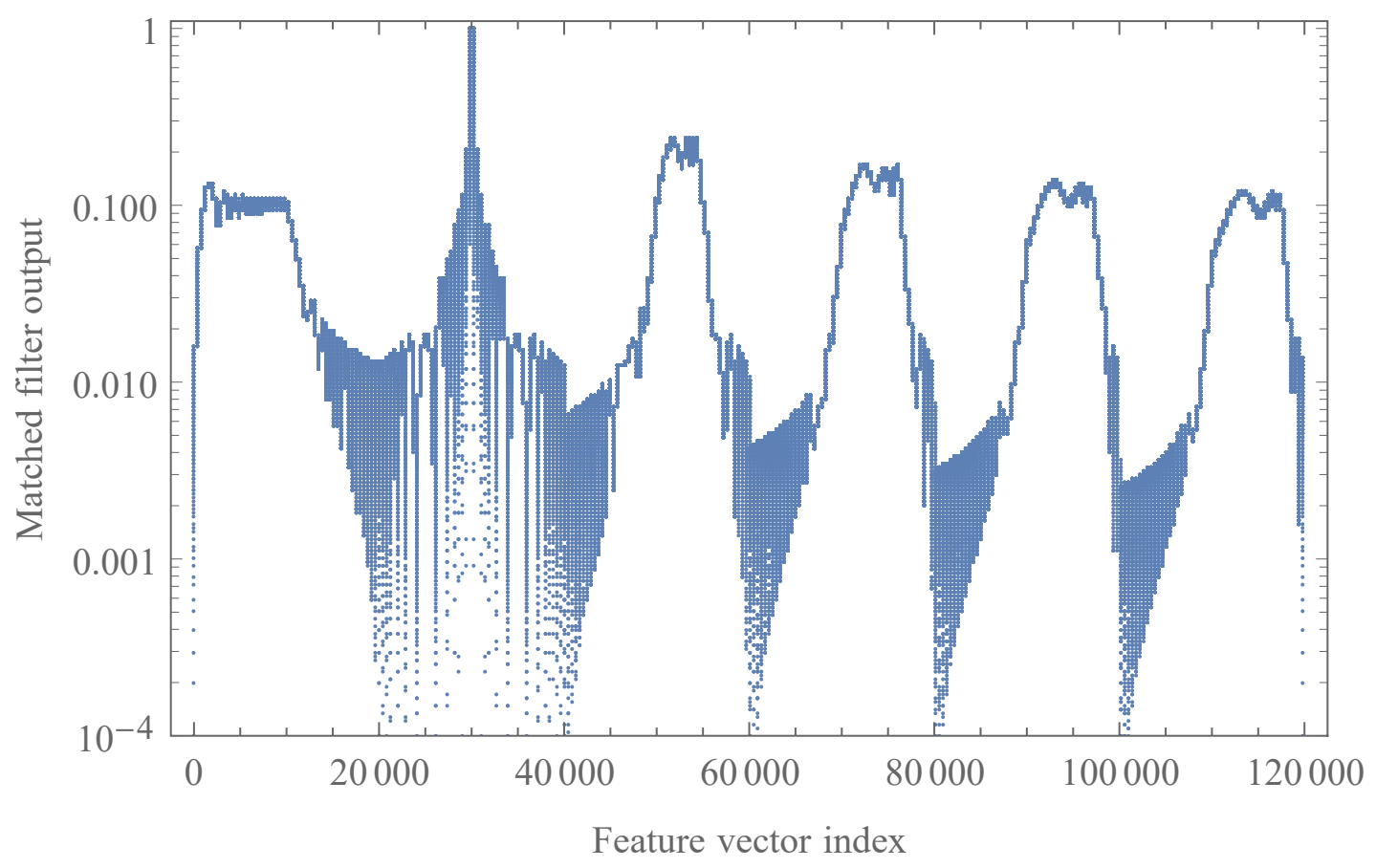

Figure 7: Example of a feature vector obtained by concatenating all the matched filter outputs of an ideal chirp.

ratios (SNRs), we expect to obtain a classifier which will accurately predict the chirp rate of the incoming signal.

Four machine learning algorithms, namely the Decision Tree, the Naive Bayes, the Random Forest, and the Support Vector Machine (SVM) are used to classify the chirp parameter.

One might ask why not simply use the peak of the match filter output to determine the chirp rate. Figure 8 shows the example of a case where no peak is evident. We shall see later that the signal is correctly classified correctly using the proposed ML approach.

\subsection{Classifiers Evaluated in This Thesis}

There are seven distinct classification problems for which ML classifiers were trained:

- Problem 1: This may be used as demonstration of the proof-of-concept through a toy problem. The intent here is to classify LFM signals whose center frequencies are all $0 \mathrm{~Hz}$ and whose chirp rates are taken from the set $\beta=0,1,2,3,4$, 


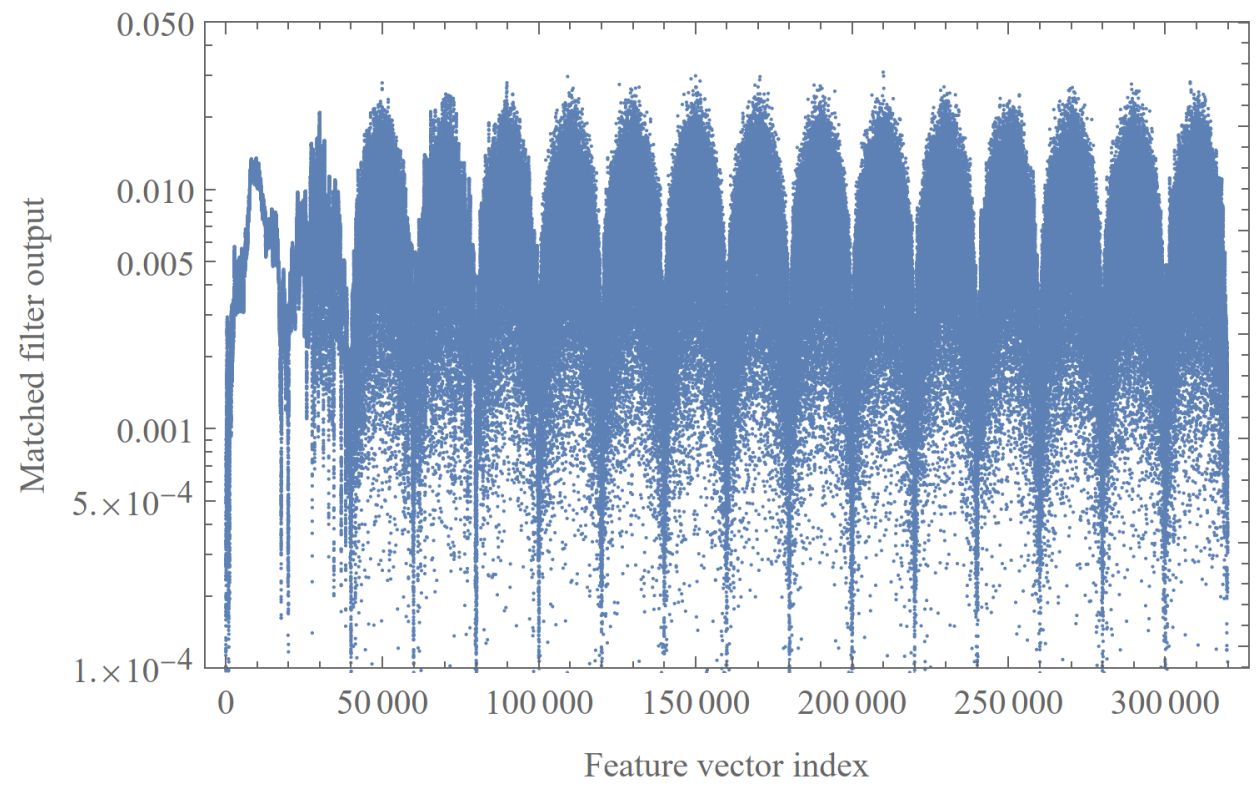

Figure 8: Concatenated match filter approach applied to LFM signal at $-10 \mathrm{~dB}$. No clear peak is evident making it difficult to accurately classify the chirp rate.

and $5 \mathrm{~Hz} / \mathrm{s}$ (6-class problem).

- Problem 2: This problem considers realistic chirp rates of only airborne radars: The intent here is to classify LFM signals with realistic parameters corresponding to airborne radars as listed in Table 12 (4 modes + no chirp $=5$-class problem).

- Problem 3: This problem considers realistic chirp rates of only weather radars. The intent here is to classify LFM signals with parameters corresponding to weather radars as listed in Table 12 ( 5 modes + no chirp $=6$-class problem $)$.

- Problem 4: This problem considers realistic chirp rates of only marine radars. The intent here is to classify LFM signals with parameters corresponding to marine radars as listed in Table 12 (6 modes + no chirp $=7$-class problem $)$.

- $\underline{\text { Problem 5: }}$ The intent here is to consider realistic chirp rates of all radar types considered in this research. The intent here is to classify LFM signals with parameters corresponding to all the radars listed in Table 12 (15 modes + no chirp $=16$-class problem).

- Problem 6: The intent here is to study the robustness of the classifiers against 
windowing imperfections. This can occur if the intercept receiver does not turn on immediately when the radar transmitter's pulse is received. As a consequence, some part of the pulse is lost. The classifiers trained in Problems 1 and 5 are used for this robustness study.

- Problem 7: The intent here is to study the robustness of the classifier against perturbation in chirp parameters. The classifier trained for Problem 5 is used in this study. In practice, this situation arises due to hardware imperfections.

\subsubsection{Details of Simulations}

For each of Problems 1-5 listed above, four machine learning algorithms were used: decision tree (DT), naive Bayes (NB), random forest (RF), and support vector machine (SVM). There are two exceptions: for Problem 1, as it was a proof-of-concept study, we tried only NB and SVM classifiers. All classifiers were trained using Wolfram Mathematica's Classify command, which is a high-level command that automatically optimizes each classifier without human input (e.g., by choosing hyperparameters and selecting appropriate kernels). No classifiers were trained for problems 6 and 7; they used the ones trained for Problem 5.

For Problem 1, LFM signals were simulated with a sampling frequency of $f_{s}=$ $2000 \mathrm{~Hz}$; the number of samples in each sampled signal was $N=10000$. Each signal was contaminated with additive white Gaussian noise, resulting in simulated signals with SNRs of 20, 10, 0, $-10,-20,-30$, and $-40 \mathrm{~dB}$. The training set consisted of 200 noisy LFM signals for each $\beta$ and each SNR, for a total of 8400 signals. The trained classifiers were then tested on a test set containing $50 \mathrm{LFM}$ signals for each $\beta$ and SNR (for a total of 2100 signals), and confusion matrices were generated separately for each SNR.

For Problems 2 to Problem 5, LFM signals were simulated with a sampling frequency of $f_{s}=1200 \mathrm{MHz}$, though the number of samples in each sampled signal remained at $N=10000$. Each signal was contaminated with additive white Gaussian noise, resulting in simulated signals with SNRs of 10, 0, -5, -10, -15, and $-20 \mathrm{~dB}$. The training set consisted of 50 noisy LFM signals for each set of parameters and each SNR. Thus, for Problem 2, the training set contained 1500 samples, while the training sets for Problem 3 to Problem 5 contained 1800, 2100, and 4000 samples respectively. The test set contained 10 LFM signals for each $\beta$ and SNR, 
so the size of the test sets for Problems 2-5 were 250, 300, 350, and 800 samples respectively. In all cases, confusion matrices were generated separately for each SNR.

In Problem 6, the classifiers for Problem 1 and Problem 5 were subjected to a further test to understand the effect of imperfect windowing. In this test, the last $20 \%$ of each chirp were dropped, and a corresponding number of zeros were added to the beginning of each chirp in order to pad them back to the original length. An example of this procedure is shown in 36. This was performed for each of the training sets described above for Problems 1 and 5. Note that the noise was added after the chirp was "shifted", so the first $20 \%$ of each noisy chirp corresponds to pure noise.

Finally, in Problem 7 the classifiers for Problem 5 (which were trained on all the radars in Table 12) were subjected to yet another test in order to characterize the robustness of the approach considered in this thesis. This time, the classifiers were presented with simulated LFM signals whose parameters were not drawn from Table 12. A list of five such "unknown" radars is given in Table 13. For each entry in this list, the type of radar is given, as well as the radar in Table 12 whose parameters come closest to each unknown radar. The classifiers cannot possibly classify these radars correctly, as the radar parameters do not fall under any of the classes which the classifiers are designed to classify. It is hoped, however, that the classifier returns the radar in Table 12 which comes closest to a given unknown radar, or at least returns a radar whose type is the same as the unknown radar's type. If this is successful, then the same classifiers used to solve Problem 5 could also be used to classify the type (but not the mode) of radars whose LFM parameters are unknown to the classifiers. For this test, the same sampling frequency, signal length, and SNRs that were listed above for Problems 2-5 were used, with 10 samples for each unknown radar and SNR (300 samples total).

\subsection{Results of Simulations}

\subsubsection{Problem 1: LFM signals with pre-selected chirp rates}

Naive Bayes and SVM classifiers classified the test set perfectly for all signals with an SNR of $-10 \mathrm{~dB}$ or above. Therefore, classification above $-10 \mathrm{~dB}$ is not included in the thesis. Performance begins to degrade at $-20 \mathrm{~dB}$, though at this level of 


\begin{tabular}{|c|c|c|c|c|}
\hline \hline Label & Radar Type & Center freq $(\mathrm{MHz})$ & $\beta \mathrm{MHz} /(\mu \mathrm{s})$ & Similar to Radar \\
\hline Radar A & Marine & 9410 & 42 & Marine Mode 3 \\
\hline Radar B & Weather & 9500 & 373 & Weather Mode 4 \\
\hline Radar C & Air & 9750 & 15 & Air Mode 3 \\
\hline Radar D & Marine & 9410 & 128 & Marine Mode 5 \\
\hline Radar E & Air & 9750 & 31 & Air Mode 2 \\
\hline \hline
\end{tabular}

Table 13: Radar types and signal parameters for a set of radars that are unknown to the bank of matched filters.

noise the performance is still quite good, as can be seen in Figs. 9 and 10. However, performance at $-30 \mathrm{~dB}$ and below was poor. In summary, good classification performance was achievable down to an SNR of $-20 \mathrm{~dB}$ even with the simple, unsophisticated approach taken here. This is significantly better than what is possible with the Wigner-Ville transform.

\subsubsection{Problem 2: Airborne radar realistic chirp results}

The confusion matrix for a decision tree classifier of realistic airborne radar chirp rates is shown in Figure 11. DT makes classification errors at $\mathrm{SNR}=-15 \mathrm{~dB}$ with misclassification of mode 2 as mode 4 . At $-20 \mathrm{~dB}$, the only modes that have no issues are no radar and mode 4 . The chirp rate that is the hardest to detect at this SNR level is mode 3 .

The confusion matrix result for a naive Bayes classifier of realistic chirp rates for airborne radars are shown in Figure 12 for the following SNR values $10 \mathrm{~dB}, 0 \mathrm{~dB}$, $-5 \mathrm{~dB},-10 \mathrm{~dB},-15 \mathrm{~dB}$ and $-20 \mathrm{~dB}$. From the confusion matrix, it is clear that this classifier has performance degradation with decreasing SNR. At -20 dB, this classifier fails.

The confusion matrix result for a Random Forest classifier of realistic chirp rates for airborne radars are shown in Figure 13 for the following SNR values $10 \mathrm{~dB}, 0 \mathrm{~dB}$, $-5 \mathrm{~dB},-10 \mathrm{~dB},-15 \mathrm{~dB}$ and $-20 \mathrm{~dB}$. RnF performs well till $0 \mathrm{~dB}$ and the performance deteriorates very rapidly as the SNR worsens.

The confusion matrix result for a Support Vector Machine (SVM) classifier of 


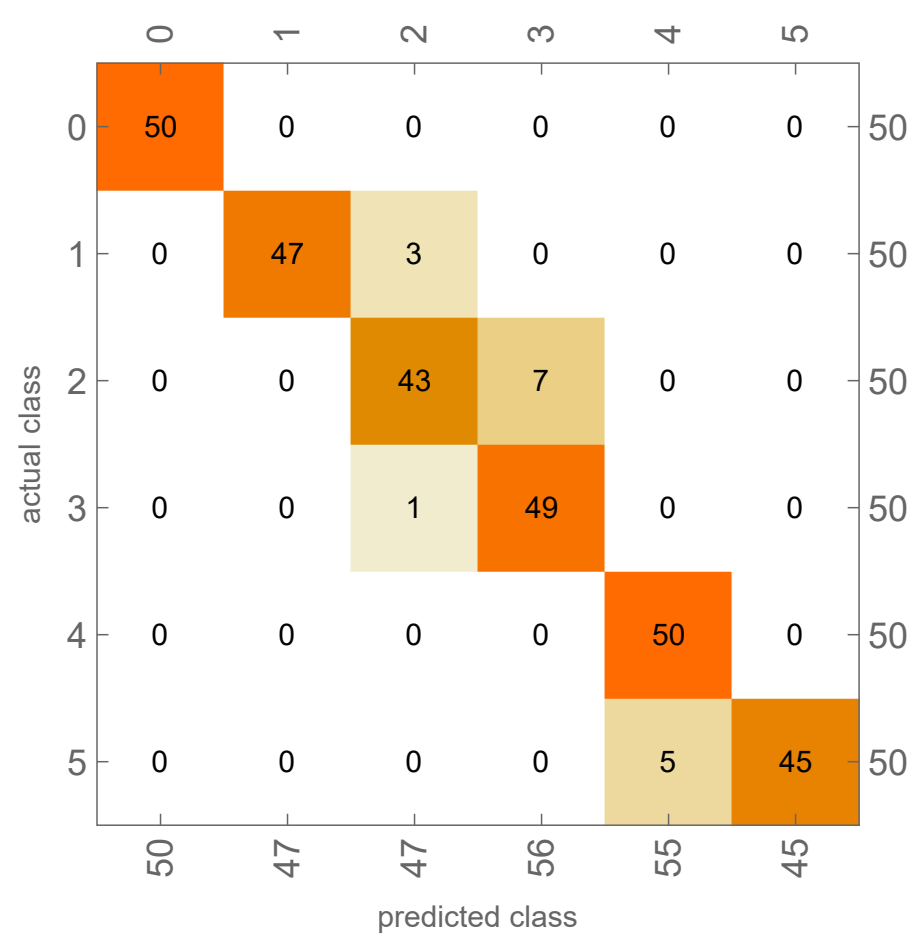

Figure 9: Confusion matrix for a NB classifier at $-20 \mathrm{~dB}$ SNR (Problem 1).

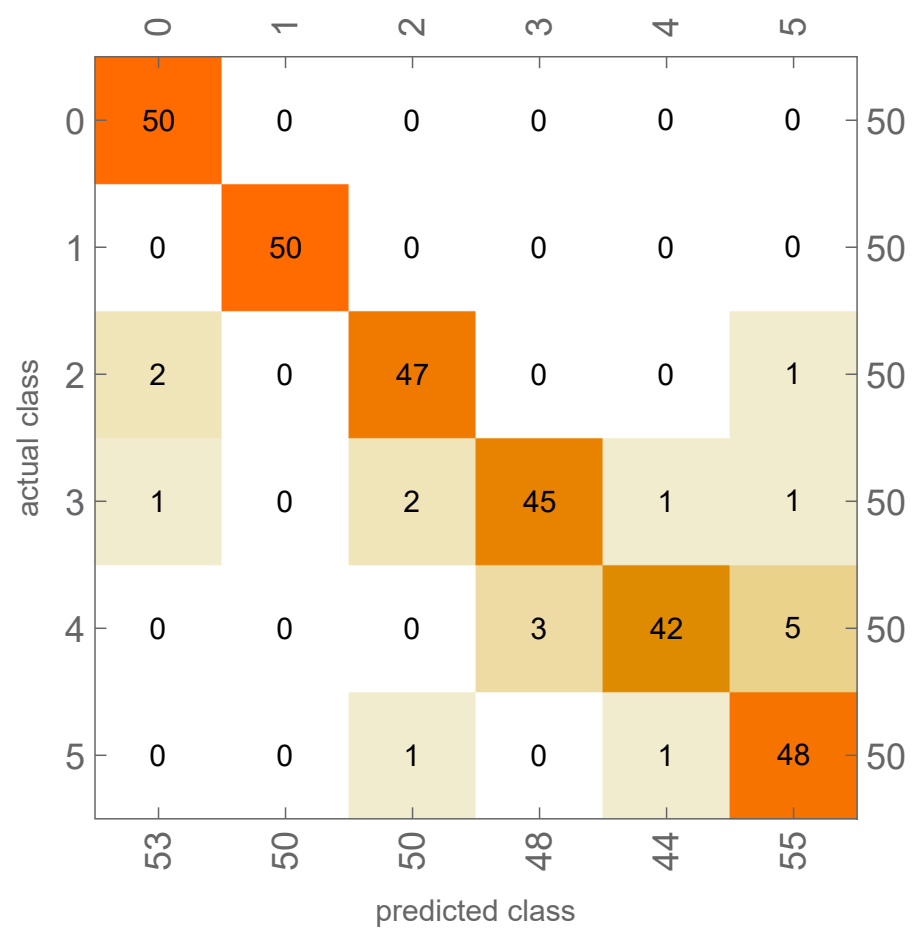

Figure 10: Confusion matrix for a SVM classifier for $-20 \mathrm{~dB}$ SNR (Problem 1). 

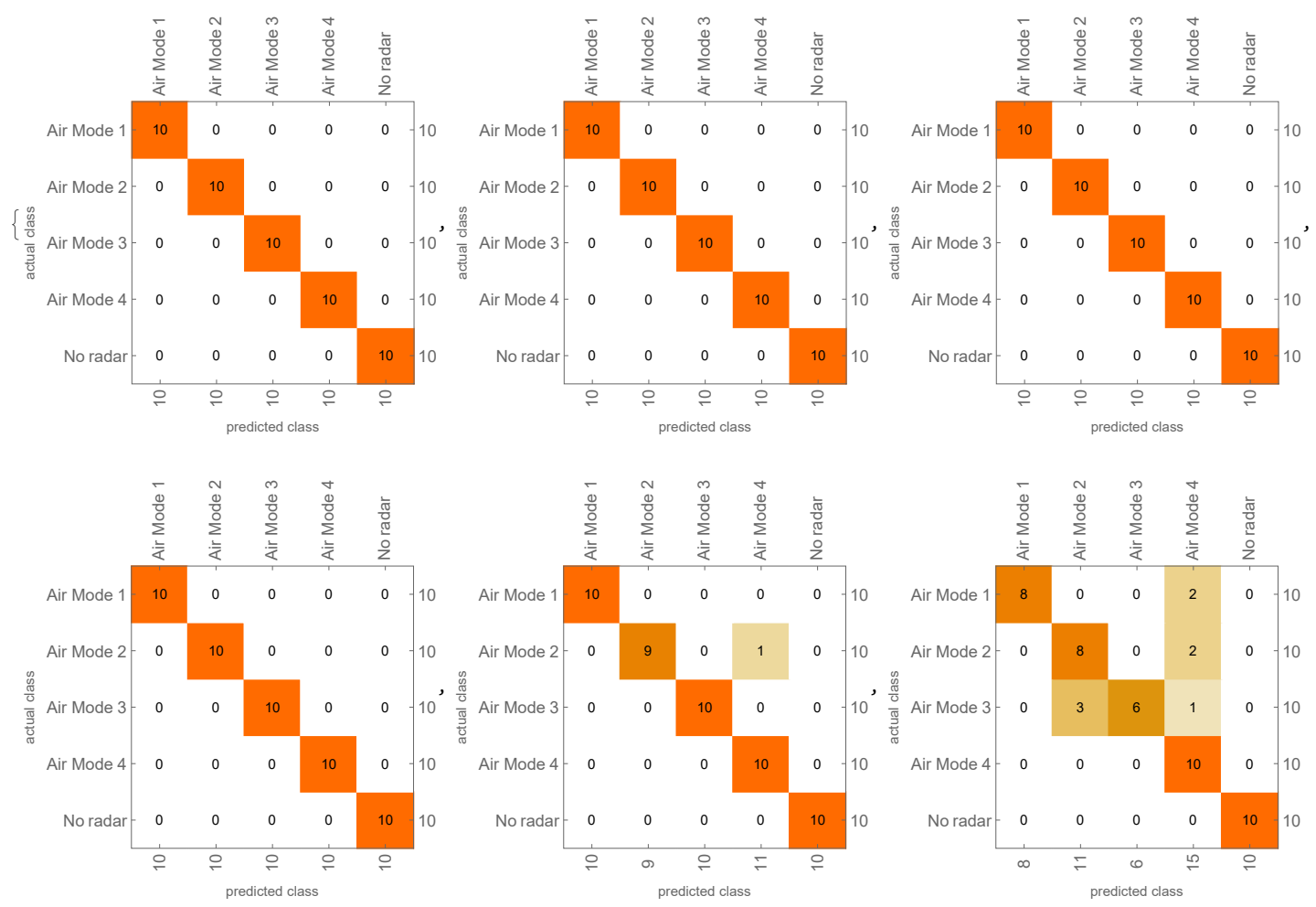

Figure 11: Confusion matrix for a DT classifier of realistic airborne chirp rates at SNRs from $10 \mathrm{~dB}$ (top left), $0 \mathrm{~dB}$ (top mid), $-5 \mathrm{~dB}$ (top right), -10 dB (bottom left), $-15 \mathrm{~dB}$ (bottom mid) and $-20 \mathrm{~dB}$ (bottom right). (Problem 2) 

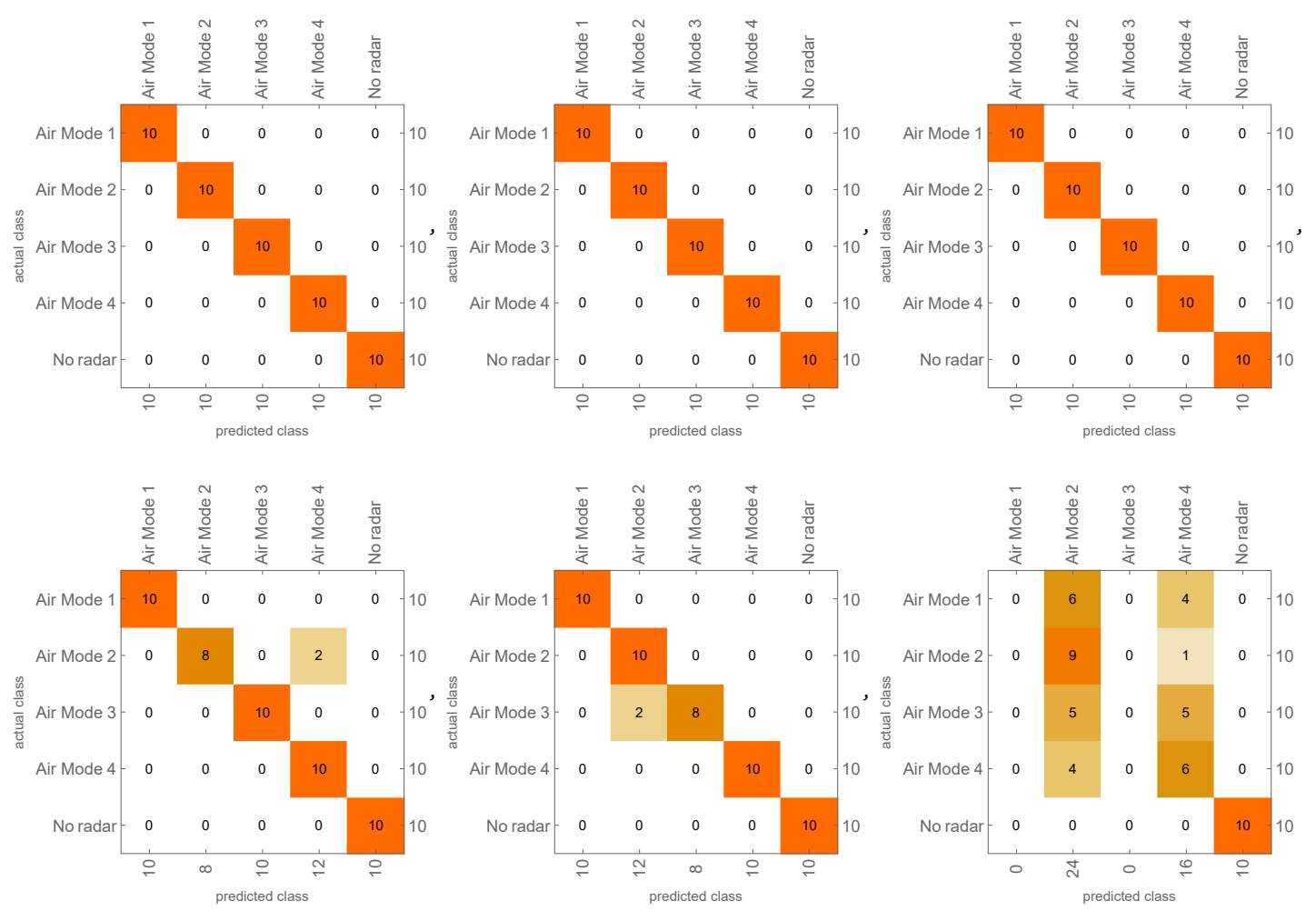

Figure 12: Confusion matrix for a NB classifier of realistic airborne radar chirp rates at SNR $10 \mathrm{~dB}$ (top left), $0 \mathrm{~dB}$ (top mid), $-5 \mathrm{~dB}$ (top right), $-10 \mathrm{~dB}$ (bottom left), $-15 \mathrm{~dB}$ (bottom mid) and $-20 \mathrm{~dB}$ (bottom right). (Problem 2)

realistic chirp rates for airborne radars are shown in Figure 14 for the following SNR values $10 \mathrm{~dB}, 0 \mathrm{~dB},-5 \mathrm{~dB},-10 \mathrm{~dB},-15 \mathrm{~dB}$ and $-20 \mathrm{~dB}$. The confusion matrix shows that the SVM classifier can accurately classify all airborne modes till SNR of -10 dB. Below $-10 \mathrm{~dB}$, the degradation is not as steep as RF. Where as no classifier performs well at $-20 \mathrm{~dB}$.

DT has the best classification rate even at $-20 \mathrm{~dB}$ as shown in Figure. 15. DT has the best performance with respect to $\mathrm{F} 1$ score seen in Figure 16. The performance of SVM is better than NB. The classification performance of NB is worse than DT. The classification performance of $\mathrm{RnF}$ is the worst of all the classifiers considered for this type of radar. This also follows for F1 score. 

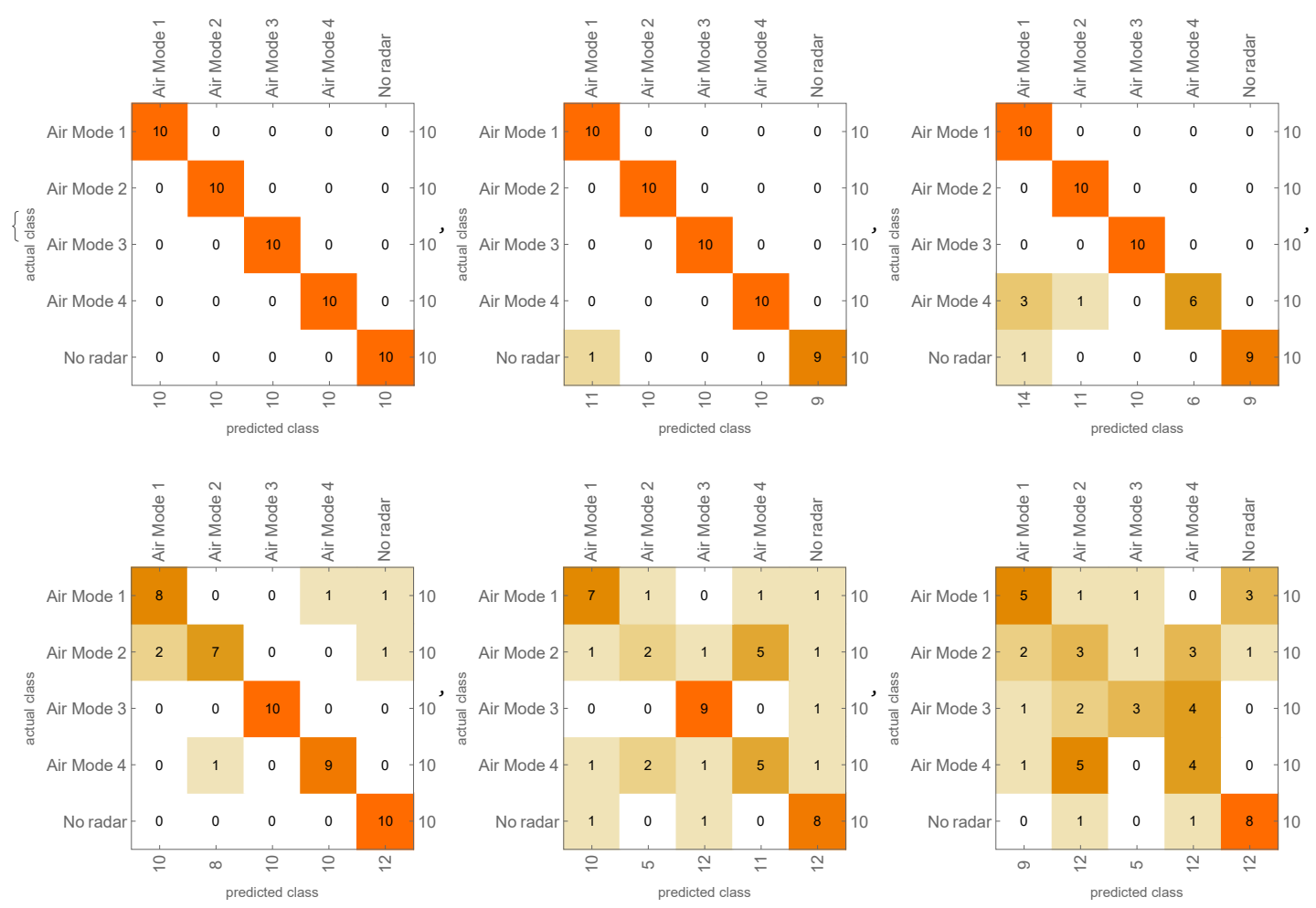

Figure 13: Confusion matrix for a RnF classifier of realistic airborne radar chirp rates at $\mathrm{SNR} 10 \mathrm{~dB}$ (top left), $0 \mathrm{~dB}$ (top mid), $-5 \mathrm{~dB}$ (top right), $-10 \mathrm{~dB}$ (bottom left), $-15 \mathrm{~dB}$ (bottom mid) and $-20 \mathrm{~dB}$ (bottom right). (Problem 2) 

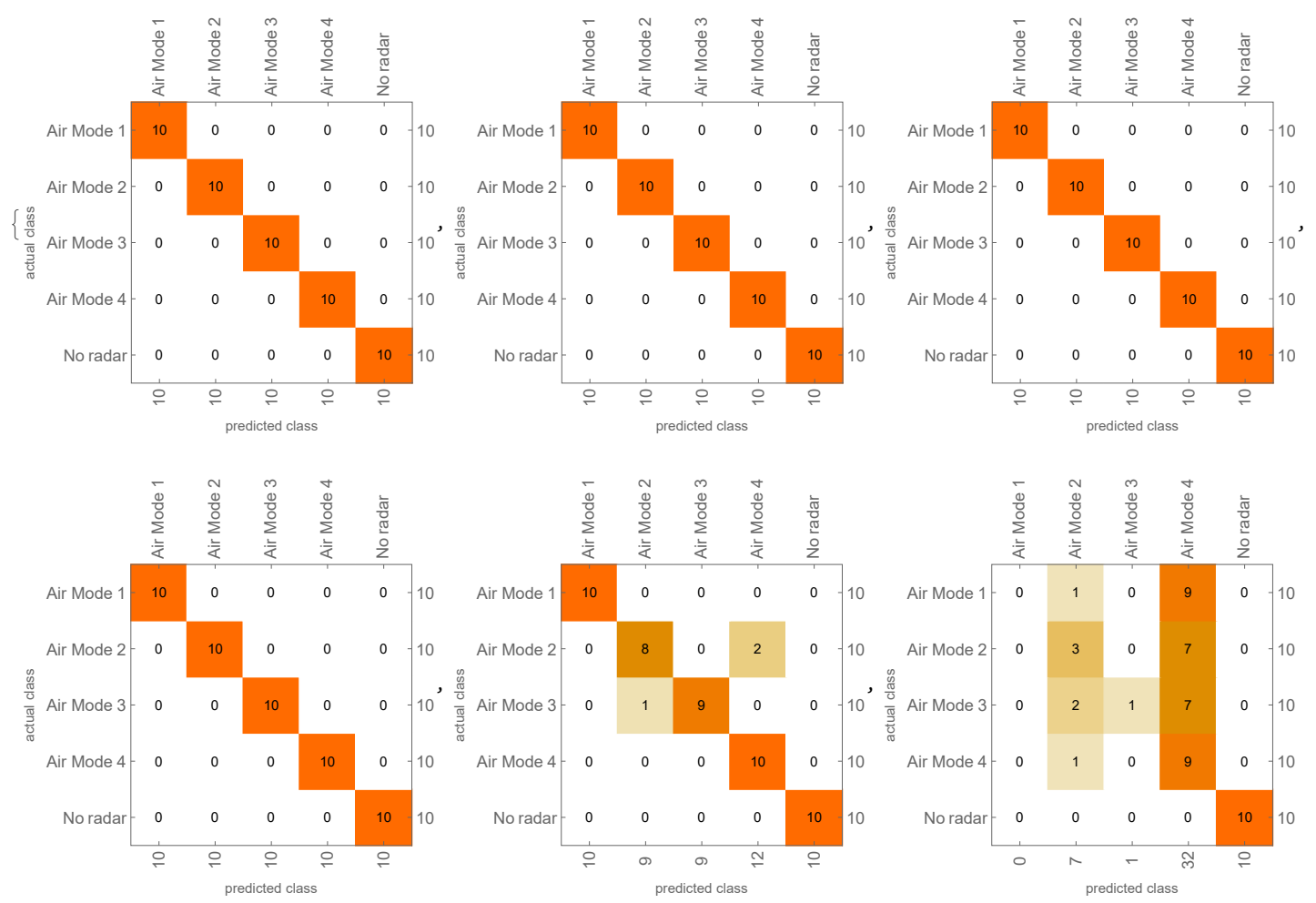

Figure 14: Confusion matrix for a SVM classifier of realistic airborne chirp rates at SNR $10 \mathrm{~dB}$ (top left), $0 \mathrm{~dB}$ (top mid), -5 dB (top right), -10 dB (bottom left), $-15 \mathrm{~dB}$ (bottom mid) and $-20 \mathrm{~dB}$ (bottom right). (Problem 2) 


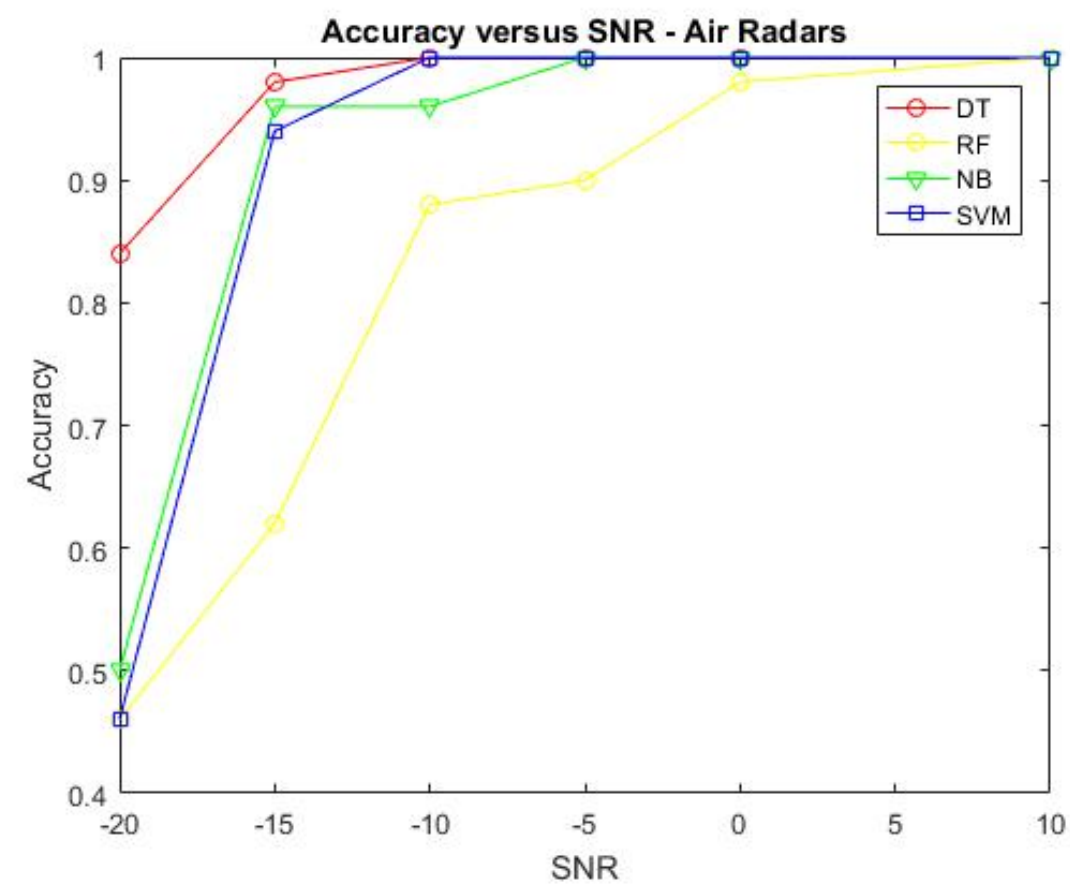

Figure 15: Accuracy versus SNR level - Airborne radar chirp rates. (Problem 2)

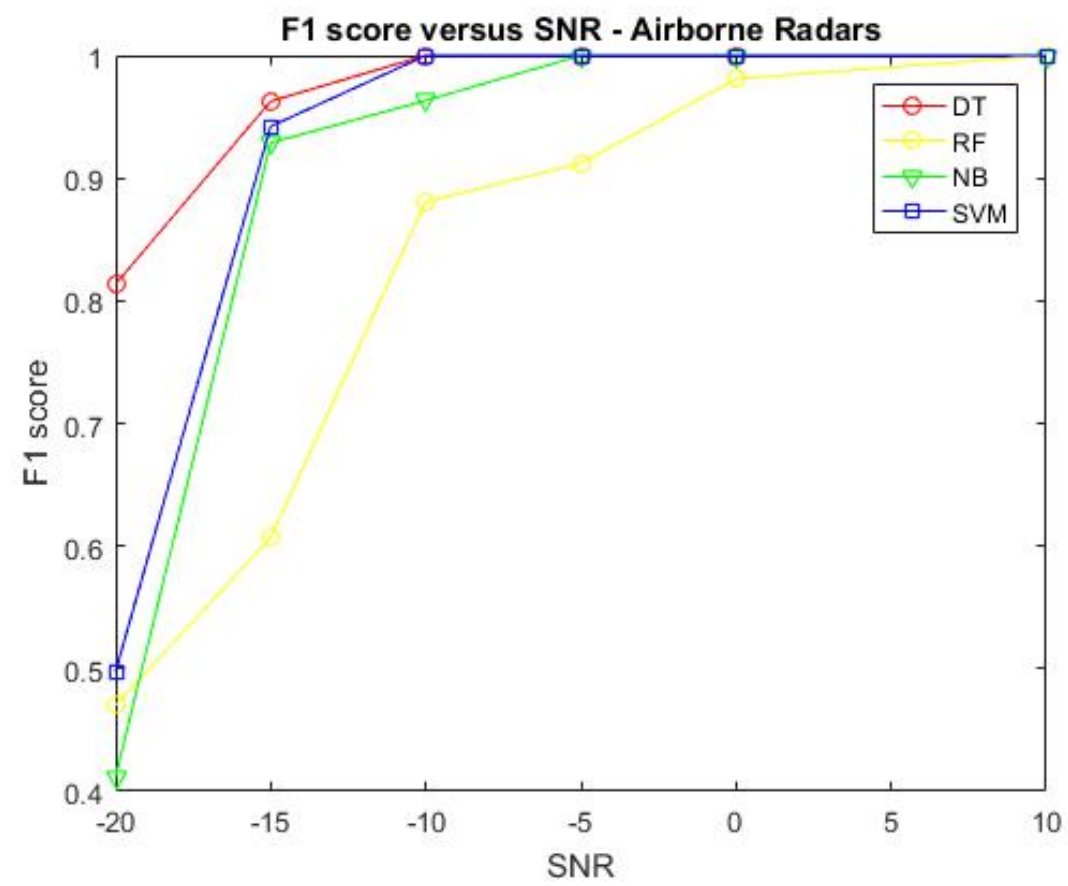

Figure 16: F1 score versus SNR level - Airborne radar chirp rates. (Problem 2) 


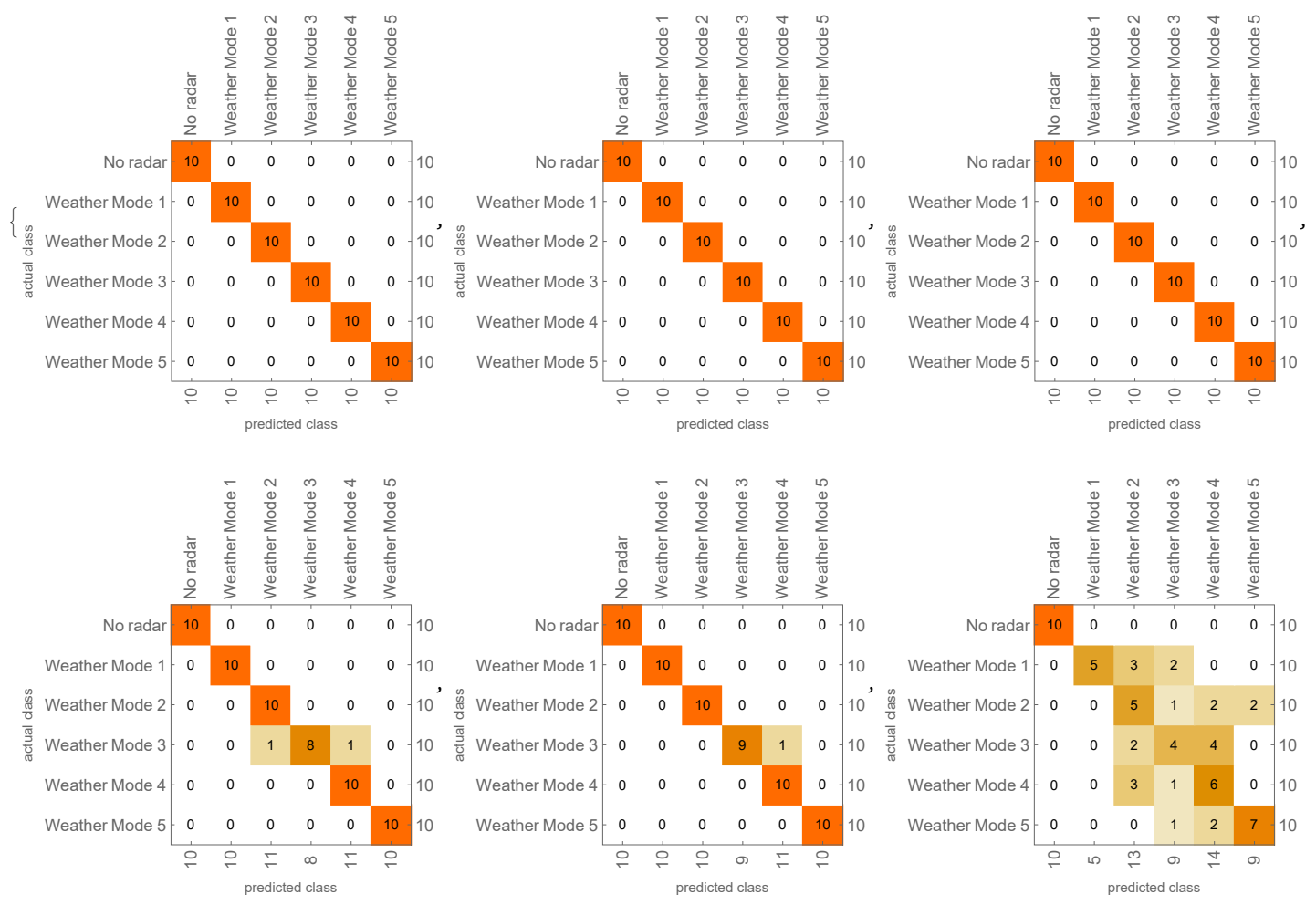

Figure 17: Confusion matrix for a DT classifier of realistic weather radar chirp rates at SNRs $10 \mathrm{~dB}$ (top left), $0 \mathrm{~dB}$ (top mid), $-5 \mathrm{~dB}$ (top right), $-10 \mathrm{~dB}$ (bottom left), $-15 \mathrm{~dB}$ (bottom mid) and $-20 \mathrm{~dB}$ (bottom right). (Problem 3)

\subsubsection{Problem 3: Weather radar realistic chirp results}

The confusion matrix for a DT classifier of realistic weather radar chirp rates in shown in Figure 17 for the following SNR values $10 \mathrm{~dB}, 0 \mathrm{~dB},-5 \mathrm{~dB},-10 \mathrm{~dB},-15 \mathrm{~dB}$ and -20 dB. DT's classification accuracy started dropping from $-5 \mathrm{~dB}$.

The confusion matrix result for a NB classifier of realistic chirp rates for weather radars are shown in Figure 18 for the following SNR values $10 \mathrm{~dB}, 0 \mathrm{~dB},-5 \mathrm{~dB},-10$ $\mathrm{dB},-15 \mathrm{~dB}$ and $-20 \mathrm{~dB}$. NB classification accuracy also started dropping from $-5 \mathrm{~dB}$ SNR.

The confusion matrix result for a $\mathrm{RnF}$ classifier of realistic chirp rates for weather radars are shown in Figure 19 for the following SNR values $10 \mathrm{~dB}, 0 \mathrm{~dB},-5 \mathrm{~dB},-10$ $\mathrm{dB},-15 \mathrm{~dB}$ and $-20 \mathrm{~dB}$. RnF classification accuracy also started dropping from -10 dB SNR. 


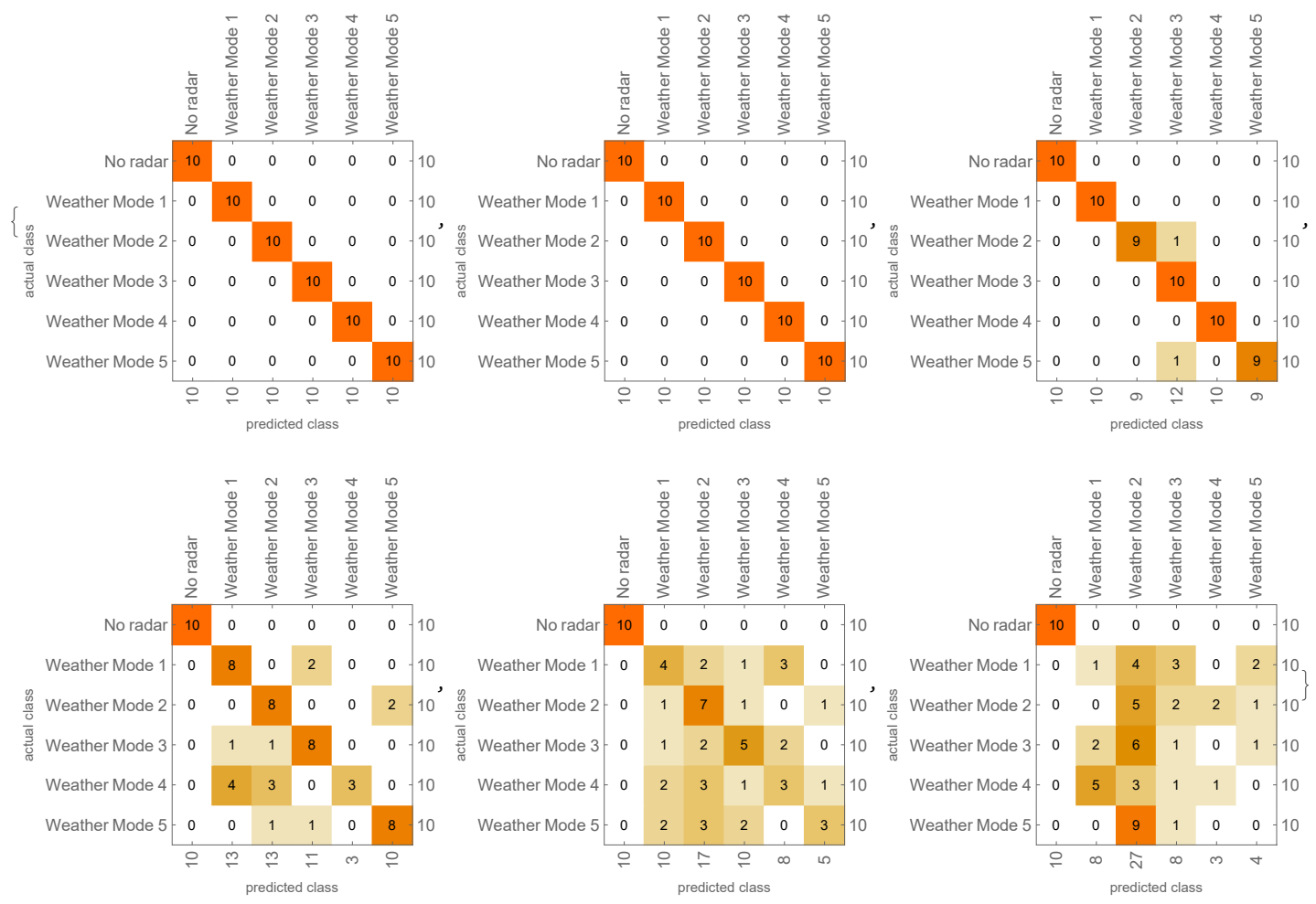

Figure 18: Confusion matrix for a NB classifier of realistic weather radar chirp rates at SNR $10 \mathrm{~dB}$ (top left), $0 \mathrm{~dB}$ (top mid), $-5 \mathrm{~dB}$ (top right), $-10 \mathrm{~dB}$ (bottom left), $-15 \mathrm{~dB}$ (bottom mid) and $-20 \mathrm{~dB}$ (bottom right). (Problem 3) 

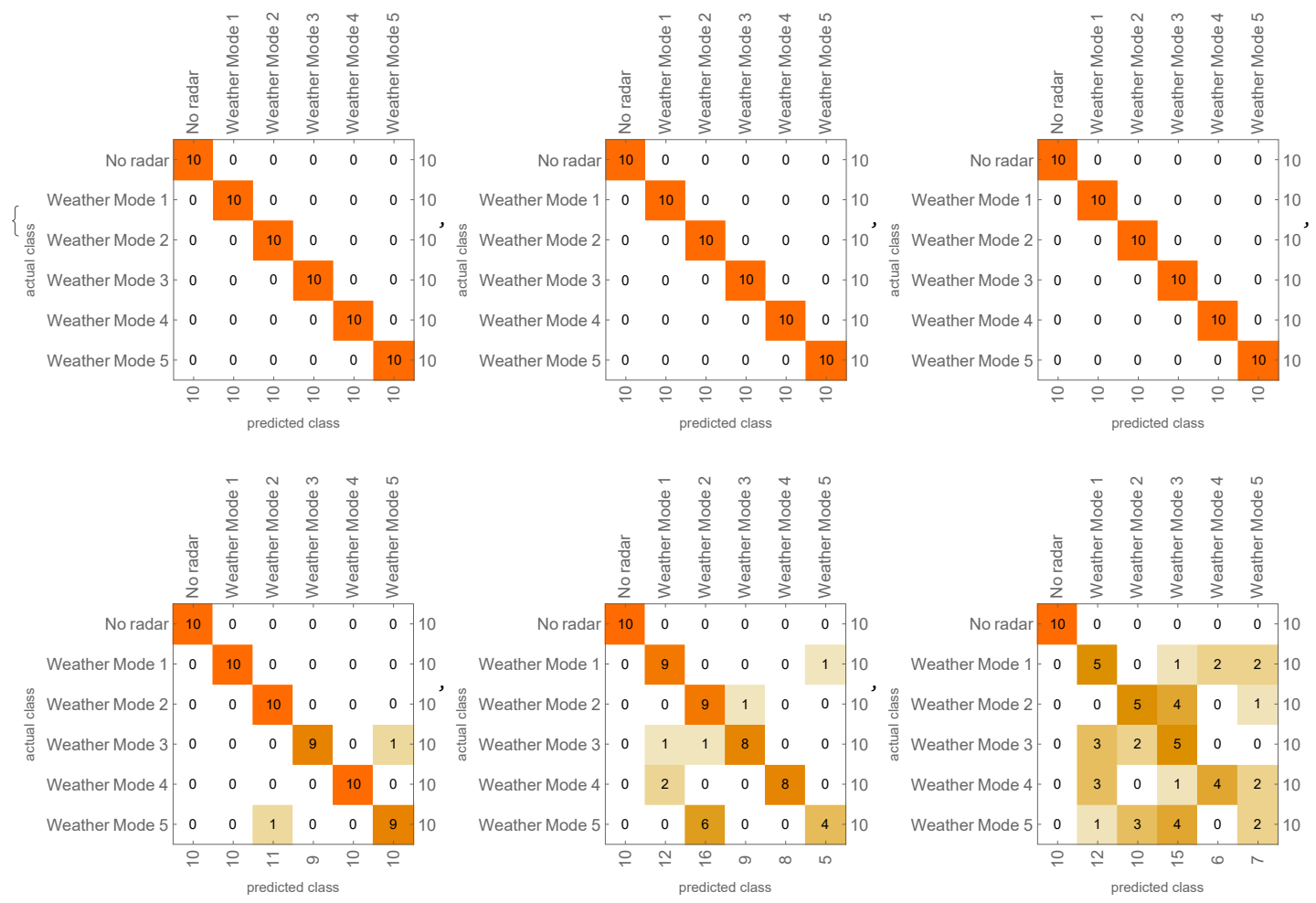

Figure 19: Confusion matrix for a $\mathrm{RnF}$ classifier of realistic weather chirp rates at SNR $10 \mathrm{~dB}$ (top left), $0 \mathrm{~dB}$ (top mid), $-5 \mathrm{~dB}$ (top right), -10 dB (bottom left), $-15 \mathrm{~dB}$ (bottom mid) and $-20 \mathrm{~dB}$ (bottom right). (Problem 3) 

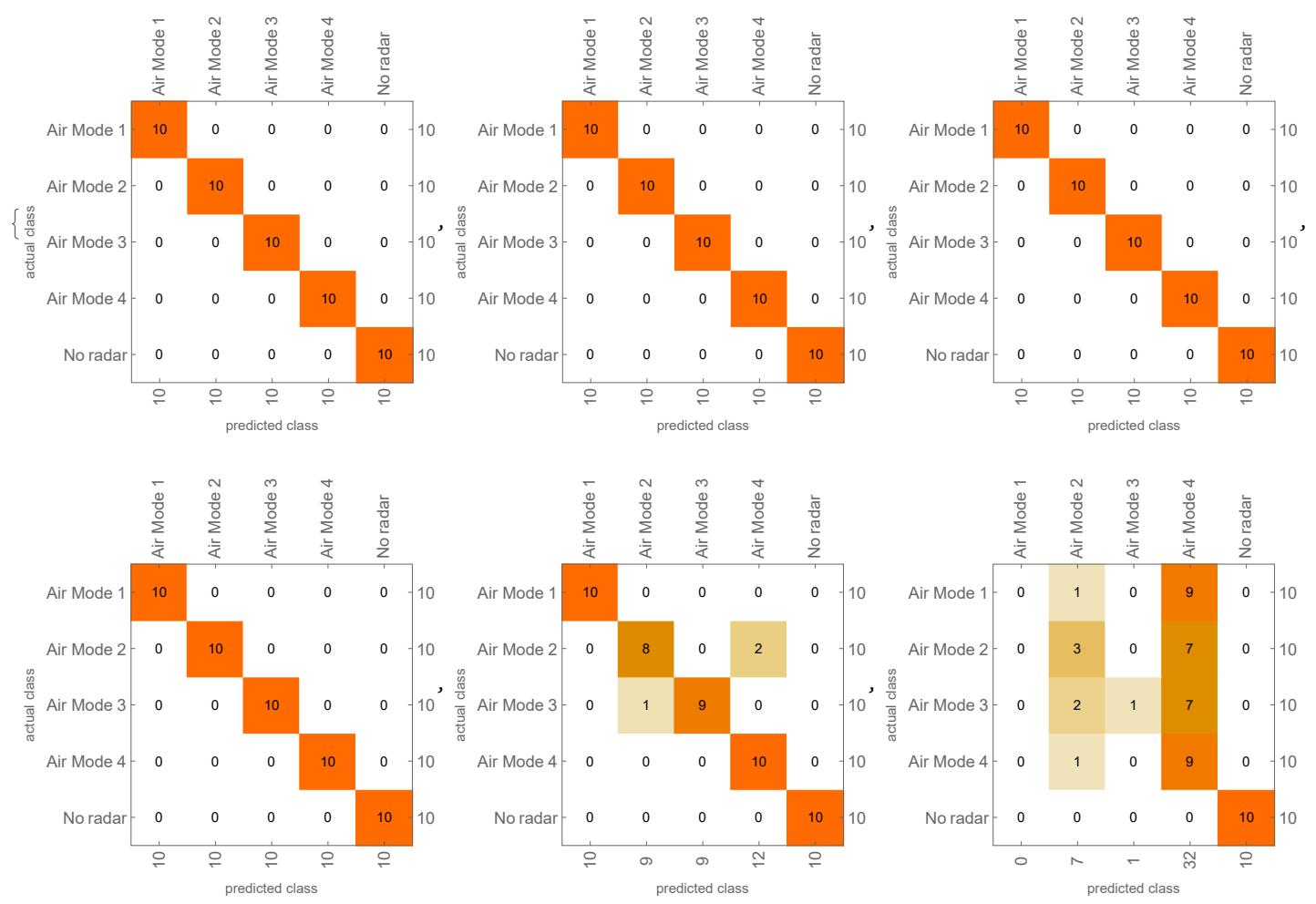

Figure 20: Confusion matrix for a SVM classifier of realistic airborne radar chirp rates at $\mathrm{SNR} 10 \mathrm{~dB}$ (top left), $0 \mathrm{~dB}$ (top mid), $-5 \mathrm{~dB}$ (top right), $-10 \mathrm{~dB}$ (bottom left), $-15 \mathrm{~dB}$ (bottom mid) and $-20 \mathrm{~dB}$ (bottom right). (Problem 3) 
The confusion matrix result for a Support Vector Machine (SVM) classifier of realistic chirp rates for weather radars are shown in Figure 20 for the following SNR values $10 \mathrm{~dB}, 0 \mathrm{~dB},-5 \mathrm{~dB},-10 \mathrm{~dB},-15 \mathrm{~dB}$ and $-20 \mathrm{~dB}$. SVM classification accuracy also started dropping from $-10 \mathrm{~dB}$ SNR.

DT and SVM have the best classification rate for weather radars as shown in Figure.21. RnF is better than NB over all the SNRs. The F1 scores follow the same trend as shown in 22 .

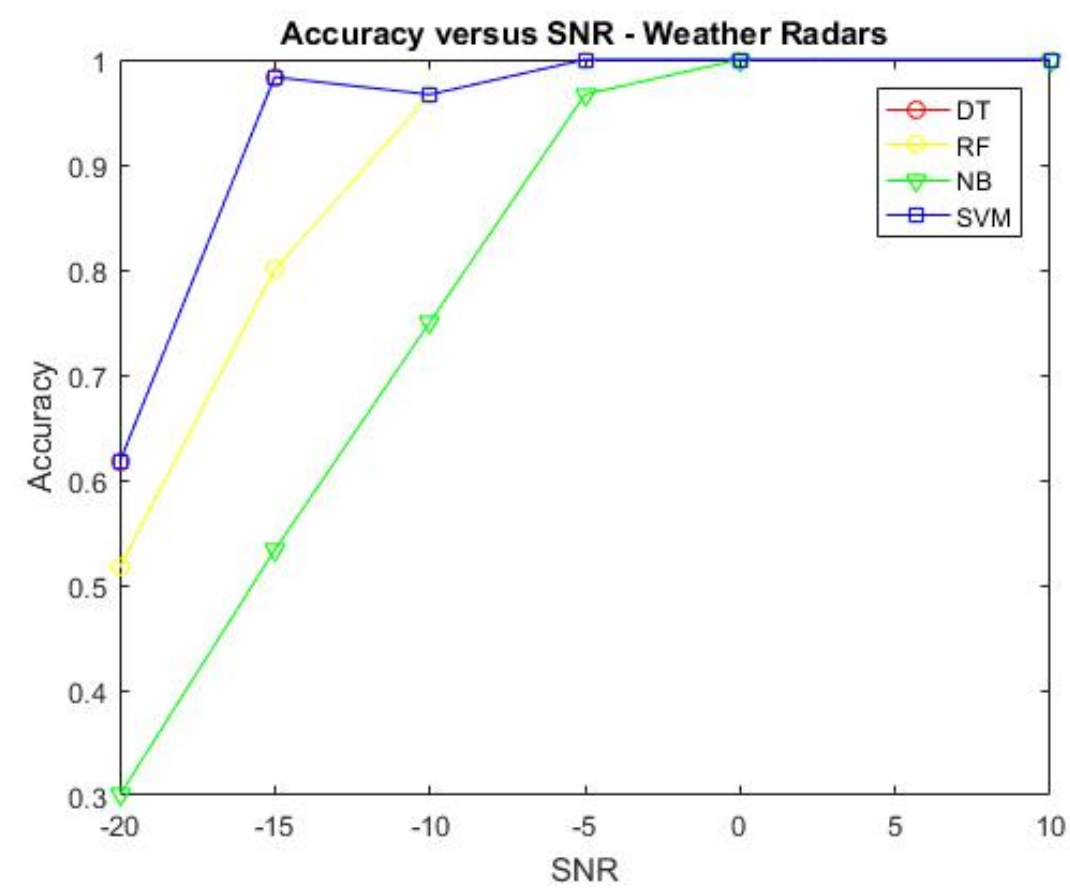

Figure 21: Accuracy versus SNR level - Weather radar chirp rates. (Problem 3)

\subsubsection{Problem 4: Marine radar realistic chirp results}

The confusion matrix for a DT classifier of realistic marine radar chirp rates in shown in Figure 23 for the following SNR values $10 \mathrm{~dB}, 0 \mathrm{~dB},-5 \mathrm{~dB},-10 \mathrm{~dB},-15 \mathrm{~dB}$ and -20 dB. DT's classification accuracy started dropping from $-15 \mathrm{~dB}$.

The confusion matrix result for a NB classifier of realistic chirp rates for marine radars are shown in Figure 24 for the following SNR values $10 \mathrm{~dB}, 0 \mathrm{~dB},-5 \mathrm{~dB},-10$ $\mathrm{dB},-15 \mathrm{~dB}$ and $-20 \mathrm{~dB}$. NB classification accuracy also started dropping from $-5 \mathrm{~dB}$ SNR. 


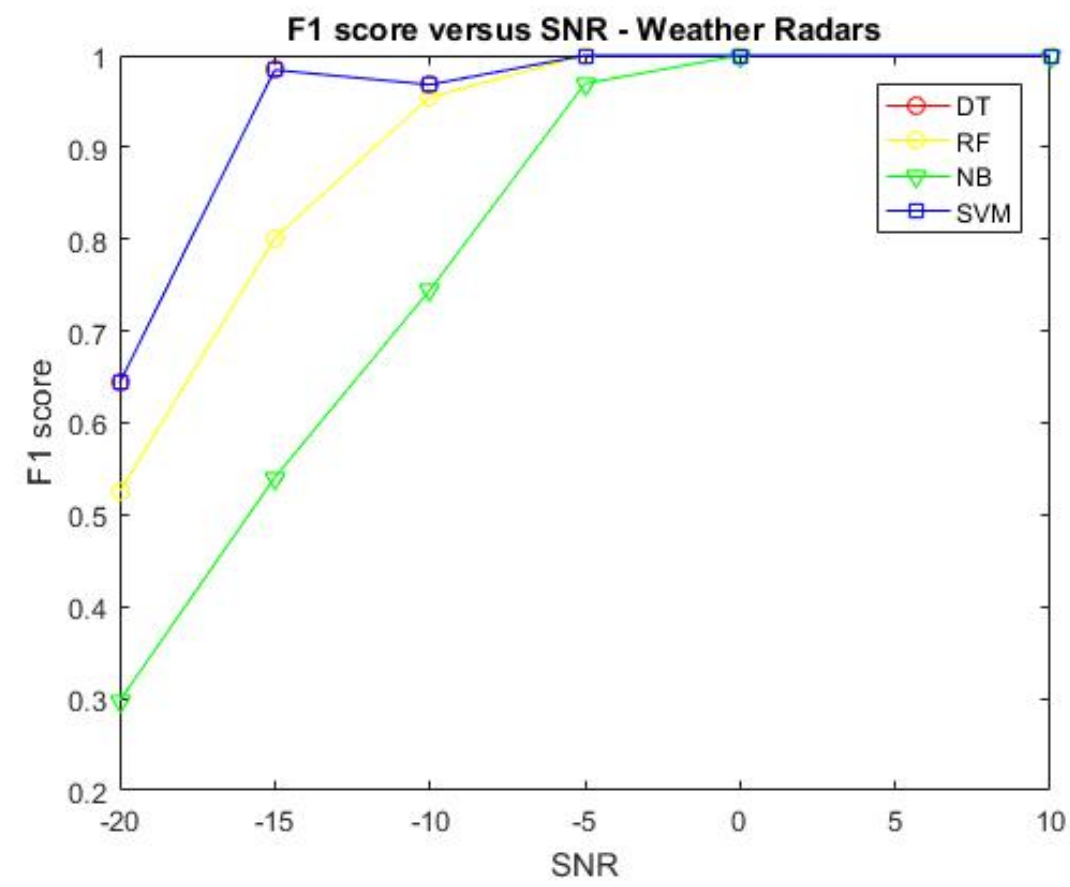

Figure 22: F1 score versus SNR level - Weather radar chirp rates. (Problem 3)

The confusion matrix result for a $\mathrm{RnF}$ classifier of realistic chirp rates for marine radars are shown in Figure 25 for the following SNR values $10 \mathrm{~dB}, 0 \mathrm{~dB},-5 \mathrm{~dB},-10$ $\mathrm{dB},-15 \mathrm{~dB}$ and $-20 \mathrm{~dB}$. RnF classification accuracy also started dropping from -10 dB SNR.

The confusion matrix result for a Support Vector Machine (SVM) classifier of realistic chirp rates for marine radars are shown in Figure 26 for the following SNR values $10 \mathrm{~dB}, 0 \mathrm{~dB},-5 \mathrm{~dB},-10 \mathrm{~dB},-15 \mathrm{~dB}$ and $-20 \mathrm{~dB}$. SVM classification accuracy started dropping from -15 dB SNR. Note that "Radar 10" to "Radar 15" in Figure 26 is the same as "Marine Mode 1" to "Marine Mode 5".

DT has the best classification rate for marine radars as shown in Figure 27. RnF is better than NB over all the SNRs. The F1 scores follow the same trend as shown in 28. 

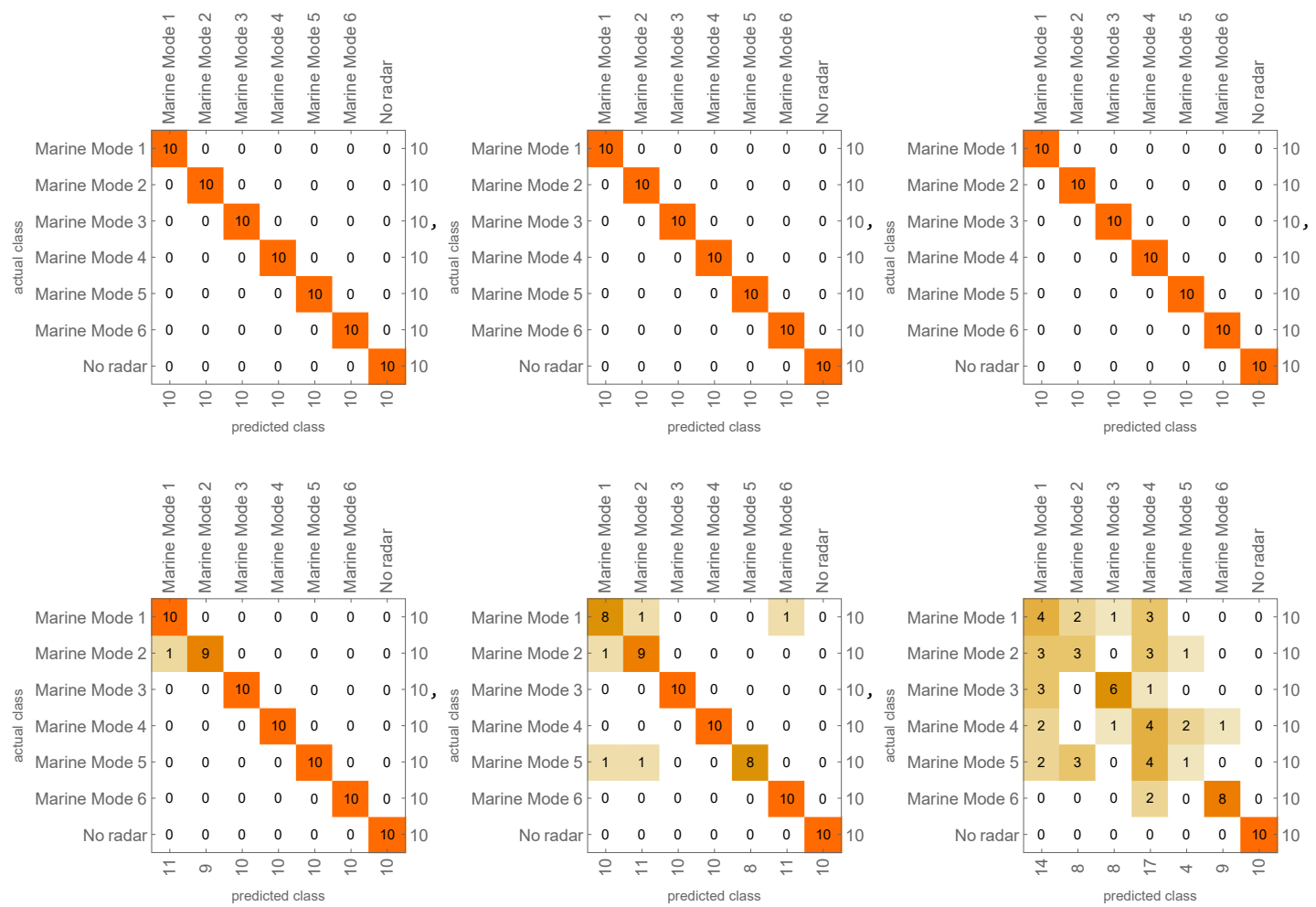

Figure 23: Confusion matrix for a DT classifier of realistic marine radar chirp rates at SNR $10 \mathrm{~dB}$ (top left), $0 \mathrm{~dB}$ (top mid), $-5 \mathrm{~dB}$ (top right), $-10 \mathrm{~dB}$ (bottom left), $-15 \mathrm{~dB}$ (bottom mid) and $-20 \mathrm{~dB}$ (bottom right). (Problem 4) 

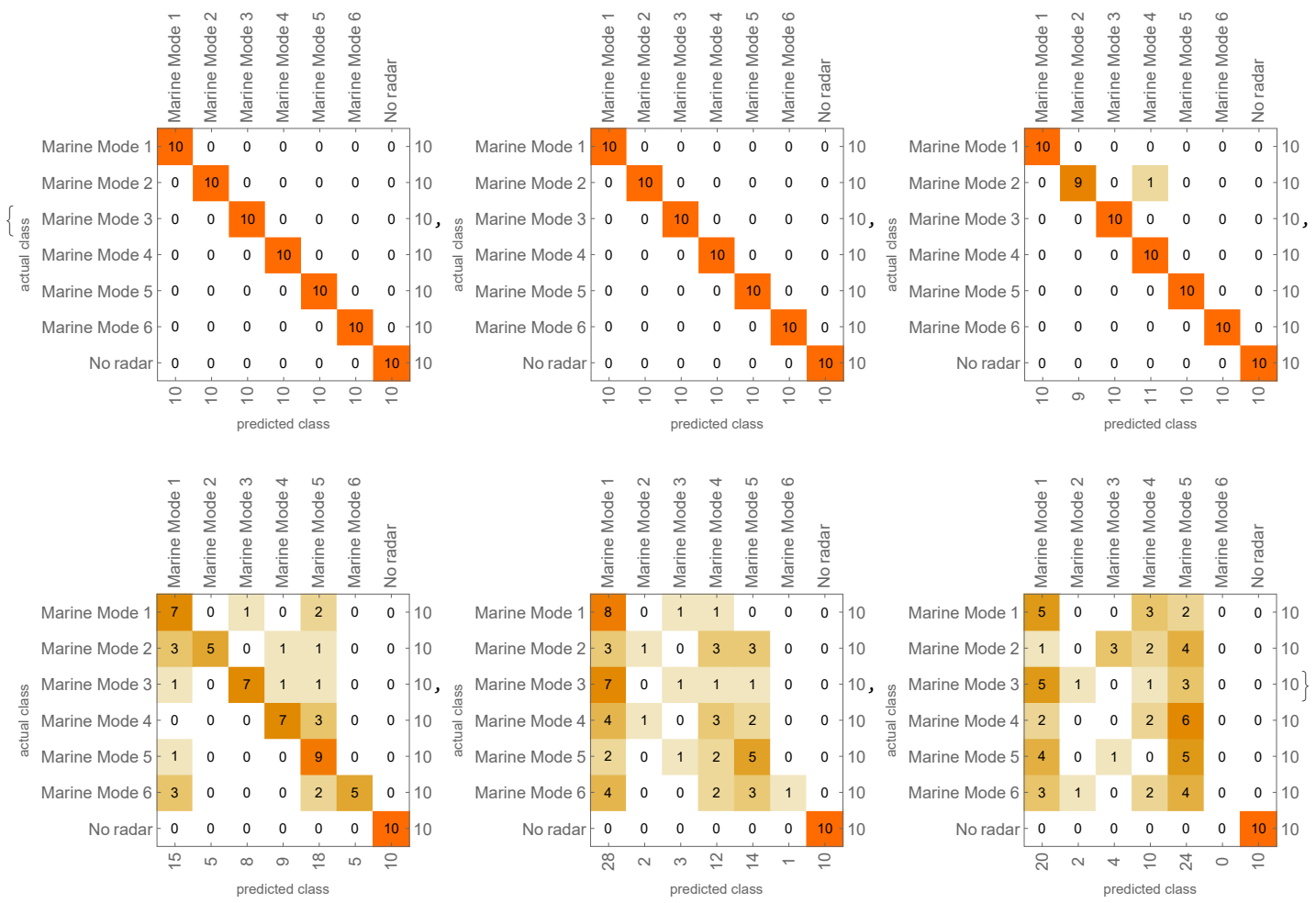

Figure 24: Confusion matrix for a NB classifier of realistic marine radar chirp rates at SNR $10 \mathrm{~dB}$ (top left), $0 \mathrm{~dB}$ (top mid), $-5 \mathrm{~dB}$ (top right), $-10 \mathrm{~dB}$ (bottom left), $-15 \mathrm{~dB}$ (bottom mid) and $-20 \mathrm{~dB}$ (bottom right). (Problem 4) 

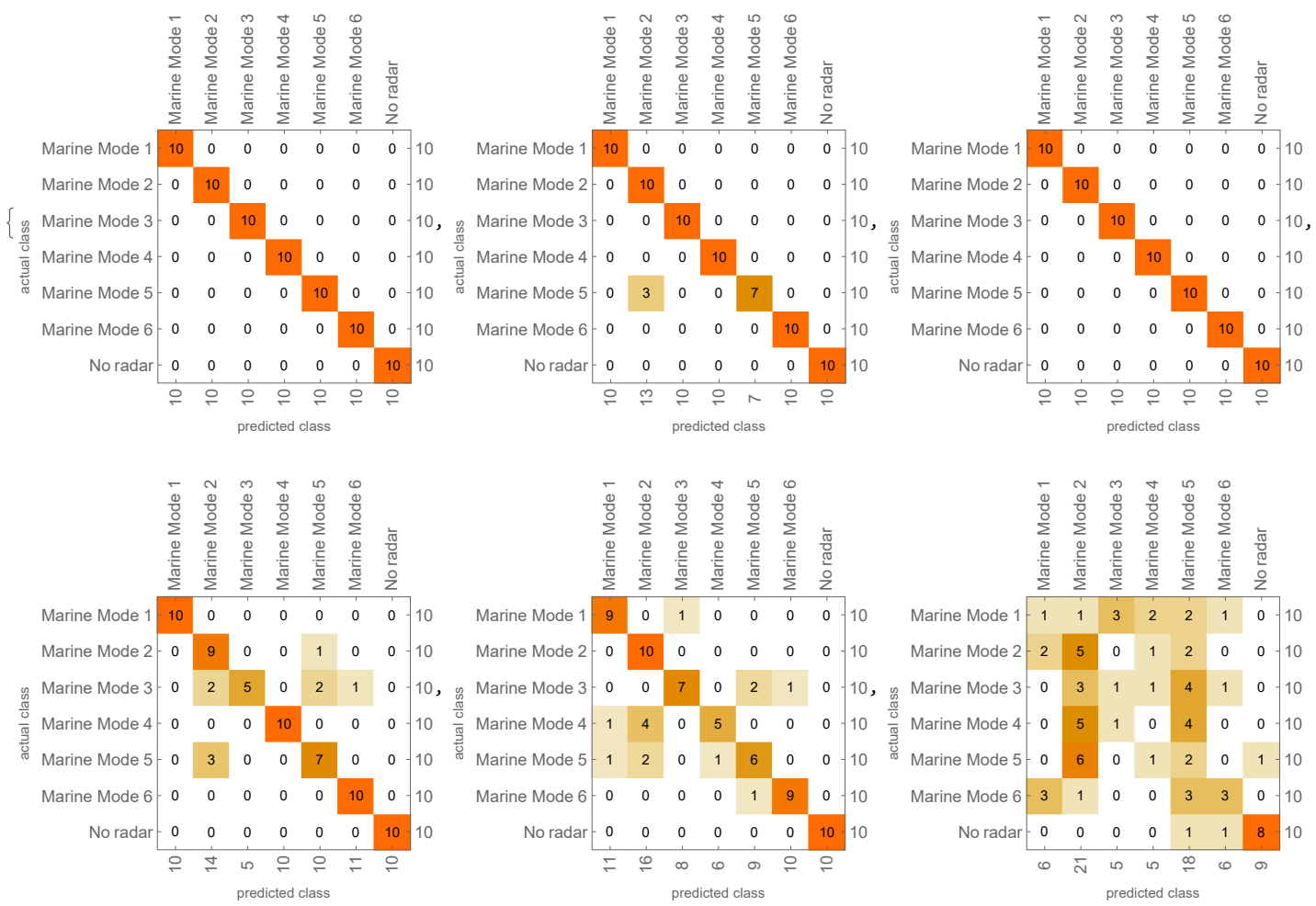

Figure 25: Confusion matrix for a $\mathrm{RnF}$ classifier of realistic marine radar chirp rates at SNR $10 \mathrm{~dB}$ (top left), $0 \mathrm{~dB}$ (top mid), $-5 \mathrm{~dB}$ (top right), $-10 \mathrm{~dB}$ (bottom left), $-15 \mathrm{~dB}$ (bottom mid) and $-20 \mathrm{~dB}$ (bottom right). (Problem 4) 

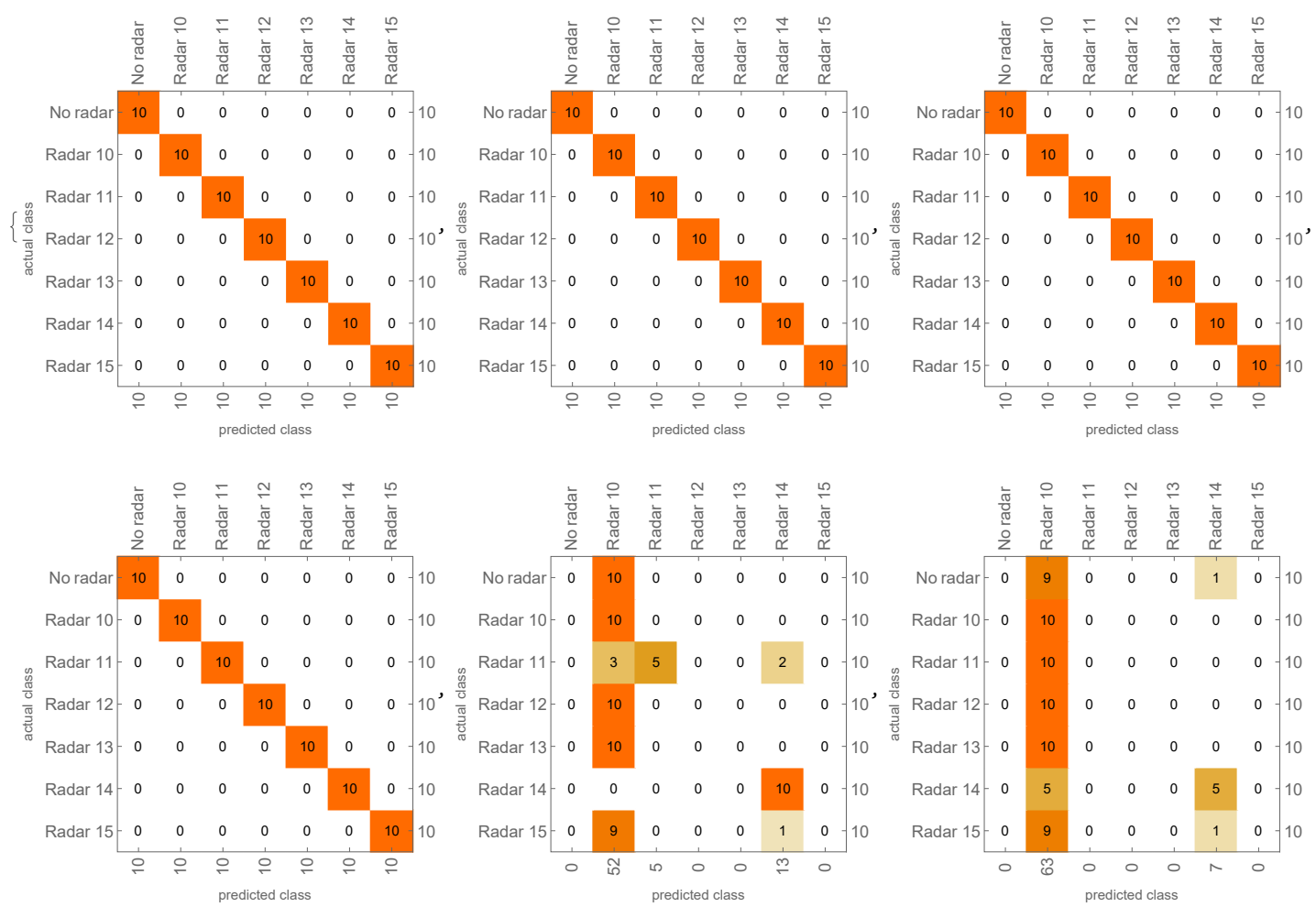

Figure 26: Confusion matrix for a SVM classifier of realistic marine radar chirp rates at SNR $10 \mathrm{~dB}$ (top left), $0 \mathrm{~dB}$ (top mid) , $-5 \mathrm{~dB}$ (top right), $-10 \mathrm{~dB}$ (bottom left), $-15 \mathrm{~dB}$ (bottom mid) and $-20 \mathrm{~dB}$ (bottom right). (Problem 4) 


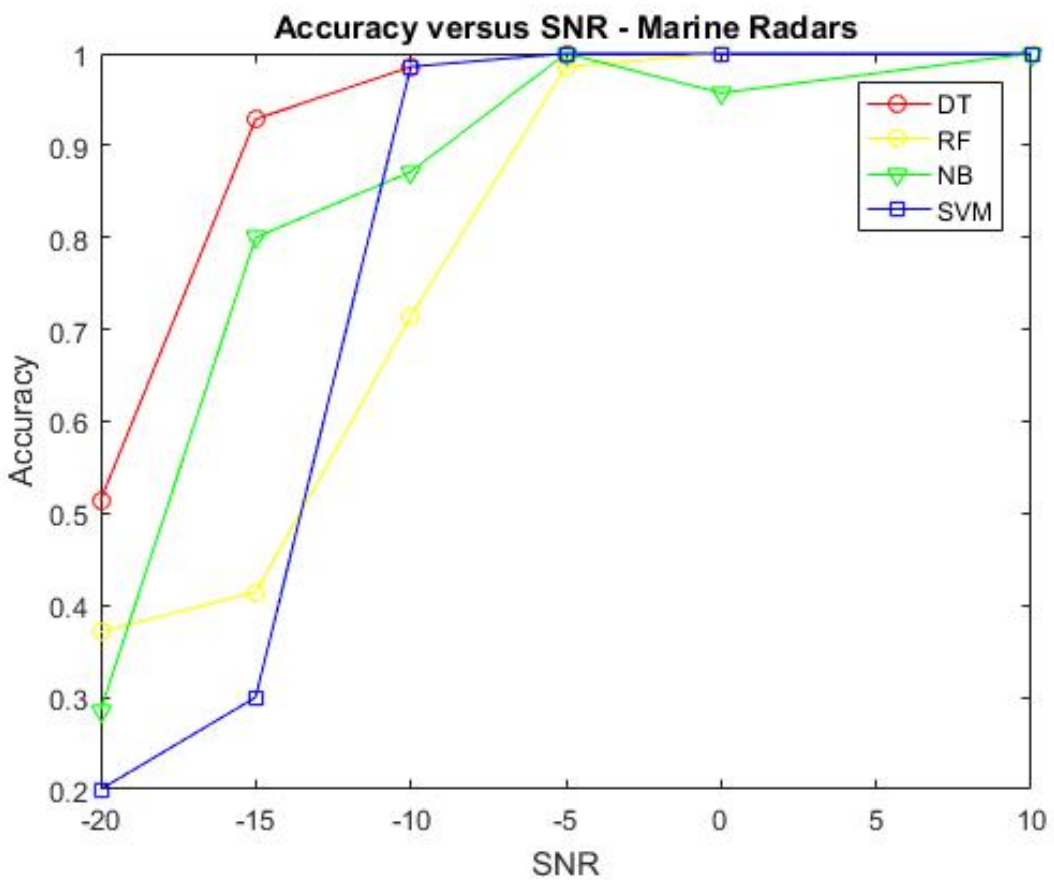

Figure 27: Accuracy versus SNR level - Marine radar chirp rates. (Problem 4)

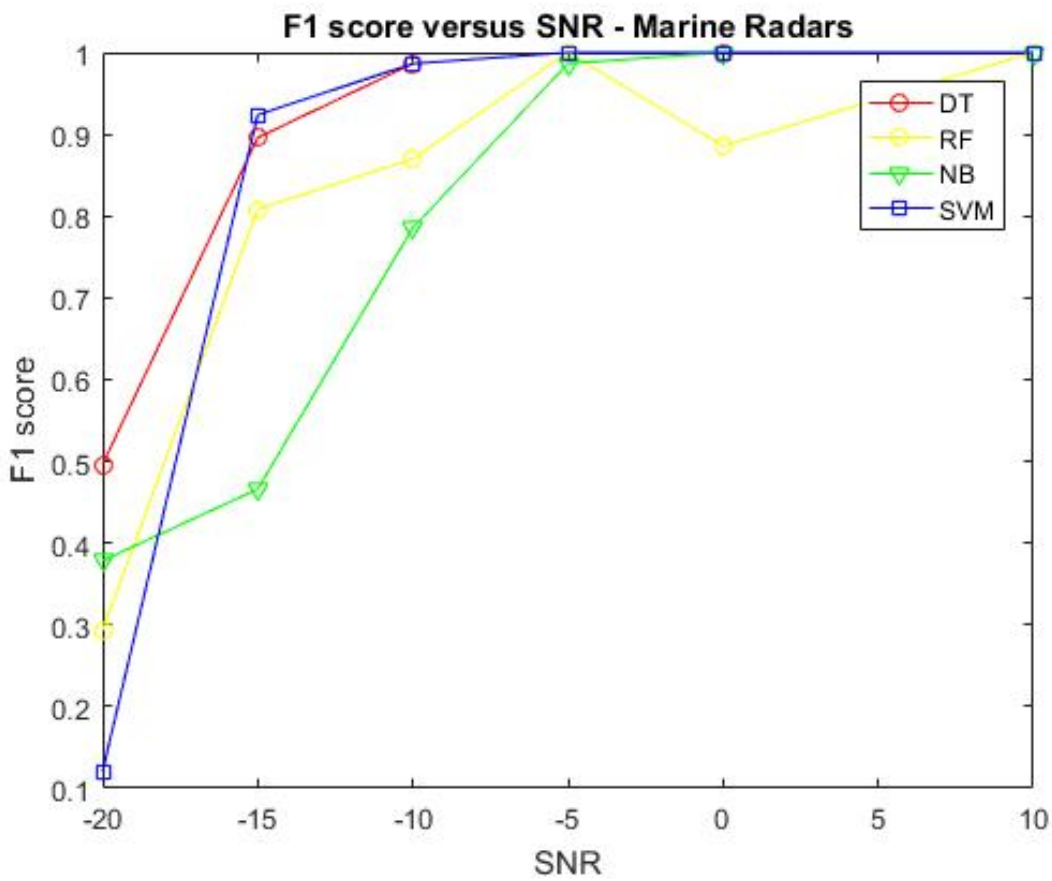

Figure 28: F1 score versus SNR level - Marine radar chirp rates. (Problem 4) 


\subsubsection{Problem 5: All realistic radar chirp rates results}

The confusion matrix for a DT classifier of realistic chirp rates for all radars in shown in Figure 29 for the following SNR values $10 \mathrm{~dB}, 0 \mathrm{~dB},-5 \mathrm{~dB},-10 \mathrm{~dB},-15 \mathrm{~dB}$ and -20 dB. DT's classification accuracy started dropping from $-10 \mathrm{~dB}$.

The confusion matrix result for a NB classifier of realistic chirp rates for all radars are shown in Figure 30 for the following SNR values $10 \mathrm{~dB}, 0 \mathrm{~dB},-5 \mathrm{~dB},-10 \mathrm{~dB},-15$ $\mathrm{dB}$ and $-20 \mathrm{~dB}$. NB classification accuracy also started dropping from -10 dB SNR.

The confusion matrix result for a SVM classifier realistic chirp rates for all radars are shown in Figure 31 for the following SNR values $10 \mathrm{~dB}, 0 \mathrm{~dB},-5 \mathrm{~dB},-10 \mathrm{~dB},-15$ $\mathrm{dB}$ and $-20 \mathrm{~dB}$. SVM classification accuracy also started dropping from -10 dB SNR.

DT, NB, and SVM have almost identical performance in terms of classification rate for all radars till SNR of $-5 \mathrm{~dB}$ as shown in Figure 32. The deterioration of the DT is less than NB and SVM. The F1 scores follow the same trend as shown in Figure 33. The performance of three ML classifiers was analysed for all realistic radar chirp rates. The RnF ML classifier was not evaluated as its performance was significantly worse than the other three classifiers at higher SNR values.

The marine radars have the same sampling frequency and center frequency with only changes in the pulsewidth. Secondly, there are three pairs of pulse widths where the only difference in each pairing is a change in the bandwidth. These pairs are marine mode 1 and marine mode 4, marine mode 2 and marine mode 5 and marine mode 3 , and marine mode 6 . Since there are only differences between these pairs, the classification results are poorer generally and more so in the more of noise. It is clear that the marine radar chirp rates are the most difficult type to classify by all the algorithms. Future work will investigate if more advanced machine learning algorithms with feature selection can improve upon the results in this thesis.

\subsubsection{Problem 6: Robustness of the classifiers against win- dowing imperfections}

As noted previously, the intercept receiver may not be operating when the transmitted pulse is received at the receiver. When the receiver is turned on, it might miss a 

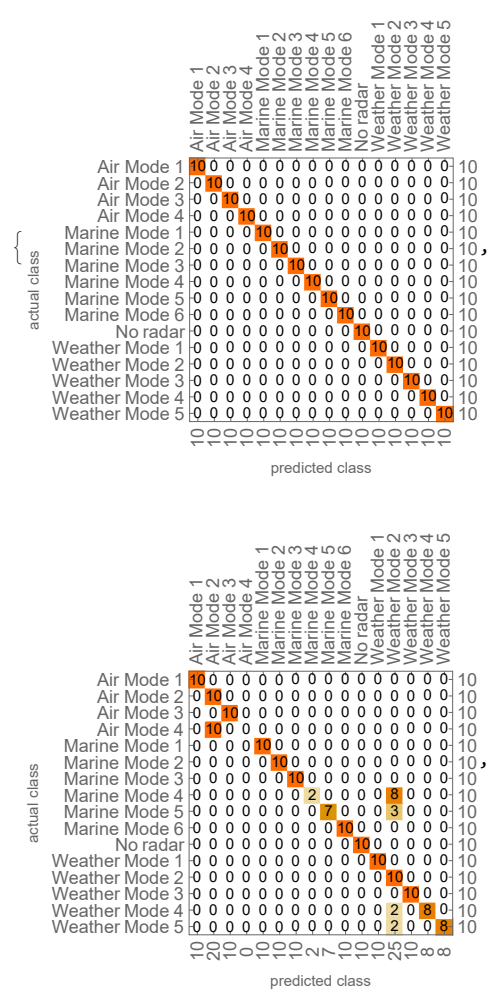
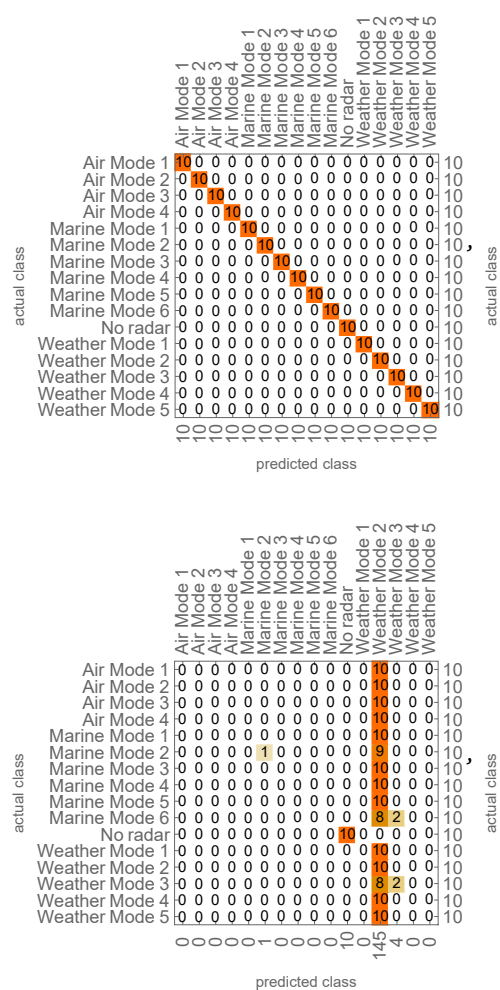
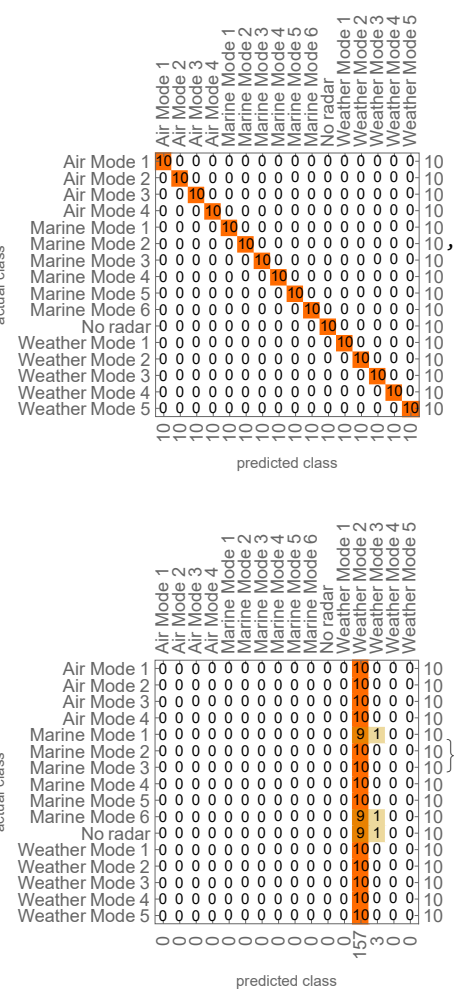

Figure 29: Confusion matrix for a DT classifier of all realistic radar chirp rates at SNR $10 \mathrm{~dB}$ (top left), $0 \mathrm{~dB}$ (top mid), $-5 \mathrm{~dB}$ (top right), -10 dB (bottom left), $-15 \mathrm{~dB}$ (bottom mid) and $-20 \mathrm{~dB}$ (bottom right). (Problem 5) 

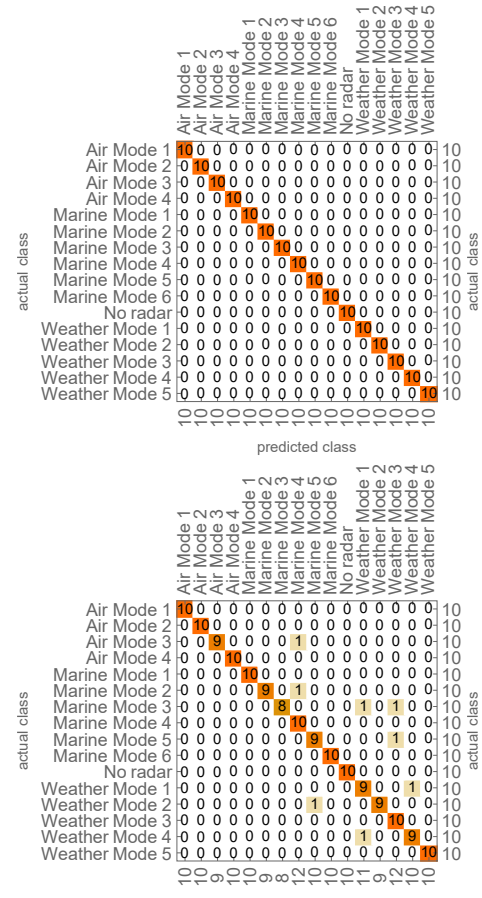

predicted class

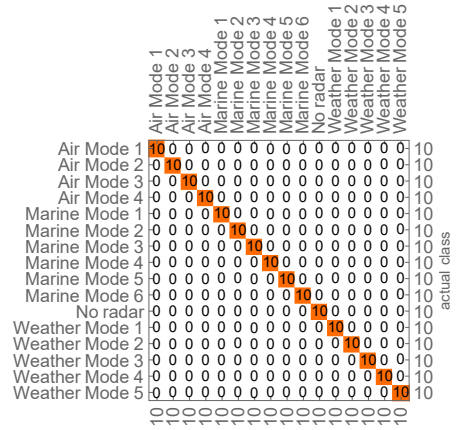

으유으으으으으으유으으으으으으우

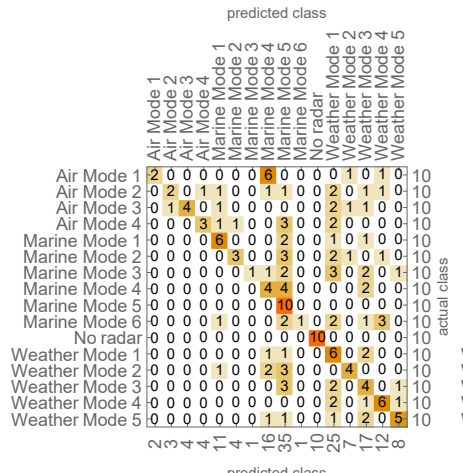

predicted class
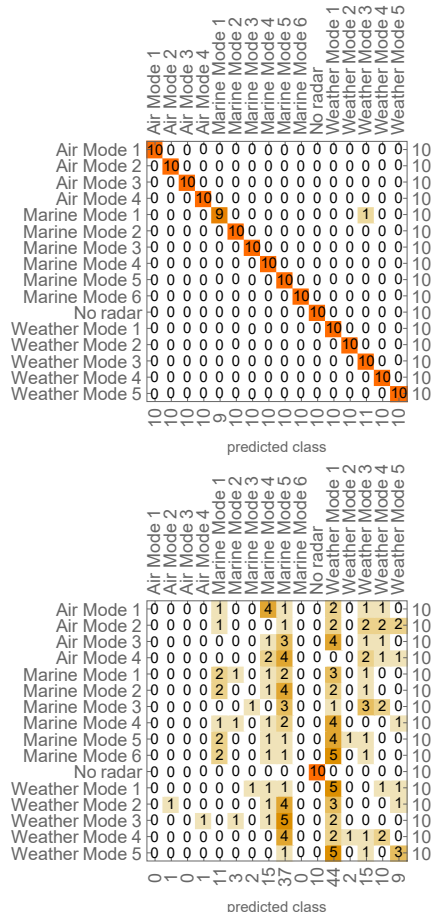

predicted class

Figure 30: Confusion matrix for a NB classifier of all realistic radar chirp rates at SNR $10 \mathrm{~dB}$ (top left), $0 \mathrm{~dB}$ (top mid), $-5 \mathrm{~dB}$ (top right), -10 dB (bottom left), $-15 \mathrm{~dB}$ (bottom mid) and $-20 \mathrm{~dB}$ (bottom right). (Problem 5)
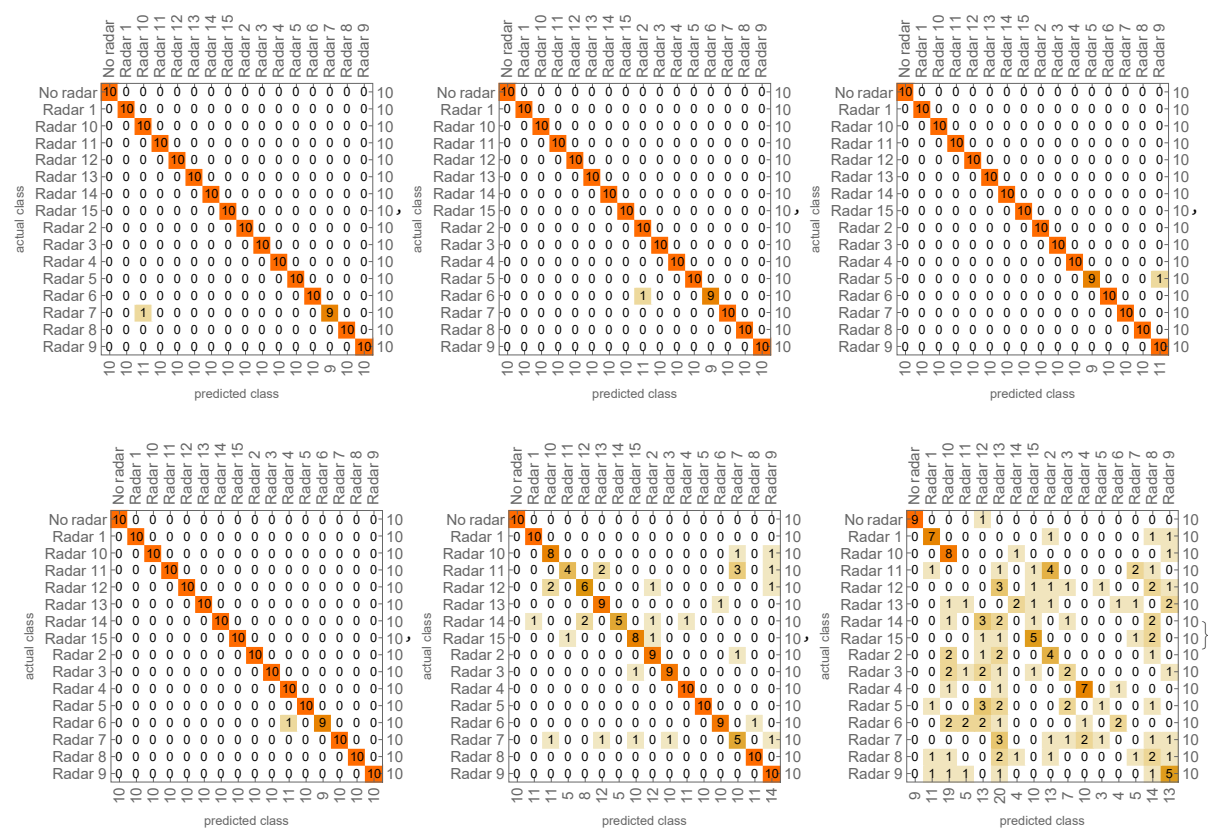

Figure 31: Confusion matrix for a SVM classifier of all realistic radar chirp rates at SNR $10 \mathrm{~dB}$ (top left), $0 \mathrm{~dB}$ (top mid), $-5 \mathrm{~dB}$ (top right), -10 dB (bottom left), $-15 \mathrm{~dB}$ (bottom mid) and $-20 \mathrm{~dB}$ (bottom right). (Problem 5) 


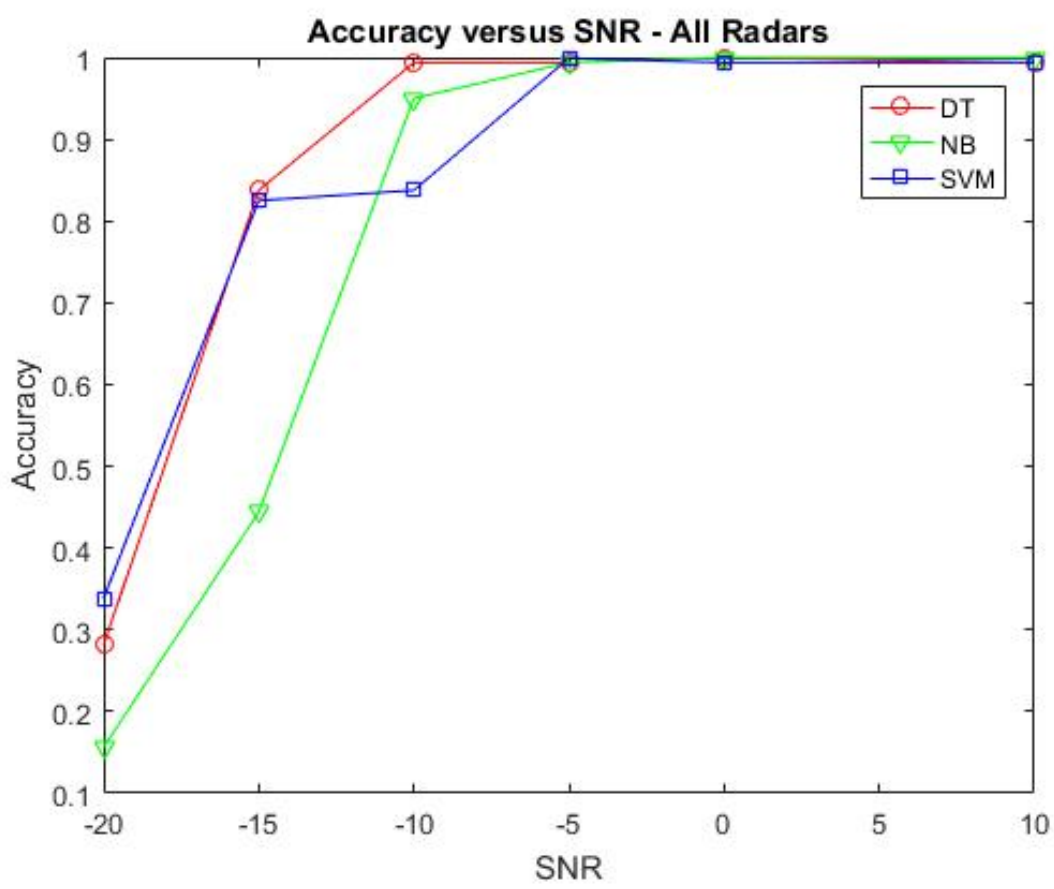

Figure 32: Accuracy versus SNR level - All radar chirp rates. (Problem 5)

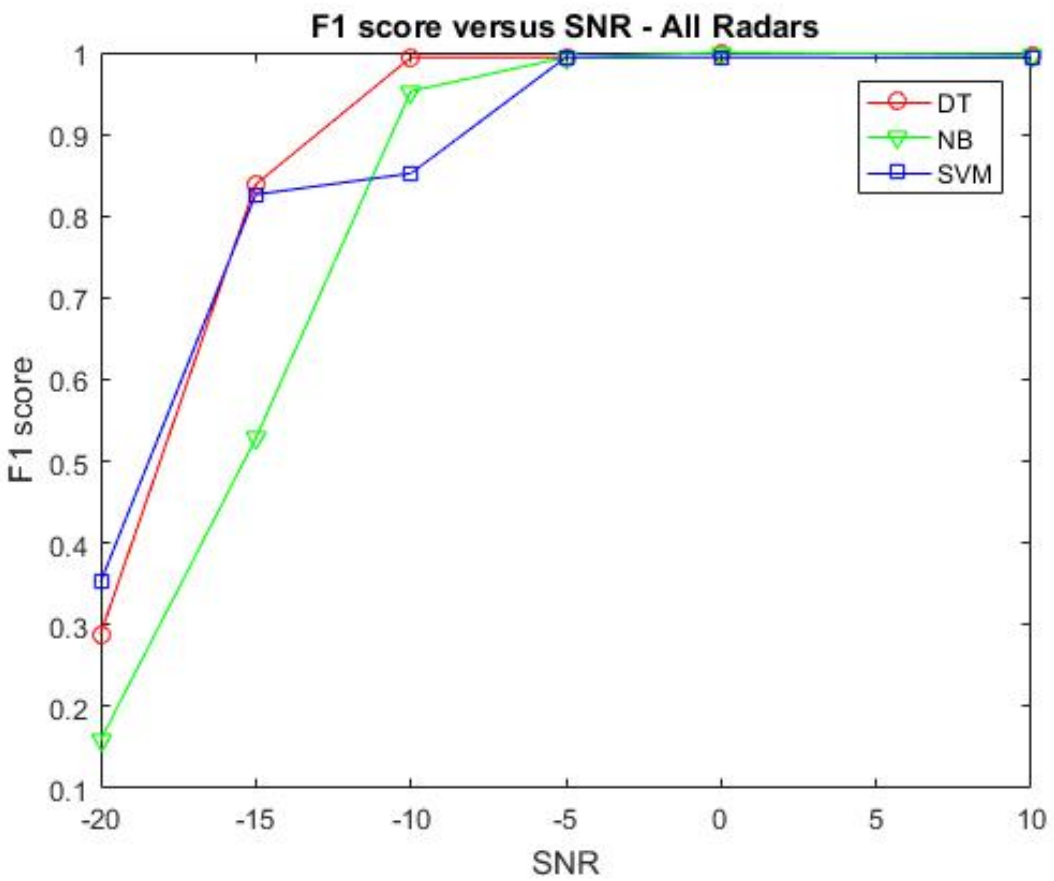

Figure 33: F1 score versus SNR level - All radar chirp rates. (Problem 5) 
portion of the pulse as illustrated in Figure 34.

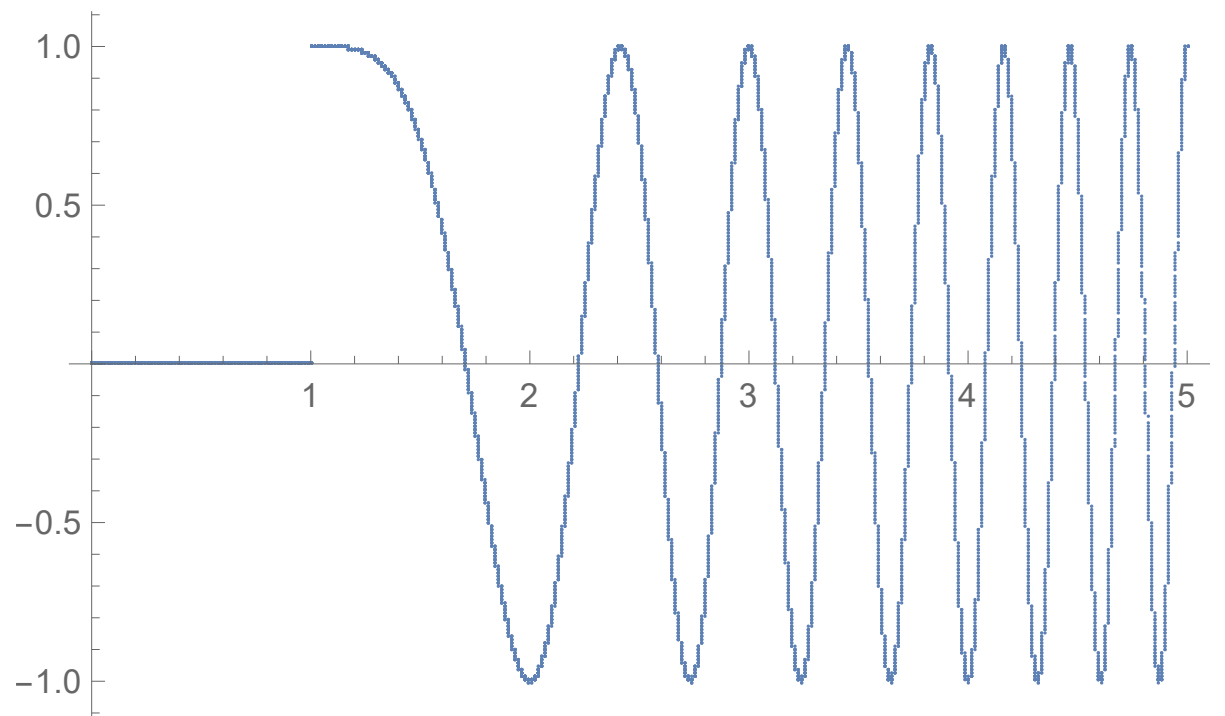

Figure 34: Truncated chirp of an LFM signal. (Problem 6)

For the proof-of-concept classifier, the chirp signal was truncated to determine if the classifier could detect and correctly classify the LFM signal when the pulse was detected at an unknown point in the pulse stream, as shown in Figure 34. For testing the robustness, $20 \%$ of the pulse was removed. The confusion matrix results of applying the NB, and SVM classifiers to a truncated chirp signal for all realistic radar modes are shown in Figures 35 and 36. The results for the DT classifier were inconclusive due to the simplicity of the classifier. Note that the classifiers were trained on the full pulse but tested with this truncated pulse.

All the four ML classifiers were applied but results only two best classifiers, NB and SVM are shown in this work. Both DT and RnF performed poorly at all SNRs.

Plots of accuracy and F1 scores at various SNR levels $(10 \mathrm{~dB}, 0 \mathrm{~dB},-5 \mathrm{~dB},-10$ $\mathrm{dB},-15 \mathrm{~dB}$ and $-20 \mathrm{~dB}$ ) are shown in Figure 37and Figure 38.

From the results, SVM slighlty outperforms NB at $0 \mathrm{~dB}$ but NB outperformed SVM at all other SNR levels. These results are significant as the algorithm indicates that we can classify $20 \%$ truncated pulse up to $0 \mathrm{~dB}$ with an accuracy and F1 score of 0.8. The results for the confusion matrices suggest that the SVM and Naive Bayes 

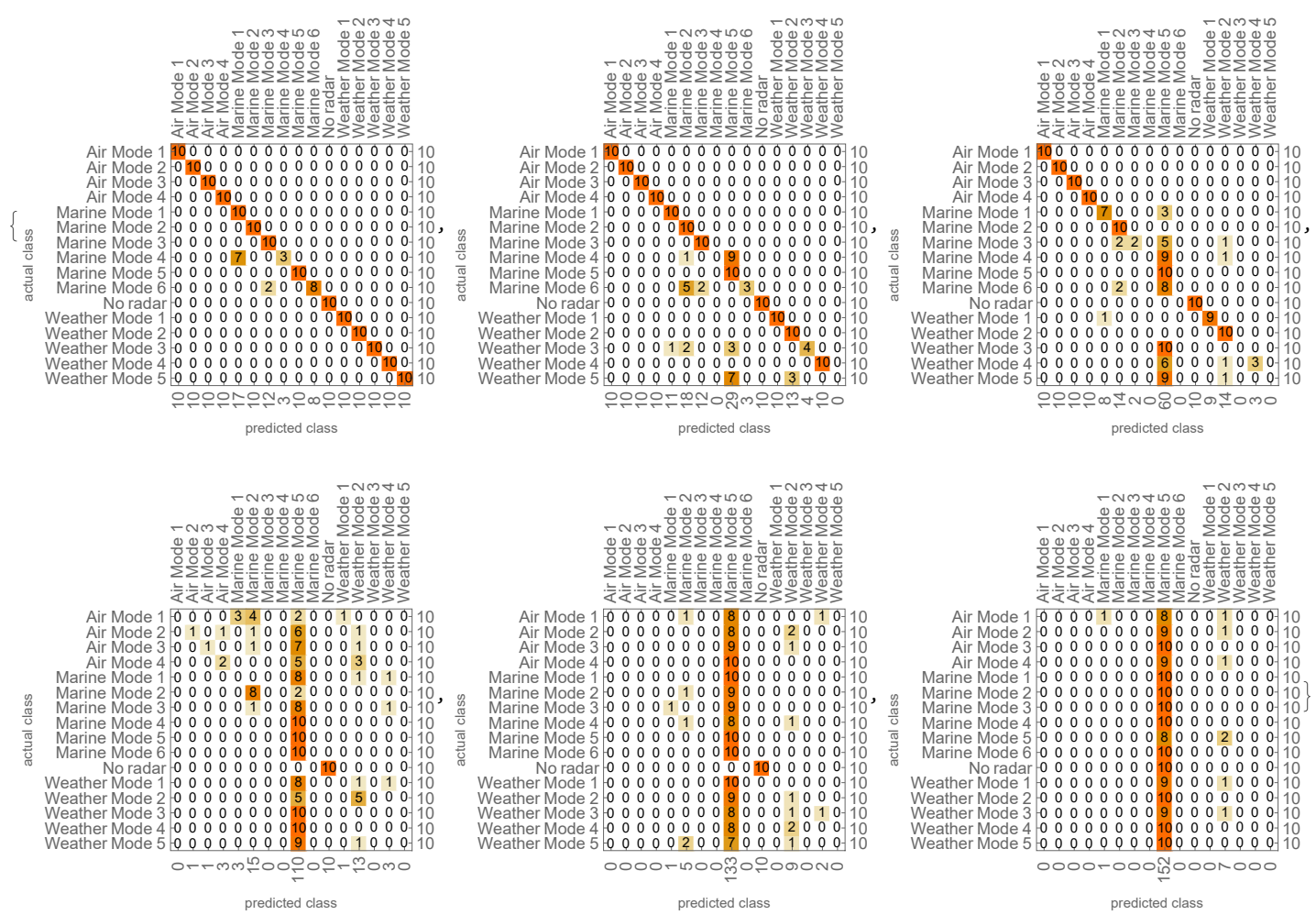

Figure 35: Confusion matrix for a NB classifier of realistic truncated chirp rates at SNR $10 \mathrm{~dB}$ (top left), $0 \mathrm{~dB}$ (top mid), $-5 \mathrm{~dB}$ (top right), -10 dB (bottom left), $-15 \mathrm{~dB}$ (bottom mid) and $-20 \mathrm{~dB}$ (bottom right). (Problem 6) 

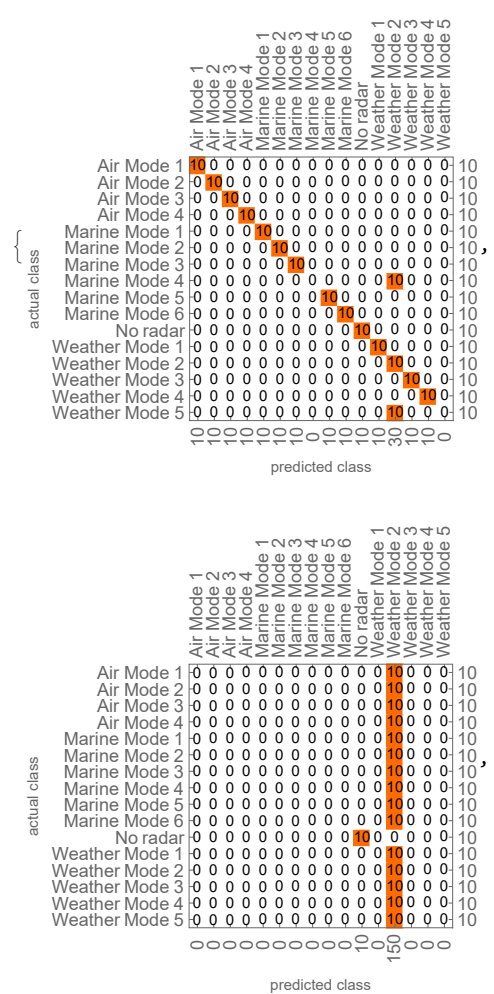
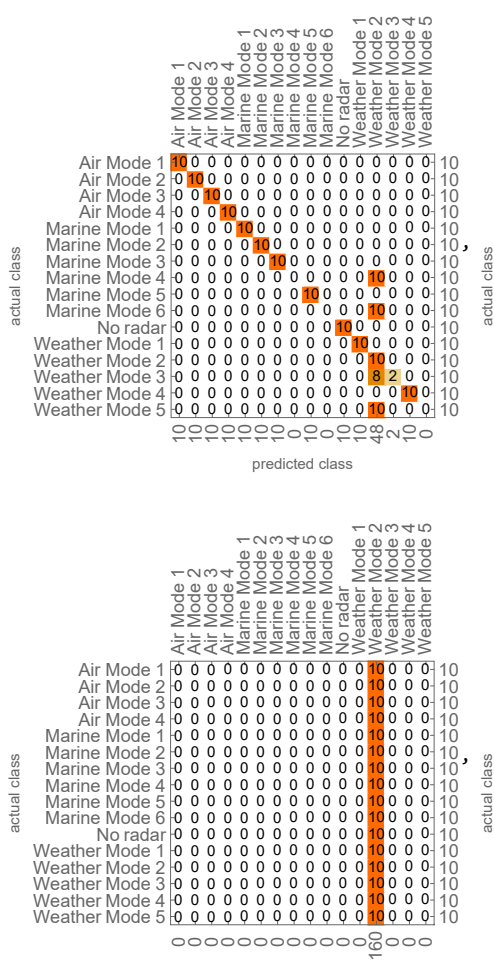

predicted class
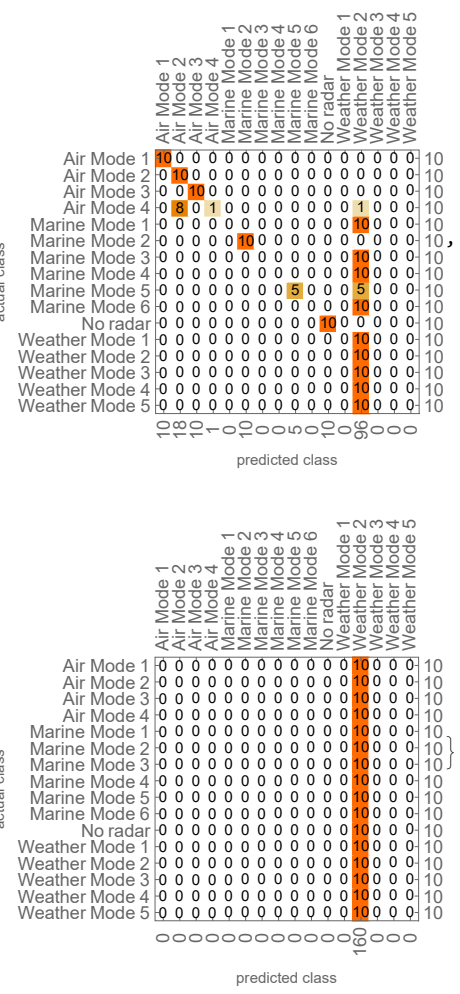

Figure 36: Confusion matrix for a SVM classifier of realistic chirp rates at SNR 10 $\mathrm{dB}$ (top left), $0 \mathrm{~dB}$ (top mid), $-5 \mathrm{~dB}$ (top right), $-10 \mathrm{~dB}$ (bottom left), $-15 \mathrm{~dB}$ (bottom mid) and $-20 \mathrm{~dB}$ (bottom right). (Problem 6) 


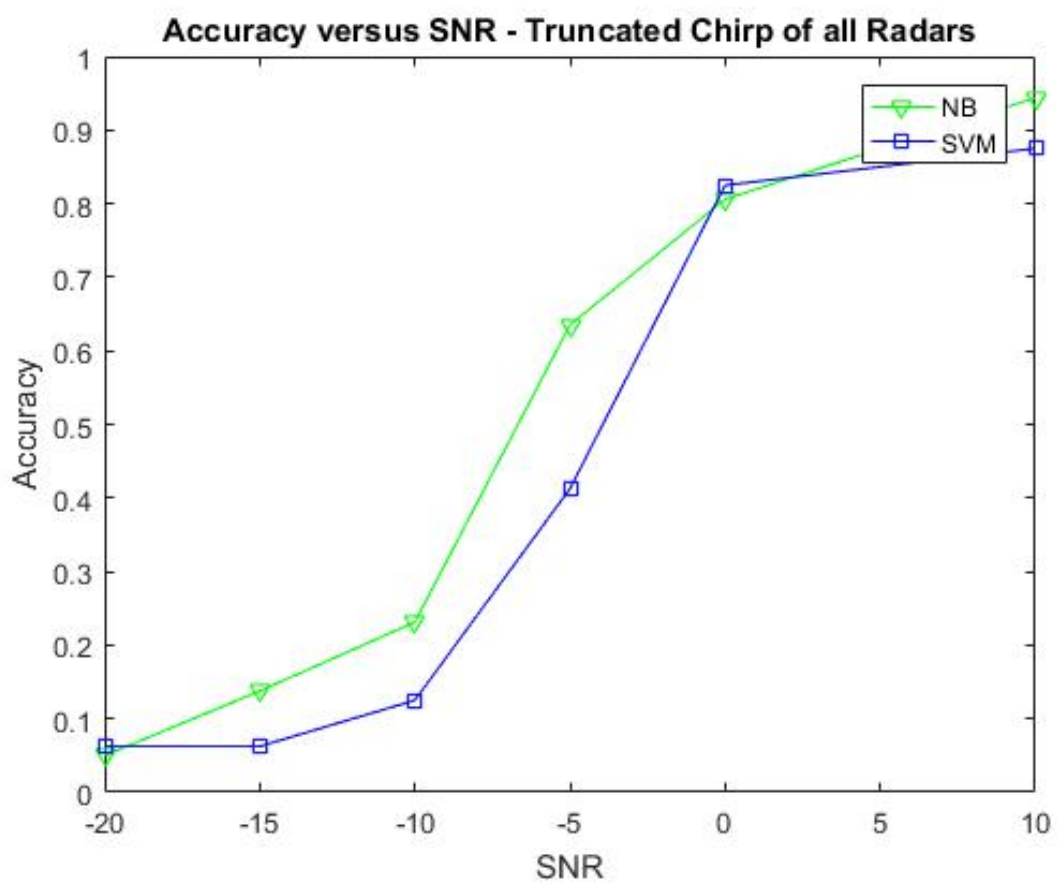

Figure 37: Accuracy versus SNR level - All radar truncated chirp rates. (Problem 6)

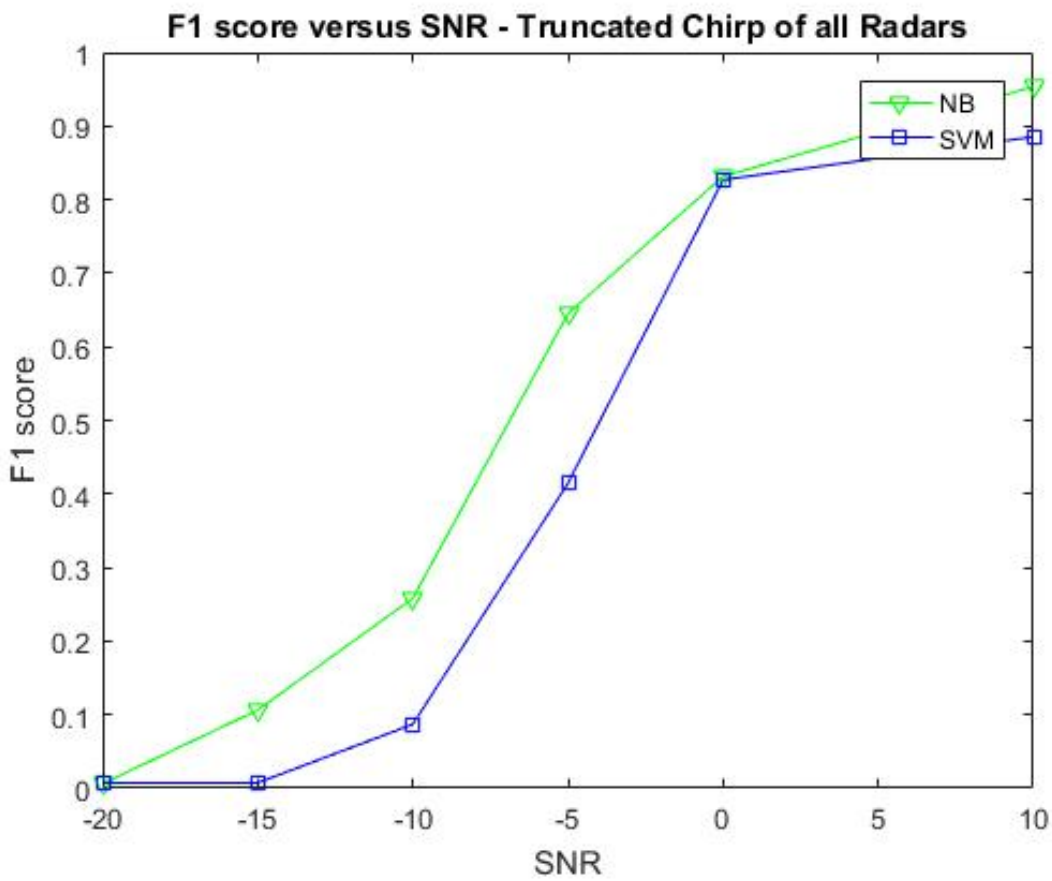

Figure 38: F1 score versus SNR level - All radar truncated chirp rates. (Problem 6) 
classifiers are tolerant to truncated pulses in some degree, as long as the SNR value is not too low.

\subsubsection{Problem 7: Robustness of the classifier against pertur- bation in chirp parameters.}

Since DT was the best performing classifier for Problem 5, the DT machine learning classifier was selected to determine if it could correctly determine the radar type based on the realistic chirp rates of Table 13. These radars are not nominally close to the realistic radar parameters. A tag was assigned to each unknown radar type to indicate which radar it should be similar to (radars with realistic parameters) in order to evaluate classifier performance.

The trained classifier from Problem 5 was tested using the simulated single LFM pulse using the parameters given in Table 13. Classification results are shown in Figures 39, 40, 41, 42, 43, and 44. Since the parameters are not close to the realistic parameters, the classifiers are not expected to perform perfectly even at high SNR. Interestingly, these simple classifiers are still able to provide reasonably good classification rates up to about $-10 \mathrm{~dB}$ SNR. Although the classifier does not always return the specific radar which is "most similar" to the one listed in the table, it selected another radar within the same class in most cases.

\subsection{Summary}

In this chapter, seven different classification problems were investigated. Examples were drawn from realistic parameters in airborne, marine and weather radars. Robustness against windowed received signal as well as parameter mismatch was also investigated. The general conclusion is that with the proposed concatenated match filtered output as the inputs to four well known machine learning algorithms give a reasonably good classification performance up to an SNR of -10 dB in almost all the cases studied in this thesis. 


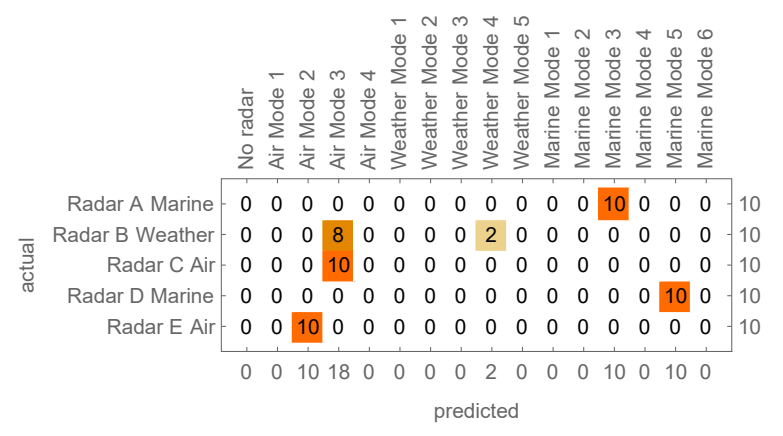

Figure 39: Confusion matrix for classifying signals from unknown radars at a SNR of $10 \mathrm{~dB}$ using a DT classifier. (Problem 7)

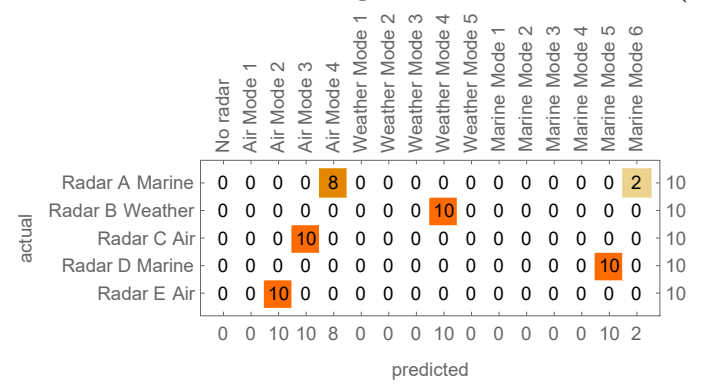

Figure 40: Confusion matrix for classifying signals from unknown radars at a SNR of $0 \mathrm{~dB}$ using a DT classifier. (Problem 7)

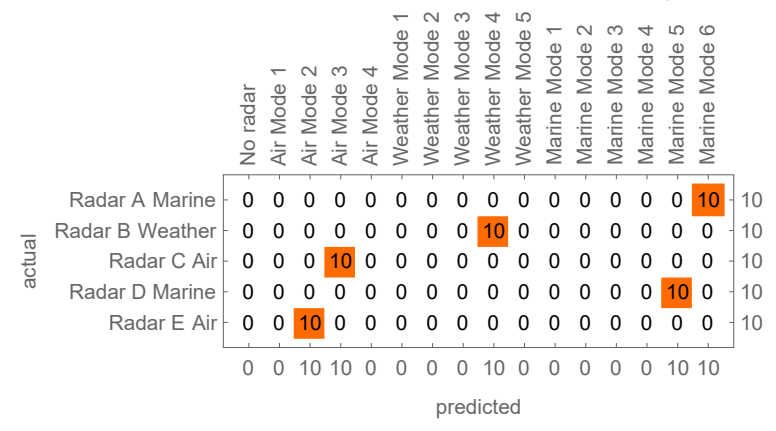

Figure 41: Confusion matrix for classifying signals from unknown radars at a SNR of $-5 \mathrm{~dB}$ using a DT classifier. (Problem 7) 


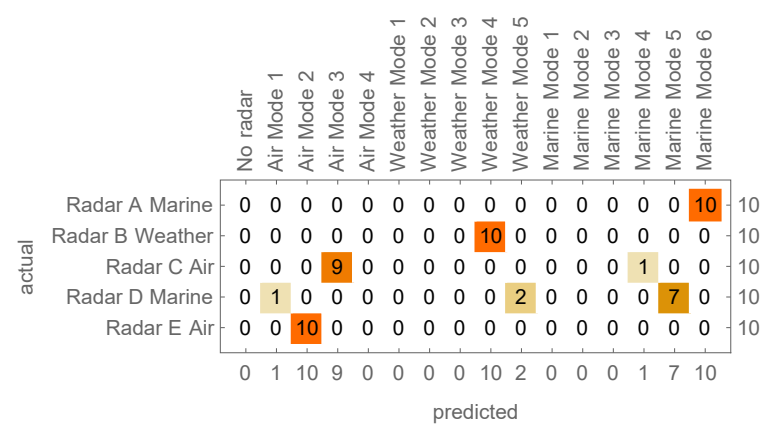

Figure 42: Confusion matrix for classifying signals from unknown radars at a SNR of $-10 \mathrm{~dB}$ using a DT classifier. (Problem 7)

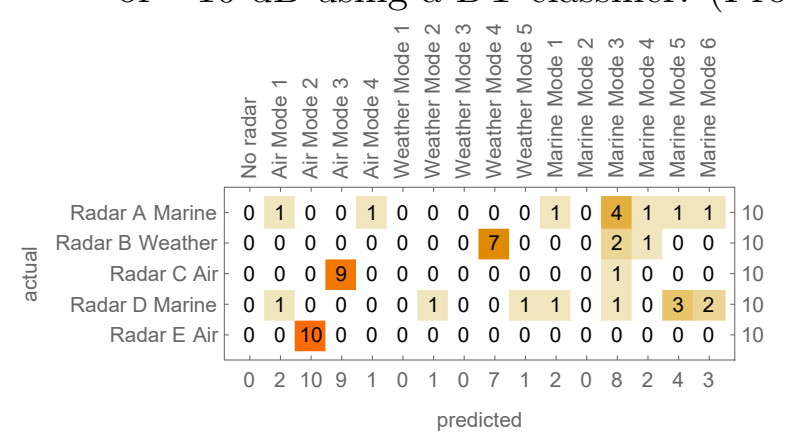

Figure 43: Confusion matrix for classifying signals from unknown radars at a SNR of $-15 \mathrm{~dB}$ using a DT classifier. (Problem 7)

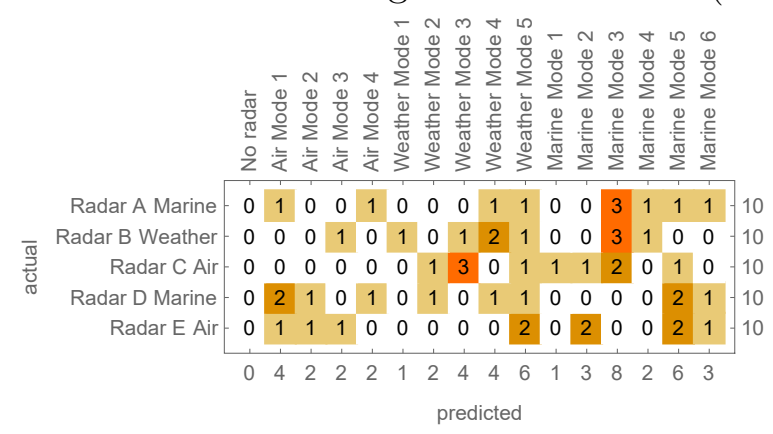

Figure 44: Confusion matrix for classifying signals from unknown radars at a SNR of $-20 \mathrm{~dB}$ using a DT classifier. (Problem 7) 


\section{Chapter 6}

\section{Conclusions and Way Forward}

This thesis has proposed an approach to identify the unknown radar chirp rate, $\beta$, from a known set of chirp rates, of an incoming LFM radar signals at SNR values as low as -20 dB. Supervised machine learning algorithms have been selected to identify pulsed LFM radars using the outputs of matched filtering. The approach has been validated with simulations. The results presented in this thesis represent only a preliminary application of the combination of matched filtering and machine learning. No feature extraction is attempted in this thesis. A concatenated matched filter outputs from the matched filters corresponding to the known set of chirp rates were presented to the classifiers used in this thesis.

The approach was tested on simulated LFM signals with pre-selected chirp rates. The method has the drawback that it requires the set of possible LFM parameters to be known in advance. However, this is not an unreasonable assumption in the context of spectrum monitoring. This approach can be used to determine the presence or absence of known radar emitters, or to show the operational or non-operational state of these emitters potentially even in a low-SNR scenario. Low-SNR scenarios can occur if the receiver is situated along the side-lobes of the transmitting radar antenna.

Four types of machine learning classifiers namely, the decision tree, the naive Bayes, the random forest and the support vector machines are used in this thesis. Three types of radars namely airborne, marine and weather radars are considered in this research work along with their modes. Airborne radar has 4 modes, weather radar has 5 modes while marine radar has 6 modes. Classifiers for each type of radar is designed and the modes of the radars are identified with single LFM pulses received from the same type of radar. Thus in the case of classifiers for airborne, weather 
and marine radars, the classifier output has 5, 6 and 7 classes. The extra class in each of these radars corrected to noise class (same as "no radar" class). Thus, the input features to each of the above classifiers are outputs of a bank of 5, 6 and 7 matched filters. Another 16-way classification is also considered where the classifiers take the output of a bank of 16 matched filters is also trained to classify the radar type along with its mode. This three 16-way classifiers based on DT, NB and SVM are considered. In order to study the robustness of these 16-way classifiers, a set of 5 radars whose chirp parameter is close but not exactly matching any of the parameters in the known set of chirp parameters is considered. As the modes do not match, this set of 5 radars is considered similar to certain radars and their types. Test data consisting of single pulses from these 5 radars is presented to the classifier and the output of the classifier is analyzed. As one cannot expect the classifier to correctly identify the mode, if the classifier declares the class corresponding to the radar which the inputs are similar to, then the classifier is considered to be robust. Furthermore, the 16-way classifier is checked for its classification robustness to imperfect pulse. Instead of having a full pulse, only $80 \%$ of the pulse is presented and the ability of the classifier to still output a correct class is verified.

The proof-of-concept toy problem indicated that simple machine learning classifiers trained using concatenated matched filter outputs were able to classify noisy single pule LFM radar signals.

For classification systems trained with airborne radar single LFM pulses, DT performed the best, and provided an acceptable performance even at $-20 \mathrm{~dB}$ when the classifier was presented with noisy single LFM pulses from airbone radars. DT and SVM gave nearly identical performance for weather radars, and very good performance until $-15 \mathrm{~dB}$ For systems trained with marine radar, DT gave the best performance, with very good performance down to about $-10 \mathrm{~dB}$.

In a 16-class problem, DT outperformed other classifiers when there a perfect match in the parameters and full single pulse LFM signals were assumed at the receiver end.

Surprisingly DT did not perform well for the truncated chirp. NB and SVM performed well until an SNR of $0 \mathrm{~dB}$, when the truncation was at 20\%. Since the study was done under noisy conditions, any more truncation would lead to serious under performance and that was not investigated. The simulation results suggest that the NB and SVM classifiers are more tolerant to imperfect pulses than DT and 
RnF.

\subsection{Limitations}

This investigations carried out in the thesis have the following limitations:

- The radar waveform is assumed to be LFM.

- The chirp rate of the LFM signal is a member of a known set of chirp rates.

- The signal is assumed to be adequately sampled.

- The received signal is only assumed to be corrupted by additive noise.

- Only simple machine learning algorithms were considered. For instance, neural networks were not considered.

- Experiments for real data collection could not be carried out due to the pandemic situation. Therefore, this study is purely done based on simulations and may not reflect accurately the real world performance.

- Only one emitter was considered to be present in the simulations.

- It is assumed in this study that the radar pulse has been correctly de-interleaved.

\subsection{Future work}

Some possible areas to consider in future research are as follows:

- Assessment of performance with real radar LFM signals.

- Superposition of multiple LFM signals which normally occur in a RF congested environment.

- Nonlinear FM signals such as those defined in [76], [77].

- Optimization of the SVM parameters by hyperparameter tuning.

- Classification using shallow and deep neural networks.

- Assessment of performance with sophisticated radar waveforms such as ultrawide band noise radars. 


\section{List of References}

[1] ITU, "Technical characteristics of maritime radio navigation radars," Tech. Rep. ITU-R.M.1313, International Maritime Organization, 2018.

[2] Radar Spectrum Engineering and Management: Technical and Regulatory Issues, 2015.

[3] P. E. Pace, Detecting and Classifying Low Probability of Intercept Radars. Artech House, 2004.

[4] L. Stankovic, M. Dakovic, and T. Thayparan, Time-Frequency Signal Analysis with Applications. Artech House, 2002.

[5] X. Liu, T. Li, X. Fan, and Z. Chen, "Nyquist zone index and chirp rate estimation of LFM signal intercepted by Nyquist folding receiver based on random sample consensus and Fractional Fourier Transform," Sensors, vol. 19, no. 6, pp. 14771491, 2019.

[6] J. Song, X. Xu, and G. Li, "An accurate parameter estimator for LFM signals based on zoom modified discrete chirp Fourier transform," IAENG International Journal of Computer Science, vol. 46, no. 3, pp. 78-85, 2019.

[7] J. Zheng, H. Liu, and Q. H. Liu, "Parameterized centroid frequency-chirp rate distribution for LFM signal analysis and mechanisms of constant delay introduction," IEEE Transactions on Signal Processing, vol. 65, pp. 6435-6447, Dec. 2017.

[8] D. Djurovic, C. Ioana, L. Stankovic, and P. Wang, "Adaptive algorithm for chirprate estimation," EURASIP Journal on Advances in Signal Processing, 2009.

[9] D. Zeng, H. Xiong, K. Long, and B. Tang, "A fast recognition algorithm for three kinds of intra-pulse modulation signals," in 2009 IET International Radar Conference, pp. 1-4, Apr. 2009.

[10] M. Wu, X. Dai, Y. D. Zhang, B. Davidson, M. G. Amin, and J. Zhang, "Fall detection based on sequential modeling of radar signal time-frequency features," in IEEE International Conference on Healthcare Informatics, pp. 169-174, Sept. 2013.

[11] S. Haykin and T. Bhattacharya, "Wigner-Ville distribution: An important functional block for radar target detection in clutter," in Proceedings of 1994 28th Asilomar Conference on Signals, Systems and Computers, vol. 1, pp. 68-72, 1994. 
[12] P. K. Kumar and K. M. M. Prabhu, "Classification of radar returns using WignerVille distribution," in 1996 IEEE International Conference on Acoustics, Speech, and Signal Processing Conference Proceedings, vol. 6, pp. 3105-3108, May 1996.

[13] S. Sirianunpiboon, "Extended array method for detection and direction finding of a weak linear frequency modulated signal," in 2011 IEEE International Conference on Acoustics, Speech and Signal Processing (ICASSP), pp. 2560-2563, May 2011.

[14] A. Ahmad, A. Muhammad Lawan, M. Ajiya, and Z. Yusuf, "Sorting of low probability of intercept radar signals based on frequency modulation constancy using Wigner-Ville distribution," in 2019 IEEE Asia-Pacific Conference on Applied Electromagnetics (APACE), pp. 1-5, Nov. 2019.

[15] L. Zhang, X. He, Y. Li, M. Xing, and Z. Bao, "Parameters estimation of LFM signals based on STTFD," in ICSP 2008 Proceedings, 2008.

[16] T. O. Gulum, A. Y. Erdogan, T. Yildirim, and P. E. Pace, "A parameter extraction technique for FMCW radar signals using Wigner-Hough-Radon transform," in 2012 IEEE Radar Conference, pp. 0847-0852, May 2012.

[17] T. O. Gulum, A. Y. Erdogan, T. Yildirim, and L. Durak Ata, "Parameter extraction of FMCW modulated radar signals using Wigner-Hough transform," in 2011 IEEE 12th International Symposium on Computational Intelligence and Informatics (CINTI), pp. 465-468, Nov. 2011.

[18] K. K. Guner, B. Erkmen, T. O. Gulum, A. Y. Erdogan, T. Yıldırım, and L. Durak Ata, "Implementation aspects of Wigner-Hough transform based detectors for LFMCW signals," in 2016 39th International Conference on Telecommunications and Signal Processing (TSP), pp. 441-444, June 2016.

[19] F. G. Geroleo, M. Brandt-Pearce, and C. L. Brown, "Detection and estimation of multi-pulse LFMCW radar signals," in 2010 IEEE Radar Conference, pp. 10091013, May 2010.

[20] W. Niczyporuk, "LFM radar signal detection in the joint time-frequency domain," in Signal Processing Algorithms, Architectures, Arrangements, and Applications SPA 200\%, pp. 33-37, Sept. 2007.

[21] A. Bouchikhi, A. Boudraa, J. Cexus, and T. Chonavel, "Analysis of multicomponent LFM signals by Teager-Huang-Hough transform," IEEE Transactions on Aerospace and Electronic Systems, vol. 50, pp. 1222-1233, April 2014.

[22] S. Sirianunpiboon, S. D. Howard, and S. D. Elton, "A group invariance approach to a very weak LFM signal detection," in 2018 IEEE Statistical Signal Processing Workshop (SSP), pp. 698-701, June 2018.

[23] E. Cheng, S. Chen, and F. Yuan, "Design and detection of multilinear chirp signals for underwater acoustics sensor networks," International Journal of Distributed Sensor Networks, vol. 11, Nov. 2015. 
[24] A. Serbes, "A method for estimating chirp rate of a LFM signal," Balkan Journal of Electrical and Computer Engineering, vol. 6, pp. 9-12, Feb. 2018.

[25] A. Serbes and O. Aldimashki, "A fast and accurate chirp rate estimation algorithm based on FrFT," in 2017 25th European Signal processing Conference, 2017.

[26] O. Aldimashki and A. Serbes, "Performance of chirp parameter estimation in the fractional fourier domains and an algorithm for fast chirp-rate estimation," IEEE Transactions on Aerospace and Electronic Systems, vol. 56, pp. 3865-3700, Oct. 2020.

[27] Z. Qiu, J. Zhu, and F. Li, "Multiple BPSK/LFM hybrid modulated signals parameter estimation and analysis intercepted by non-cooperative radar receiver," in 2018 IEEE International Conference on Computational Electromagnetics (ICCEM), pp. 1-4, Mar. 2018.

[28] Y. Ma, R. Wang, and J. Du, "Amplitude characteristics of linear frequency modulation signal in FRFT domain," in 2013 IEEE International Conference on Signal and Image Processing Applications, pp. 431-434, Oct. 2013.

[29] Q. Chen, Y. Li, and M. Zhu, "Fast algorithm for parameter estimation of LFM signals under low SNR," in AIP Conference Proceedings, vol. 1839, 2017.

[30] D. Fourer, F. Auger, K. Czarnecki, S. Meignen, and P. Flandrin, "Chirp rate and instantaneous frequency estimation:application to recursive vertical synchrosqueezing," IEEE Signal Processing Letters, vol. 24, pp. 1724-1728, Nov. 2017.

[31] G. Turin, "An introduction to matched filters," IRE Transactions on Information Theory, vol. 6, pp. 311-329, June 1960.

[32] S. Salem, "Enhancement of matched filter response for chirp radar signals using signal recovery method," International Journal of Innovative Technology and Exploring Engineering, vol. 9, no. 2, pp. 561-565, 2019.

[33] A. Selim, F. Paisana, J. Arokkiam, Y. Zhang, and L. Doyle, "Spectrum monitoring for radar bands using deep convolutional neural networks," in 2017 IEEE Global Communications Conference, pp. 1-6, Dec. 2017.

[34] M. Kong, J. Zhang, W. Liu, and G. Zhang, "Radar emitter identification based on deep convolutional neural network," in International Conference on Control, Automation and Information Sciences, pp. 309-314, Oct. 2018.

[35] L. Wang, H. Ma, Q. Li, and Z. Li, "Multicomponent linear FM signal detection based on support vector clustering," in 5th International Conference on Information Communications Signal Processing, pp. 743-747, 2005.

[36] P. Ruiz, J. Mateos, G. Camps-Valls, R. Molina, and A. K. Katsaggelos, "Bayesian active remote sensing image classification," IEEE Transactions on Geoscience and Remote Sensing, vol. 52, pp. 2186-2196, Apr. 2014. 
[37] J. Lunden, L. Terho, and V. Koivunen, "Waveform recognition in pulse compression radar systems," in 2005 IEEE Workshop on Machine Learning for Signal Processing, pp. 271-276, 2005.

[38] E. Fischell and H. Schmidt, "Supervised machine learning for estimation of target aspect angle from bistatic acoustic scattering," IEEE Journal of Oceanic Engineering, pp. 759-769, Oct. 2017.

[39] J. Seok, "Active sonar target classification using classifier ensembles," International Journal of Engineering Research and Technology, vol. 11, no. 12, pp. 21252133, 2018.

[40] S. El-Khamy and H. Elsayed, "Classification of multi-user chirp modulation signals using wavelet higher-order statistics features and artificial intelligence techniques," International Journal of Communications, Network and System Sciences, vol. 5, pp. 520-533, Sept. 2012.

[41] B. Boashash and S. Ouelha, "Automatic signal abnormalilty detection using time-frequency features and machine learning: A newborn EEG seizure study," Knowledge-based Systems, vol. 106, pp. 38-50, Aug. 2016.

[42] H. Su, Q. Bao, and Z. Chen, "Parameter estimation processor for chirp signals based on a complex-valued deep neural network," IEEE Access, vol. 7, pp. 176278-176290, 2019.

[43] G. Lee, J. Jo, and C. Park, "Jamming prediction for radar signals using machine learning methods," Security and Communications Networks, vol. 2020, 2020.

[44] L. Gao, X. Zhang, J. Gao, and S. You, "Fusion image-based radar signal feature extraction and modulation recognition," IEEE Access, vol. 7, pp. 13135-13148, 2019.

[45] F. Wang, S. Huang, H. Wang, and C. Yang, "Automatic modulation classification exploiting hybrid machine learning network," Mathematical Problems in Engineering, vol. 2018, p. 14, 2018.

[46] G. Noone, "Radar pulse classification using support vector machine," in Workshop on the Application of radio Science National Committee for Radio Science, 2000 .

[47] J. Kwon, S. Lee, and N. Kwak, "Radar application of deep neural networks for recognizing micro-Doppler radar signals by human walking and background noise," in 2018 International Symposium on Antennas and Propagation (ISAP), pp. 1-2, 2018.

[48] S. Hong, Y. Yi, J. Jo, and B. Seo, "Classification of radar signals with convolutional neural networks," in 2018 Tenth International Conference on Ubiquitous and Future Networks (ICUFN), pp. 894-896, 2018.

[49] M. V. i. Carretero, R. I. A. Harmanny, and R. P. Trommel, "Smart-CFAR, a machine learning approach to floating level detection in radar," in 2019 16th European Radar Conference (EuRAD), pp. 161-164, 2019. 
[50] M. Ren, J. Cai, Y. Zhu, and M. He, "Radar emitter signal classification based on mutual information and fuzzy support vector machines," in ICSP2008 Proceedings, pp. 1641-1646, 2008.

[51] J. M. Kurdzo, J. Y. N. Cho, B. L. Cheong, and R. D. Palmer, "A neural network approach for waveform generation and selection with multi-mission radar," in 2019 IEEE Radar Conference (RadarConf), pp. 1-6, 2019.

[52] O. Mughal and S. Kim, "Signal classification and jamming detection in wideband radios using Naive Bayes classifier," IEEE Communications Letters, vol. 22, pp. 1398-1401, July 2018.

[53] D. Xu, L. Du, H. Liu, and D. Luo, "Sparse bayesian multitask learning for radar target recognition," in 2016 CIE International Conference on Radar (RADAR), pp. 1-4, 2016.

[54] L. Wang, H. Ma, Q. Li, and Z. Li, "Multicomponent LFM signal detecetion based on support vector clustering," in 5th International Conference on Information Systems and Signal Processing, pp. 743-747, 2005.

[55] R. Caroni and M. Souryal, "Detection of incumbent radar in the $3.5 \mathrm{GHz}$ CBRS band," in 2018 IEEE Global Conference on Signal and Information Processing (GlobalSIP), pp. 241-245, 2018.

[56] M. Skolnik, Introduction to Radar Systems. McGraw-Hill, 2 ed., 1980.

[57] M. Tipping, "The relevance vector machine," in Advances in Neural Information Processing Systems, pp. 652-658, 2000.

[58] D. Anguita, A. Ghio, N. Greco, L. Oneto, and S. Ridella, "Model selection for support vector machines:advantages and disadvantages of the machine learning theory," in The 2010 International Joint Conference on Neural Networks (IJCNN), pp. 1-8, 2010.

[59] S. Archana and K. Elangovan, "Survey of classification techniques in data mining," International Journal of Computer Science and Mobile Applications, vol. 1, pp. 268-278, Nov. 2014.

[60] S. L. Crawford, "Extensions to the CART algorithm," International Journal of Man-Machine Studies, vol. 31, no. 2, pp. 197-217, 1989.

[61] T. Buskirk, "Surveying the forests and sampling the trees: An overview of classification and regression trees and random forests with applications in survey research," Survey Practice, vol. 11, no. 1, 2018.

[62] R. Kohavi and F. Provost, "Guest editors' introduction: On applied research in machine learning," Machine Learning, vol. 30, pp. 127-132, Feb. 1998.

[63] A. Ragan, "Taking the confusion out of confusion matrices," www.towardsdatascience.com, Oct. 2018. Accessed April 2020. 
[64] M. Kubat, R. C. Holte, and S. Matwin, "Machine learning for the detection of oil spills in satellite radar images," in Machine Learning, vol. 30, pp. 195-215, 1998.

[65] F. Giannini, K. Lupinetti, and M. Monti, "Identification of similar and complementary subparts in B-Rep mechanical models," Journal of Computers and Information Science, vol. 17, Dec. 2017.

[66] D. S. Zrnic and R. J. Doviak, "System requirements for phased array weather radar," tech. rep., National Severe Storms Laboratory, 2018.

[67] M. Gabella, R. Notarpietro, S. Bertoldo, A. Prato, C. Lucianaz, O. Rorato, M. Allegretti, and G. Perona, Doppler Radar Observations: Weather Radar, Wind Profiler, Ionospheric Radar, and Other Advanced Applications, ch. "A network of portable, low-cost X-band radars, Doppler radar Observations, network of portable, low-cost radars". IntechOpen, 2012.

[68] "Operators manual WR-2100, https://fccid.jp/ad89zwwr2100/usermanual/user-manual 2470823, (accessed june 29, 2018)."

[69] M. Wada, N. Anraku, H. Yamauchi, and A. Adachi, "Operational usage of solidstate weather radar," in WMO technical conference on meteorological and environmental instruments and methods of observation (TECO), 2012.

[70] J. L. Salazar, E. A. Knapp, and D. J. McLuaghlib, "Antenna design trade-offs for a dense, distributed radar network for weather sensing," in Proc. 33 International Conference on Radar Meteorology, 2007.

[71] N. A. Press, "Weather radar technology beyond NEXRAD," tech. rep., Committee on Weather Radar Technology Beyond NEXRAD, 1982.

[72] A. Nelander and Z. Tóth-Pál, "Modular system design for a new S-band marine radar," in International Radar Conference, pp. 1-5, 2009.

[73] Furono Operator's Manual - Model 7111, 1994.

[74] M. E. Weber, "FAA surveillance radar data as a complement to the WSR-88D network," tech. rep., https://www.cv.nrao.edu, 2005.

[75] C. Lesnik, "Nonlinear frequency modulated signal design," Acta Physica Polonica A, vol. 116, no. 3, pp. 351-354, 2009.

[76] A. W. Doerry, "Generating nonlinear FM chirp waveforms for radar," tech. rep., Sandia Report SAND2006-5856, 2006.

[77] K. J. Abratkiewicz and P. Samczytiski, "A block method using the chirp rate estimation for NLFM radar pulse reconstruction," Sensors, vol. 19, no. 22, p. 5015, 2019. 